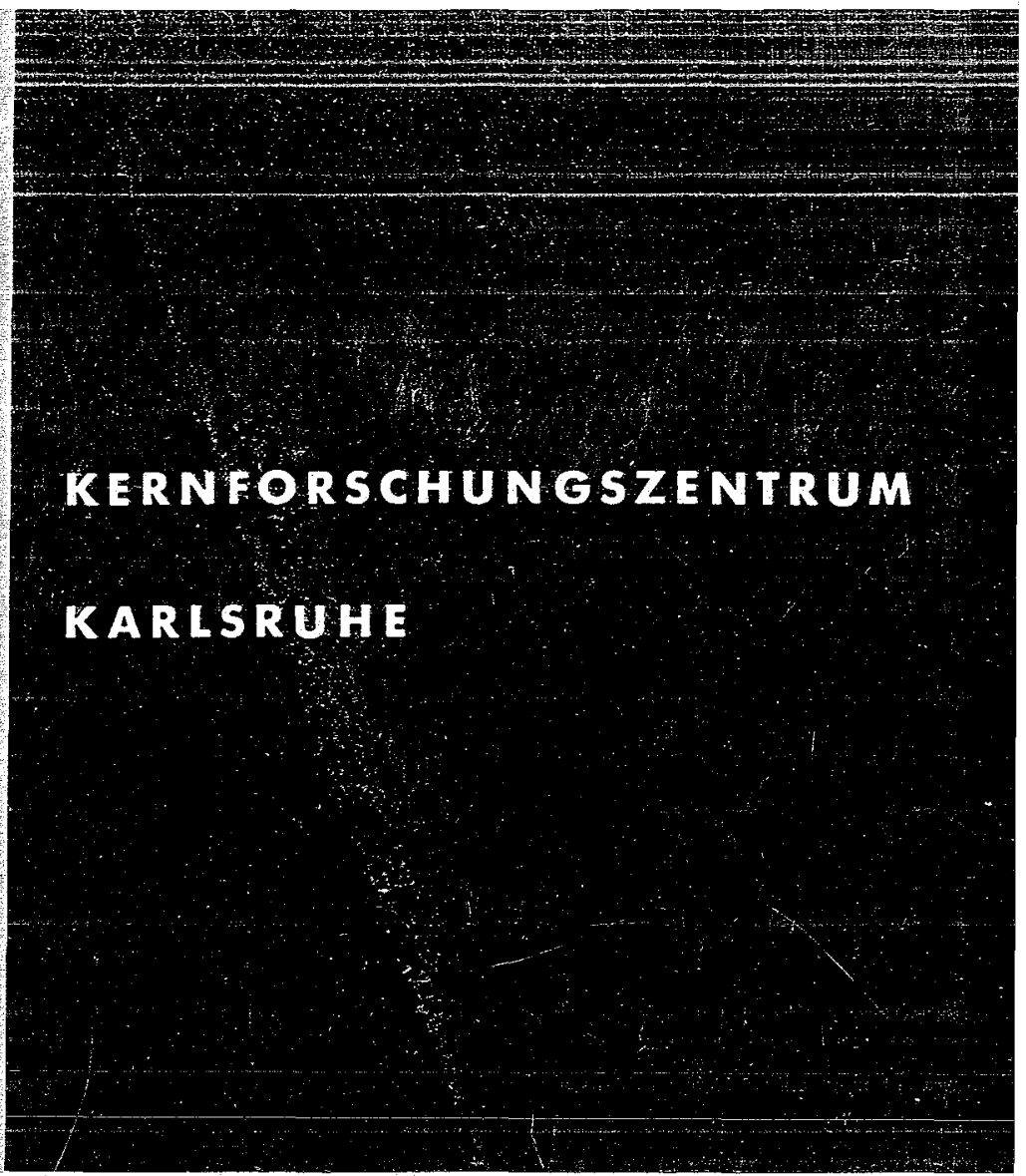

Institut für Feiße Chemie

Die Wiederaufarbeitung von Uran-Plutonium-Kernbrennstoffen

\title{
F. Baumgartner
}

unter Mitwirkung von $\mathrm{H}$. Philipp

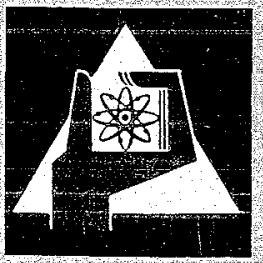

GESELLSCHATT FUR KERNFORSCHUNG M.B.M. KAR LSR UHE 



\title{
Fortschritse der chemischen Forschung Sonderdruck aus Band 12, Heft 4
}
Herausgeber: A. Davison, M. J. S. Dewar, K. Hafner, E. Heilbromner,
$U$. Hofmann, K. Niedenzu, Kl. Schäfer, G. Wittig
Scbriftleitung: $F$. Boscbke

\author{
Springer-Verlag Berlin Heidelberg New York 1969 \\ Printed in Germany - Nicht im Handel
}

F. Baumgärtner und $H$. Philipp

Die Wiederaufarbeitung

von Uran-Plutonium-Kernbrennstoffen 

Die Wiederaufarbeitung von

Uran-Plutonium-Kernbrennstoffen

\section{Prof. Dr. F. Baumgärtner}

Kernforschungszentrum Karlsruhe, Lehrstuhl für Radiochemie der Universität Heidelberg

\section{unter Mitwirkung von Dr. H. Philipp}

Literaturabteilung des Kernforschungszentrums Karlsruhe 


\section{Inhalt}

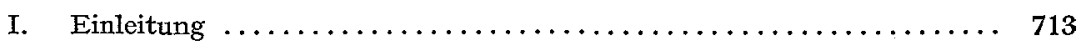

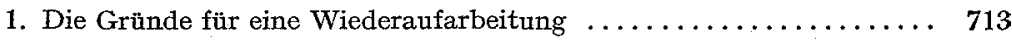

2. Der nukleare Abbrand und die Plutonium-Erzeugung ......... 714

3. Der Unterschied ziviler und militärischer Wiederaufarbeitung ..... 716

a) Brennstoffumhüllung und Aufschlußverfahren $\ldots \ldots \ldots \ldots \ldots, 716$

b) Spaltproduktkonzentration und Strahlung $\ldots \ldots \ldots \ldots \ldots \ldots \ldots, 717$

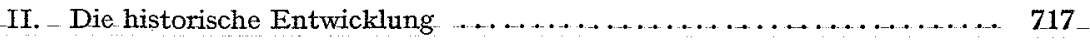

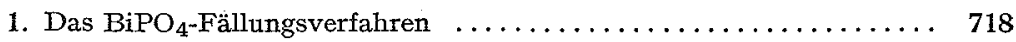

2. Die Extraktionsverfahren $\ldots \ldots \ldots \ldots \ldots \ldots \ldots \ldots \ldots \ldots \ldots$

a) Die Hexon-Verfahren $\ldots \ldots \ldots \ldots \ldots \ldots \ldots \ldots \ldots \ldots \ldots \ldots \ldots \ldots, 720$

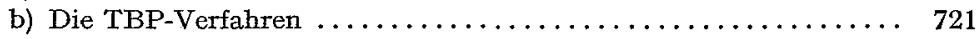

c) Andere Extraktionsverfahren $\ldots \ldots \ldots \ldots \ldots \ldots \ldots \ldots \ldots, 723$

III. Die Chemie des Purex-Verfahrens . ................... 726

1. Der Extraktionsvorgang bei Uran, Plutonium, $\mathrm{HNO}_{3}$ und einigen

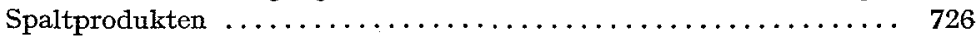

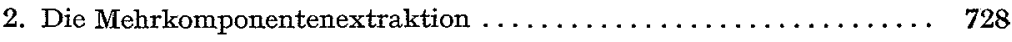

3. Die extraktive Uran-Plutonium-Trennung $\ldots \ldots \ldots \ldots \ldots \ldots \ldots .729$

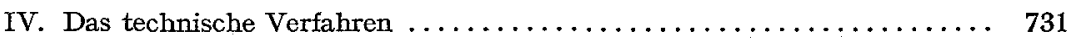

1. Die Anordnung der Cyclen $\ldots \ldots \ldots \ldots \ldots \ldots \ldots \ldots \ldots \ldots \ldots, 735$

2. Das Verfahren mit erhöhter Temperatur $\ldots \ldots \ldots \ldots \ldots \ldots \ldots \ldots, 736$

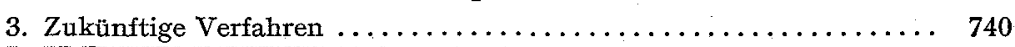

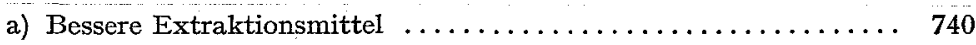

b) Unkonventionelle Prozesse und das „Aqua-Fluor-Verfahren“ ... 741

V. Entwicklungsprobleme der wäßrigen Wiederaufarbeitung $\ldots \ldots \ldots \ldots 742$

1. Die chemische Form des Brennstoffs bei zukünftigen Uran-Plutonium-

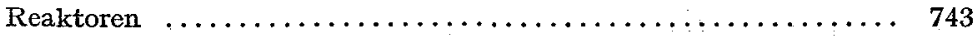

a) Der erhöhte Plutonium-Gehalt $\ldots \ldots \ldots \ldots \ldots \ldots \ldots \ldots \ldots \ldots \ldots$

b) Carbidische Brennstoffe $\ldots \ldots \ldots \ldots \ldots \ldots \ldots \ldots \ldots \ldots \ldots \ldots \ldots \ldots \ldots \ldots$ 
2. Abbrand, spezifische Stableistung und Kühlzeit zukünftiger Brennstäbe 747

a) Nachwärmeleistung ........................ 748

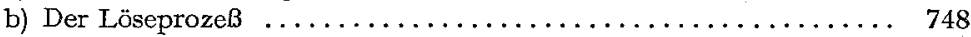

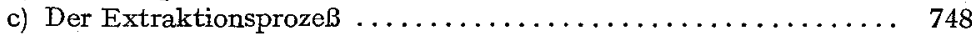

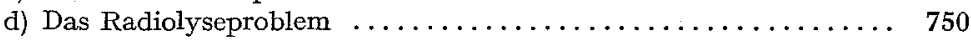

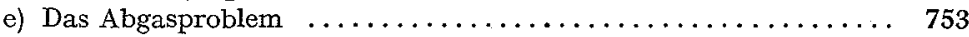

VI. Gewinnung von Batterie-Nucliden $\ldots \ldots \ldots \ldots \ldots \ldots \ldots \ldots \ldots \ldots . \ldots \ldots 9$

1. Die Batterie-Nuclide und ihre Bildung in Reaktoren . . . . . . . . 759

2. Die Gewinnung von Neptunium-237 ................. 762

3. Americium und Curium aus dem hochaktiven Abfall ......... 762

VII. Literatur $\ldots \ldots \ldots \ldots \ldots \ldots \ldots \ldots \ldots \ldots \ldots \ldots \ldots \ldots \ldots \ldots \ldots$

\section{Einleitung}

\section{Die Gründe für eine Wiederaufarbeitung}

Drei Argumente sprechen für eine chemische Wiederaufarbeitung verbrauchter Brennstäbe von Kernreaktoren. Der wichtigste Grund ist die Rückgewinnung von spaltbarem Material. Ein großer Teil der zivilen Kernkraftwerke (alle Siede- und Druckwasser-Reaktoren) werden mit leicht angereichertem Uran betrieben. Nach mehreren hundert bis tausend Betriebstagen werden die Brennstäbe aus-dem Reaktor genommen, weil sie dann entweder durch Anhäufung von Spaltprodukten die Neutronenbilanz zu sehr verschlechtern oder weil sie nicht mehr die notwendige mechanische Stabilität aufweisen. $\mathrm{Zu}$ diesem Zeitpunkt ist häufig das spaltbare Uran-235 noch nicht so weit verbraucht, daß das Uran als Kernbrennstoff wertlos geworden wäre. Das ist erst der Fall, wenn der Anteil des Uran-235 auf 0,4\% oder weniger abgefallen ist 1 ).

Während des Reaktorbetriebes wird neben der Kernspaltung in unvermeidlicher Weise aus dem Uran-238 Plutonium aufgebaut, dessen Isotope Plutonium-239 und Plutonium-241 ebenfalls mit thermischen Neutronen spaltbar sind und dadurch einen wertvollen Kernbrennstoff darstellen:

$$
238 \mathrm{U}+\mathrm{n} \rightarrow 239 \mathrm{U} \stackrel{\beta^{-}, 23 \min }{\longrightarrow} 239 \mathrm{~Np} \stackrel{\beta^{-}, 2,3 \mathrm{~d}}{\longrightarrow} 239 \mathrm{Pu}
$$

(Pu-239: $\alpha$-aktiv, 2,4 $10^{4}$ Jahre Halbwertszeit)

Der Handelspreis des spaltbaren Materials liegt heute bei 52000 DM für $1 \mathrm{~kg} \mathrm{93 \%}$ Uran-235 und DM 170000,- für $1 \mathrm{~kg}$ spaltbares Plutonium *2). Die Kosten der Wiederaufarbeitung betragen rund DM $1 \overline{2} \overline{5}$,-

\footnotetext{
* Mit zunehmendem Anfall zivilen Plutoniums aus den Kernkraftwerken wird dieser Preis voraussichtlich in wenigen Jahren auf ca. DM 40000, - pro kg abfallen. Dieser Preis ist dann der wahre Substitutionswert des Plutoniums für Uran-235 in thermischen Reaktoren.
} 
pro kg Brennstoff. Wirtschaftliche Gründe sprechen deshalb in den meisten Fällen dafür, das spaltbare Material aus den abgebrannten Kernbrennstoffen zurückzugewinnen und den Kernkraftwerken von neuem zuzuführen.

Neben diesem Hauptargument der Wirtschaftlichkeit gibt es noch zwei weitere Gesichtspunkte für die Wiederaufarbeitung.

Die beträchtlichen Mengen radioaktiver Spaltprodukte in den Kernbrennstoffen (20 bis $30 \mathrm{~kg}$ pro Tonne) können zu einer akuten Gefahr für die Umwelt werden, wenn die Kontrolle über sie verloren geht. Durch. die langen Halbwertszeiten einzelner Spaltprodukte ist eine sichere Überwachung über Jahrzehnte hinaus notwendig. Das kann am besten gewährleistet werden, wenn die Spaltprodukte auf ein kleines Volumen konzentriert und an einer zentralen Stelle überwacht werden. Dazu ist ebenfalls eine Wiederaufarbeitung der Kernbrennstoffe notwendig.

Schließlich stellen hochabgebrannte Kernbrennstoffe noch eine Fundgrube interessanter und auch technisch verwertbarer Transuranisotope dar. Die darin vorkommenden Nuclide Neptunium-237 (einige hundert Gramm pro Tonne), Americium-241/143 (ca. $10 \mathrm{~g} / \mathrm{t}$ ) und Curium-244 (ca. $1 \mathrm{~g} / \mathrm{t}$ ) finden entweder direkt oder als Vorprodukt Verwendung beim Bau von Isotopenbatterien.

2. Der nukleare Abbrand und die Plutonium-Erzeugung

Wie erwähnt, läuft neben der Kernspaltung unumgänglich der Neutroneneinfang am Uran-238 ab, der durch die radioaktiven Folgereaktionen bis zum Plutonium-239 führt (Gl. 1).

Bei den konventionellen Uranreaktoren läßt sich in sehr grober Abschätzung damit rechnen, daß etwa $60 \%$ der nutzbaren Neutronen Uran-Spaltungen hervorrufen und etwa $40 \%$ weiterführen zum Plutonium-239. Davon wird etwa die Hälfte während des weiteren Reaktorbetriebes wieder gespalten, der Rest fällt bei der Wiederaufarbeitung an.

Bei den konventionellen Reaktortypen kann heute der Abbrand eines Brennstabes im Durchschnitt auf 2-3 Gewichtsprozent des ursprünglich eingesetzten Gesamturans getrieben werden. Der Gehalt an Plutonium liegt dann bei etwa $0,5 \%$ und kann in günstigen Fällen sogar nahezu $1 \%$ erreichen (Tabelle 1$)^{3)}$.

Bei der langen Verweilzeit im Reaktor von über einem Jahr wird ein Teil des Plutonium-239 unter dem Einfluß der hohen Neutronenflußdichte auch $\mathrm{zu}$ schweren Isotopen bis zur Massenzahl 242 aufgebaut:

$$
\mathrm{Pu}^{239}+\mathrm{n} \rightarrow \mathrm{Pu}^{240}+\mathrm{n} \rightarrow \mathrm{Pu}^{241}+\mathrm{n} \rightarrow \mathrm{Pu}^{242}+\mathrm{n} \rightarrow \mathrm{Pu}^{243} \frac{\mathrm{\beta}^{-}}{5 \mathrm{~h}} \mathrm{Am}^{243}
$$




\begin{tabular}{|c|c|c|c|c|c|c|c|c|c|}
\hline & & VAK & KRB & $\begin{array}{l}\text { DWR } \\
-65\end{array}$ & $\begin{array}{l}\text { DWR } \\
- \text { ORNL }\end{array}$ & $\begin{array}{l}\text { DWR } \\
-70\end{array}$ & $\begin{array}{l}\text { SWR } \\
-70\end{array}$ & $\mathrm{AGR}$ & $\begin{array}{l}\text { HWR } \\
600\end{array}$ \\
\hline \multirow{3}{*}{ 离 } & $\begin{array}{l}\text { Gew. } \% \\
\text { Uran-235 } \\
\end{array}$ & 2,5 & 2,21 & 3,0 & 2,2 & 2,7 & 2,56 & 2,25 & 0,72 \\
\hline & $\begin{array}{l}\text { Gew. \% } \\
\text { Plutonium }\end{array}$ & 0 & 0 & 0 & 0 & 0 & 0 & 0 & 0 \\
\hline & $\begin{array}{l}\text { Abbrand } \\
\text { Gew. \% Uran }\end{array}$ & 1,58 & 1,74 & 2,5 & 2,2 & 2,8 & 2,9 & 2,1 & 0,95 \\
\hline \multirow{2}{*}{ 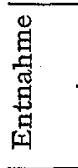 } & $\begin{array}{l}\text { Gew. } \% \\
\text { Uran-235 }\end{array}$ & 1,12 & 0,913 & 1,12 & 0,63 & 0,8 & 0,85 & 0,7 & 0,16 \\
\hline & $\begin{array}{l}\text { Gew. \% } \\
\text { Plutonium }\end{array}$ & 0,56 & 0,62 & 0,93 & 0,62 & 0,44 & 0,8 & 0,55 & 0,39 \\
\hline \multicolumn{10}{|c|}{ Gew. \% gebildetes } \\
\hline Gew. & $\% \mathrm{Pu}-$ Verbraucht & 0,20 & 0,44 & 0,62 & 0,63 & 0,90 & 1,2 & 0,55 & 0,39 \\
\hline
\end{tabular}

$\mathrm{VAK}=$ Versuchs-Atomkraftwerk Kahl, $\mathrm{KRB}=$ Kernkraftwerk Grundremmingen

DWR ( = Druckwasserreaktor-Studien für Großkraftwerke)

- ORNL $=$ von Westinghouse und Oak Ridge Nat. Laboratory bzw. von Siemens-Schuckert-Werke für einen $600(-65)$ und $1000(-70) \mathrm{MW}_{\mathrm{e}}$ Reaktor (Stand 1965)

SWR = Siedewasser-Reaktorstudie für Großkraftwerke der Fa. AFG für eine Reaktorleistung von 600(-65) und 1000(-70) MWe (Stand 1966)

$\mathrm{AGR}=$ Studie eines $1000 \mathrm{MW}$ gasgekühlten Hochtemperatur-Realors der United Kingdom Atomic Energie Autority

HWR $=$ Studie eines $600 \mathrm{MW}_{\mathbf{e}}$-Schwerwasser-Reaktors der Fa. Siemens

or (Die Angaben sind nach der Literaturstelle ${ }^{3)}$ zusammengestellt) 
Plutonium-243 zerfällt mit 5 Std. Halbwertszeit zu Americium-243. Die anderen Plutoniumisotope haben jedoch sehr lange Halbwertszeiten und bleiben erhalten. In der Regel macht der Anteil des spaltbaren Plutonium-239 und Plutonium-241 ungefähr die Hälfte des GesamtPlutoniums aus.

Durch die nicht spaltbaren, , isotopen Verunreinigungen " $\mathrm{Pu}-240 / 242$ ist das ,zivile Plutonium“ nicht mehr ,waffenfähig". Die Spaltbilanz über die Gesamtmenge des Plutoniums ist jedoch immer noch genügend groB, um damit das kostspielige Uran-235 in Kernkraftwerken ersetzen zu können.

In dieser Substitutionsmöglichkeit des Uran-235 liegt die eine Bedeutung des ,zivilen Plutoniums“.

Die andere, noch größere Bedeutung liegt darin, daß es den einzig möglichen Kernbrennstoff für Schnelle Brut-Reaktoren darstellt. In diesem Reaktortyp der Zukunft soll das nicht spaltbare Uran-238 in einem gröBeren Maße in Plutonium umgewandelt werden, als spaltbarer Brennstoff selbst verbraucht wird. Damit wäre die Energieversorgung der Menschheit auf mehr als ein Jahrtausend hinaus sichergestellt, weil sie dann nicht mehr auf Uran-235, sondern auf Uran-238 aufbaut, das in genügender Menge vorhanden ist. Das hierzu notwendige Plutonium muß bis dahin aber aus den Brennelementen der konventionellen thermischen Reaktoren gewonnen werden.

\section{Der Unterschied ziviler und militärischer Wiederaufarbeitung}

Die erste Wiederaufarbeitung von Kernbrennstoffen hatte rein militärischen Grund. Die Tatsache, daß Plutonium-239 einem neuen chemischen Element angehört, eröffnete 1941 für die USA 4) den verhältnismäßig einfachen Weg der chemischen Trennung $\mathrm{zu}$ einem spaltbaren Material. Für militärische Zwecke ist jedoch ein isotopen-reines Plutonium-239 erforderlich. Um den Aufbau höherer Plutoniumisotope zu vermeiden, darf das Uran nur relativ kurze Zeit im Kernreaktor verweilen. Demzufolge ist dann aber auch die Menge der erzeugten Spaltprodukte verhältnismäßig gering.

Bei den zivilen Kraftwerksreaktoren dagegen ist der übergeordnete Gesichtspunkt die Wirtschaftlichkeit der Energieerzeugung. Die Konsequenz daraus ist das Gegenteil, nämlich eine besonders lange Verweilzeit der Brennstäbe im Kernreaktor bei hoher Leistungsdichte.

Für die Wiederaufarbeitung ergeben sich daraus zwei wesentliche neue Merkmale.

a) Die lange Verweilzeit von 500 bis 1000 Tagen im Reaktor bei hoher Leistungsdichte $(15-25 \mathrm{~kW} / \mathrm{kg}$ ) hat einen hohen nuclearen Abbrand zur Folge. Für die Brennstäbe wird dadurch ein chemisch und mechanisch 
besonders stabiles Hüllmaterial erforderlich. Von außen hat die Brennstabhülle länger als ein Jahr dem Kühlmittel bei $200-400^{\circ} \mathrm{C}$ standzuhalten. Von innen ist das Material dem zunehmenden Druck ausgesetzt, der durch die Volumenvermehrung infolge der Uranspaltungen entsteht. Dazu kommt noch die strukturschädigende Wirkung der Reaktorstrahlung. Unter diesen Bedingungen kommen als Hüllmaterial von den nuklear geeigneten Werkstoffen nur chemisch sehr widerstandsfähige $\mathrm{Cr}-\mathrm{Ni}$ Stähle und Zirkon infrage. Um dieses Hüllmaterial aufzuschließen, muß dem Wiederaufarbeitungsproze $\beta$ ziviler Kernbrennstoffe deshalb zuerst ein aufwendiges chemisches oder mechanisches Aufschlußverfahren (Head End) vorausgeschickt werden. Zum Auflösen von Zirkon oder Zirkonlegierungen wird $\mathrm{NH}_{4} \mathrm{~F}+\mathrm{NH}_{4} \mathrm{NO}_{3}$ verwendet (Zirflex-Verfahren). Andere Vorschläge sind ein Lösungsmittelgemisch aus $\mathrm{HF}$ und $\mathrm{H}_{2} \mathrm{O}_{2}$ (Perflex-Verfahren) oder die Verfüchtigung des Zirkons als $\mathrm{ZrCl}_{4}$ mit HCl-Gas bei 300 bis $600^{\circ} \mathrm{C}$ (Zircex-Verfahren). Für die Auflösung von rostfreiem Stahl kann $\mathrm{H}_{2} \mathrm{SO}_{4}$ verwendet werden (Sulfex-Verfahren), Königswasser (Darex-Verfahren) oder ein Gemisch aus $\mathrm{HNO}_{3}+\mathrm{NH}_{4} \mathrm{HF}_{2}$ (Niflex-Verfahren). Als Material für das Auflösegefäß dient beim Zirflex-, Perflex-, Suflex- und Niflex-Verfahren eine gegen Säure besonders widerstandsfähige Nickel-Chrom-Legierung (Nionel). Beim Darex- und ZircexVerfahren ist am besten Titan als Behältermaterial geeignet 5) . . - -

Um den Aufwand der Lagerung oder Verfestigung des gelösten Hüllmaterials zu vermeiden, wurden auch mechanische Verfahren zur Entfernung der Brennstabumhüllung entwickelt ${ }^{6}$ ). In der Praxis hat sich aber nur das Shear- oder Chop-Leach-Verfahren eingeführt. Dabei werden die Brennstäbe durch Abstanzen oder Scheren zerkleinert. Der Kernbrennstoff kann dann mit Salpetersäure ausgelaugt werden. Das Hüllmaterial verbleibt als ungelöster Rückstand. Neben einem großen Durchsatz und dem Anfall des Hüllmaterials in fester Form ist der besondere Vorteil dieses Verfahrens, daß der Auflösevorgang in einem einfachen, rostfreien Stahlbehälter möglich ist.

b) Das andere Merkmal ziviler Wiederaufarbeitung ist durch den mehr als tausendfach höheren Abbrand gegeben. Die Folge davon ist der notwendige Umgang mit entsprechend tausendfach höheren Spaltproduktkonzentrationen und Strahlungsintensitäten, die sich besonders durch strahlenchemische Reaktionen auf die Prozeßchemikalien auswirken.

\section{Die historische Entwicklung}

Die zivile Wiederaufarbeitung benützt heute zu einem großen Teil dieselben chemischen Methoden, die in den nicht-zivilen Anlagen der USA entwickelt worden sind. Der Versuch, durch eine historische Betrachtung 
zu einem Verständnis des heutigen Entwicklungsstandes $\mathrm{zu}$ kommen und darüber hinaus die Tendenzen der Zukunft $z u$ erkennen, muß deshalb auch diesen Entwicklungsbereich in die Betrachtung mit einbeziehen.

\section{Das $\mathrm{BiPO}_{4}$-Fällungsverfahren ${ }^{7,8)}$}

Der erste Wiederaufbereitungsprozeß war ein Fällungsverfahren. Die Entscheidung darüber war getroffen worden nach Vorversuchen über die Plutonium-Abtrennung durch Fällung, durch Lösungsmittelextraktion, durch Verflüchtigung und durch pyrochemische Verfahren. Ausschlaggebend war dabei die unbedingte Notwendigkeit gewesen, unter dem Druck der Kriegsverhältnisse, innerhalb einer festgelegten, kurzen Zeitspanne zumindest einen Teilerfolg zu erzielen. Die Versuchsergebnisse beruhten auf Experimenten, die nur den Bruchteil eines Milligramms an Plutonium zur Verfügung hatten. Unter diesen Voraussetzungen bot damals nur das Fällungsverfahren einigermaßen eine Chance.

Im Fällungsverfahren mußte der erste Niederschlag des Plutonium(III) oder -(IV) in Gegenwart eines großen Überschusses an Uran erfolgen. Um eine Mitfällung des Urans zu vermeiden, war es erforderlich,-in stark-saurer Lösung zu arbeiten. Damit war schon eine großeEinschränkung in den Fällungsreagenzien gemacht. Es kamen nur noch das $\mathrm{LaF}_{3}$ oder einige schwerlösliche Phosphate als Träger der Plutoniumfällung infrage. Unerwarteter Weise zeigte das $\mathrm{BiPO}_{4}$ eine Mitfällung des Plutonium(IV) zu mehr als $98 \%$. Das geringere Korrosionsproblem bei der Phosphatfällung gegenüber der $\mathrm{LaF}_{3}$-Fällung entschied zugunsten des $\mathrm{BiPO}_{4}$. Gegenüber $\mathrm{LaF}_{3}$ zeigt $\mathrm{BiPO}_{4}$ außerdem noch den Vorteil der Löslichkeit in konzentrierter $\mathrm{HNO}_{3}$. Gegenüber anderen schwerlöslichen Phosphaten besitzt $\mathrm{BiPO}_{4}$ außerdem noch eine höhere Dichte und eine bessere Sedimentationsfähigkeit.

Mit einer einmaligen Ausfällung wird von den Spaltprodukten Zirkon und den Seltenen Erden jedoch keine Abtrennung erreicht, weil diese Elemente ebenfalls schwerlösliche Phosphate bilden.

Nach jedem Fällungsschritt und anschließendem Auflösevorgang wurde deshalb das Plutonium(IV) mit Bichromat oder Wismutat zum. löslichen $\mathrm{PuO}_{2}^{2+}$ oxydiert und erneut eine Phosphatfällung durchgeführt. Spalt-Zirkon und -Seltene-Erden gingen dabei wieder in den Niederschlag und das $\mathrm{PuO}_{2}^{2+}$ blieb in Lösung. Beim anschließenden Reduktionsvorgang des Plutoniums wurde Eisen(II) angewendet. Nach zwei solchen Reinigungscyclen folgte ein Konzentrierungsschritt in Form einer $\mathrm{LaF}_{3}$-Fällung. Die hohe Schwerlöslichkeit des $\mathrm{LaF}_{3}$ erlaubt eine entsprechende Verminderung des Lanthanträgers gegenüber dem Wismutträger und läßt eine Anreicherung des Plutoniums auf das 400-fache 
zu. Bei der damit erreichten Konzentration war dann anschließend eine direkte Plutoniumperoxyd-Fällung möglich.

Die erste technische Wiederaufarbeitung nach diesem Prinzip wurde Anfang 1944 in den Clinton-Engineering-Works (später Oak Ridge, Tennessee) verwirklicht und erreichte eine Ausbeute von $50 \%$, die bald auf 80 bis $90 \%$ gesteigert wurde. Die großtechnische Plutoniumproduktion begann aufgrund dieser Erfahrungen um die Jahreswende 1944/45 in den Hanford Anlagen bei Richland, Washington. Das Verfahren wurde dort bis 1951 ausgeüibt.

\section{Die Extraktionsverfahren}

Dem Fällungsverfahren waren einige schwerwiegende Nachteile zu eigen. Durch die mehrfachen Fällungs- und Löseschritte war der Prozeß diskontinuierlich. Bei der notwendigerweise ferngesteuerten Arbeitstechnik aufgrund der radioaktiven Strahlung erschwert aber jede Diskontinuität den Prozeßablauf.

Das Ausgangsmaterial Uran, das den Hauptbestandteil des Prozesses darstellte und alle Prozeß-Chemikalien verließen gemeinsam mit den radioaktiven Spaltprodukten die Anlage als Abfall. Dadurch trat einerseits ein beträchtlicher Materialverlust ein und das radioaktive_Abfallvolumen vergrổ îrte sich erheblich.

In weiterer Sicht war das Fällungsverfahren auch nur ein Teilprozeß, weil er nur auf die Gewinnung des Plutoniums ausgerichtet war. Er war völlig unbrauchbar für die Wiederaufarbeitung von Brennelementen aus anderen Reaktoren. Materialprüfreaktoren werden beispielsweise mit hochangereichertem Uran betrieben und die Wiederaufarbeitung dient hier nur der Rückgewinnung des hochangereicherten Urans und nicht dem Plutonium. Ebenso konnte der Fällungsprozeß nicht angewendet werden zur Gewinnung von spaltbarem Uran-233, das in neutronenbestrahltem Thorium enthalten ist.

Diese Gründe führten deshalb sehr bald zur Entwicklung von kontinuierlich arbeitenden Gesamtprozessen, die neben der Isolierung des Plutoniums auch eine Rückgewinnung des Urans gestatten.

Grundlage dieser Gesamtprozesse wurde die Lösungsmittel-Extraktion. In den USA waren es zuerst Verfahren, die Methylisobutylketon (Hexon) als Extraktionsmittel verwendeten. Später trat an dessen Stelle das Tributylphosphat. In Kanada wurde von Anfang an (1948) ein Extraktionsverfahren mit Tri-glycol-dichlorid benützt. Das englische Wiederaufbereitungsprojekt war anfangs mit dem kanadischen verbunden. In England selbst (ab 1952) kam im ersten Verfahren Dibutylcarbitol zur Anwendung. In der zweiten Phase trat an dessen Stelle das Tributylphosphat. Die Wiederaufarbeitung in Frankreich hat von Anfang 
an (1954) nur Tributylphosphat verwendet. Soweit aus der Entwicklungsgeschichte dieser Verfahren Lehren zu ziehen sind, werden sie im folgenden kurz dargestellt.

\section{a) Die Hexon-Verfahren}

Die anfänglich schwachen Ausbeuten (ca. 50\%) bei den ersten halbtechnischen Versuchen in den Clinton-Works haben zur Entwicklung eines Reserveprozesses auf der Basis der Lösungsmittel-Extraktion geführt. Ziel der Entwicklung war einerseits eine höhere Ausbeute, andererseits eine stärkere Konzentrierung des Plutoniums als beim Fällungs-ProzeB ${ }^{9}$. Als Lösungsmittel wurde Methylisobutylketon $\left(\mathrm{CH}_{3} \mathrm{COC}_{4} \mathrm{H}_{9}\right)$ (Hexon) gewählt. Es ist bemerkenswert, daß diese Wahl nicht nach ausgedehnten Versuchen, sondern nur aufgrund der im Labor schon vorliegenden Erfahrungen getroffen worden ist 10 ).

In den Laboratorien hatte man bis dahin Hexon benützt, das als Lack-Verdünnungsmittel leicht und billig zugänglich war, um das Plutonium aus den Abfallösungen zurückzugewinnen. Neben dem ersten Extraktionsverfahren, das nur auf die Gewinnung des Plutoniums gerichtet war, fand in der Folgezeit das Hexon auch noch in zwei anderen Verfahren eine Anwendung, die nur die Gewinnung von Uran zum Ziele hatten. Bei den Brennelementen aus Materialprüfreaktoren ist nur das angereicherte Uran Gegenstand der Wiederaufarbeitung. Das Plutonium wird dabei wegen seines minimalen Anteils mit den Spaltprodukten verworfen. Der spezielle Extraktionsprozeß für diese Aufgabe führte den Namen Hexon-25 (25 ist eine Deckbezeichnung: Uran-235 hat $Z=92, M=235$ ). Für die Gewinnung von Uran-233 aus neutronenbestrahltem Thorium war ein Hexon-Prozeß unter dem Namen Hexon-23 in Gebrauch (Uran233 hat $Z=92, M=233$ ) 11,12 ).

Die Fähigkeit des Hexons, sowohl das Plutonium als auch das Uran zu extrahieren, führte schließlich auch zu einem Gesamtprozeß für beide Elemente. Plutonium zeigt nur in der Oxidationsstufe +4 und +6 die Extrahierbarkeit mit Hexon, aber nicht in der dreiwertigen Stufe. Dieser Umstand wurde nach einer gemeinsamen Uran-Plutonium-Extraktion für die Trennung beider Elemente ansgenützt. Aus der gemeinsamen organischen Phase wurde das Plutonium durch Reduktion mit Fe(II) selektiv in die wäßrige Phase übergeführt. Aufgrund dieses zusätzlichen Reduktionsschriftes wurde für den Gesamtprozeß die Bezeichnung „Redox-Prozeß ${ }^{\prime \prime}$ geprägt ${ }^{13)}$.

Der Redox-Prozeß löste in den USA den Fällungsproze $B$ ab und wurde 9 Jahre lang (1951-1960) in den Hanford-Anlagen bei Richland, Washington, durchgeführt. In einzelnen Cyclen soll er heute noch bei der Wiederaufarbeitung hochangereicherter Brennstoffe in der Idaho-ChemicalProcessing-Anlage benützt werden. 
Die technische Durchführung des Redox-Prozesses wurde durch folgende drei Eigenschaften des Hexons bestimmt:

1. heftige Nitrierungs- und Oxydationsreaktionen des Hexons bei höherer $\mathrm{HNO}_{3}$-Konzentration.

2. Quantitative Extraktion des Urans und Plutoniums nur in Gegenwart eines Aussalzmittels.

3. Zunehmende Extraktion der Spaltelemente Ruthen, Zirkon und Cer bei steigender Nitrat- oder Salpetersäure-Konzentration.

$z u$ 7. Die heftige Zersetzungs-Reaktion des Hexons mit $\mathrm{HNO}_{3}$ und Spuren $\mathrm{NO}_{2}^{-}$, die ab $2 \mathrm{M} \mathrm{HNO}_{3}$ explosiven Charakter annehmen kann ${ }^{6}$, verbietet die Verwendung von höheren Konzentrationen an Salpetersäure zur Aussalzung. Eine weitere nachteilige Konsequenz daraus ist, daß nach dem Auflöseprozeß der Brennelemente die überschüssige Salpetersäure neutralisiert werden muß. Dadurch kommt eine hinderliche Diskontinuität in den Prozeßablauf. Geht man andererseits von geringeren Säurekonzentrationen aus, so verlangsamt sich entsprechend der Auflöseprozeß.

$z u$ 2. Das Aussalzmittel liegt mit seiner Konzentration nahe an der Sättigung und bleibt in der wäßrigen Phase bei den radioaktiven Spaltprodukten: Dadurch ist es dann nicht mehr möglich, das radioaktive Abfallvolumen durch Aufkonzentrieren zu verkleinern und damit die Lagêrkosten zu verringem. Der Veriust des Aussalzmittels mit den radioaktiven Spaltprodukten bedeutet zusätzlich noch einen unwirtschaftlichen Chemikalienverbrauch.

zu 3. Die mit zunehmendem pH-Wert ansteigende Mitextraktion der Spaltelemente Ruthen, Cer und Zirkon erfordert einen möglichst niedrigen Säuregrad bei der Extraktion. Die Folge ist, daß Plutonium nicht im 4-wertigen, sondern im 6-wertigen Oxidationszustand zur Extraktion vorliegen muß. Andernfalls besteht die Gefahr der Plutonium-Ausscheidung durch Hydrolyse. Vor der Extraktion ist beim Redox-Prozeß deshalb noch ein Oxidationsschritt erforderlich.

Bei der Extraktion wirkt sich ein geringer Säuregrad in einer entsprechend geringeren Extraktionsausbeute aus. In der Praxis wurde ein Kompromiß zwischen Produkt-Ausbeute und Dekontaminationsfaktor gewählt 6).

\section{b) Die TBP-Verfahren}

Tri-n-butylphosphat (TBP, als Anti-Schaum-Mittel und Weichmacher unter den technischen Produkten bekannt), hat seine erste Anwendung als Extraktionsmittel zusammen mit Nitromethan gefunden ${ }^{14)}$. Es war nach einem besonders oxidationsstabilen Extraktionsmittel gesucht worden, das auch der Oxidationskraft von Cer(IV) widerstehen konnte. Bereits von dieser ersten Anwendung her ist auch sein Extraktionsver- 
mögen für Uran und Thorium bekannt. Bei der Wiederaufarbeitung hielt des TBP seinen Einzug aufgrund einer ähnlichen Problemstellung. Der hochradioaktive Abfall des $\mathrm{BiPO}_{4}$-Prozesses sollte auf Uran hin aufgearbeitet werden 15,16,17). Die konzentrierte Ausgangslösung war aber stark salpetersauer, und für diese Bedingung wurde ein Extraktionsmittel für Uran gesucht. Die Wahl fiel dabei auf das TBP, weil dieses Lösungsmittel selbst von konzentrierter $(15 M) \mathrm{HNO}_{3}$ bei Raumtemperatur nur langsam angegriffen wird.

Das zweite wichtige Merkmal des TBP, wenn es als Extraktionsmittel angewendet wird, ist die Notwendigkeit der Verdünnung mit einem inerten Lösungsmittel. In reiner Form ist es eine viscose Flüssigkeit mit einer Dichte von nahezu 1. Für eine befriedigende Phasentrennung soll die TBP-Konzentration in einem inerten Lösungsmittel $40 \%$ nicht überschreiten. Mit einer neutralen wäßrigen Phase bildet sich sonst eine verhältnismäßig stabile Emulsion.

Der Extraktionsvorgang selbst beruht auf der Bildung eines stöchiometrischen TBP-Metall-Komplexes. Die Stöchiometrie zusammen mit der limitierten TBP-Konzentration bedeutet im Vergleich zu Hexon eine erhebliche Einschränkung in der Beladbarkeit der organischen Phase. Bezogen auf gleiche Anlagengröße ergibt sich daraus eine Kapazitätseinbuße um etwa die Hälfte gegenüber Hexon.

Als weitere Nachteile des TBP gegenüber dem Hexon können die Dreiphasenbildung mit vierwertigen Metallen wie $\mathrm{Th}$ und $\mathrm{Pu}$ angeführt werden und die radiolytische Zersetzung zu Produkten, die sich störend auswirken, sowohl auf die Produktausbeute wie auf den Dekontaminationsfaktor.

Die Dreiphasenbildung beruht auf einer beschränkten Löslichkeit der TBP-Metall-Komplexe in aliphatischen Kohlenwasserstoffen. Ein technisches Verfahren wäre bei einer Dreiphasenbildung unmöglich. Sie tritt jedoch erst bei so hohen TBP-Konzentrationen ein, daß sich daraus für die Praxis keine ernsthaften Beschränkungen ergeben.

Das Problem und die Überwindung der störenden radiolytischen TBPZersetzung wird im Abschnitt V, 2, d im Detail behandelt.

Insgesamt betrachtet, hat das TBP aber mehr Vorteile als Nachteile gegenüber dem Hexon. Die Löslichkeit in Wasser (und damit der laufende Lösungsmittelverlust) liegt bei TBP mit $0,4 \mathrm{~g} / 1$ wesentlich niedriger als bei Hexon mit $20 \mathrm{~g} / \mathrm{l}$. Von entscheidender Bedeutung ist jedoch die beträchtliche Stabilität des TBP auch gegenüber starker $\mathrm{HNO}_{3}$. Diese Oxidationsstabilität erlaubt beim Auflöseprozeß starke Salpetersäure zu verwenden und damit auch den Übergang vom Auflöseschritt zum Extraktionsprozeß kontinuierlich zu gestalten. Der zweite noch bedeutendere Gewinn liegt im Fortfall des Aussalzmittels. Die erhöhte Salpetersäurekonzentration reicht aus, um eine vollständige Extraktion des 
Urans und Plutoniums auch ohne Aussalzmittel zu erreichen. Als Folge davon kann das hochradioaktive Abfallvolumen durch Destillation auf etwa 1/25 vermindert werden. Das bedeutet eine erhebliche Einsparung bei der Abfallagerung.

Neben der ursprünglichen Verwendung des TBP zur Rückgewinnung des Urans aus dem $\mathrm{BiPO}_{4}$-Abfall gibt es zum Hexon-25 und Hexon-23Prozeß analoge TBP-Verfahren.

Der TBP-25-Prozeß dient zur selektiven Rückgewinnung von angereichertem Uran (Uran-235: $Z=92, M=235$ ) aus Brennelementen von Materialprüfreaktoren $6,12,17)$.

Der Interim-23-Proze $\beta$ ist für die Gewinnung von Uran-233 $(Z=92$, $M=233$ ) aus Neutronen-bestrahltem Thorium entwickelt worden $6,12,17$ ). Er läßt sich im Gegensatz zum Hexon-23-Verfahren besonders bei sehr kurzzeitig abgeklungenem Thorium verwenden. Interim sollte den provisorischen Charakter des Prozesses andeuten.

Im Thorex-ProzeB wird mit TBP sowohl das Uran-233 als auch das Thorium isoliert $6,12,17,18$ ).

Die Möglichkeit, sowohl Uran als auch Plutonium ohne Aussalzmittel nur durch Anwendung von Salpetersäure zu extrahieren, hat sehr bald zur Entwicklung eines Gesamtprozesses für die Gewinnung von Uran und Plutonium aus abgebrannten Kernbrennstoffen geführt ${ }^{19)}$.

- Zür Unterscheidū̄ng vom Redō̄-PĩozeB uñd dem Uran-Rückgewinnungs-Proze $\beta$ aus $\mathrm{BiPO}_{4}$-Abfall wurde für diesen Gesamtprozeß mit TBP die Bezeichnung „Purex" als Kurzform für Plutonium-UraniumReduktion-Extraktion-Prozeß gewählt.

Der Purex-Proze $B$ ist heute das allgemein eingeführte Verfahren zur Wiederaufarbeitung von Uran-Plutonium-haltigen Kernbrennstoffen ${ }^{6}$, $12,17,20,21,22)$.

\section{c) Andere Extraktionsverfahren}

Neben dem Purex- und Redox-Prozeß sind noch einige andere Extraktionsverfahren zur Wiederaufarbeitung von Kernbrennstoffen entwickelt worden, die aber entweder nach einiger Zeit wieder aufgegeben wurden, oder bis heute noch nicht zu einer technischen Verwirklichung gekommen sind.

\section{Der.TTA-Prozeß 7,17)}

Thenoyl-trifluoraceton (TTA) besitzt für viele Elemente, darunter auch Plütoniüm, ein selektives Extraktionsvermögen. Dementsprechend wurde damit schon 1945 ein Verfahren nur zur Abtrennung des Plutoniums von Uran und den Spaltprodukten entwickelt 7). Durch die hohe Selektivität des TTA würde bei diesem Prozeß ein einziger Extraktionscyclus genügen. Weiterhin sind dazu keine Fremdsalze notwendig, so daß das radio- 
aktive Abfallvolumen wie beim Purex-Prozeß auf ein Minimum konzentriert werden könnte. Die Geschwindigkeit der Komplexbildung und damit auch der Extraktion ist jedoch so langsam, daß die notwendigen Wartezeiten zu einem diskontinuierlichen Prozeßablauf führen. Dieser Nachteil kann auch nicht durch entsprechende Vergrößerungen des Anlagevolumens in Form größerer Zwischentanks ausgeglichen werden, weil damit die Gefahr nuklearer Kritikalität verbunden wäre. Aus diesen Gründen kam der TTA-Prozeß nie zu einer technischen Anwendung.

Der Trigly-Prozeß ${ }^{23}$ )

Während des 2. Weltkrieges scheiterten aus verschiedenen politischen Gründen 24) die Bemühungen der englischen Regierung, am amerikanischen Manhattan-Projekt teilzunehmen. Englische und kanadische Wissenschaftler entwickelten deshalb ein eigenes Aufarbeitungsverfahren von Kernbrennstoffen zur Isolierung von Plutonium. Die Wahl fiel von vornherein auf einen Extraktionsprozeß mit Triglykol-dichlorid (Trigly) $\left(\mathrm{Cl}\left(\mathrm{C}_{2} \mathrm{H}_{4} \mathrm{O}\right)_{2} \mathrm{C}_{2} \mathrm{H}_{4} \mathrm{Cl}\right)$ als Lösungsmittel. Das $\mathrm{Ziel}$ war damals ebenso wie beim $\mathrm{BiPO}_{4}$-Prozeß nur die Isolierung des Plutoniums. Das Uran ging in die radioaktive Abfallösung. Das Trigly hatte zwar den Vorteil der Stabilität gegenüber Salpetersäure. Die Schwierigkeiten waren jedoch dieselben wie beim $\mathrm{BiPO}_{4}$-Prozeß; durch den Anfall großer Mengen nicht konzentrierbarer, radioaktiver Abfallösungen (Uran) war das Verfahren unwirtschaftlich, und die Plutoniumausbeute erreichte nur $90 \%$. Trotzdem wurde das Verfahren in Chalk-River 6 Jahre lang bis 1954 ausgeübt. Dann wurde es durch ein Ionenaustauschverfahren abgelöst. Dabei wurde das Plutonium aus stark-salpetersaurer Lösung wiederholte Male auf Anionenaustauschern adsorbiert. Das reine Ionenaustauschverfahren hatte aber den Nachteil der Diskontinuität und der geringen Kapazität. Schließlich wurde 1956 die Plutoniumgewinnung von der Kanadischen Regierung grundsätzlich eingestellt.

Der Butex-Prozeß 25, 26)

Bei der Planung der Wiederaufarbeitung von Kernbrennstoffen in Großbritannien entschieden sich die englischen Wissenschaftler bei der Wahl zwischen Trigly und Butex (Dibutoxy-diäthyläther) für das Butex. Dieses Lösungsmittel bietet neben einer gemeinsamen Extraktion von Uran und Plutonium auch die erforderliche Stabilität gegenüber Salpetersäure und ermöglicht es, das Aussalzmittel durch Salpetersäure zu ersetzen. Durch Eindampfen läßt sich dadurch das zu lagernde radioaktive Abfallvolumen wieder erheblich verkleinern.

Das Plutonium wurde von Uran aus der gemeinsamen organischen Phase abgetrennt durch eine Reduktion mit Fe(II)-Sulfamat, wie es beim Purex-Prozeß noch eingehend beschrieben wird. 
Ein besonderes Problem stellte die Abtrennung des Spalt-Ruthens von Uran und Plutonium dar. Dafür muBte ein eigener Extraktionscyclus eingeführt werden, bei dem nicht mit Butex, sondern mit TBP extrahiert wurde. Zur Bewältigung des Ruthen-Problems sind im Laufe der Zeit umfangreiche Anstrengungen unternommen worden. Dabei wurde versucht, durch chemische Vorbehandlung der Speiselösung mit Ozon, salpetriger Säure, höherer Temperatur etc. das Ruthen entweder zu verflüchtigen oder in eine nicht extrahierbare Form zu bringen.

Die Versuche blieben jedoch ohne entscheidenden Erfolg.

Diese besondere Schwierigkeit der Dekontamination des Plutoniums und Urans von Ruthen war einer der Hauptgründe, da 3 bei der Planung der neuen Windscale-II Wiederaufarbeitungsanlage das Butex aufgegeben worden ist, zugunsten eines reinen TBP-Prozesses. Der andere Grund für das Verlassen des Butex waren die erheblich höheren Materialkosten des Butex-Verfahrens gegenüber dem vergleichbaren PurexProzeß 27).

\section{Das Eurex-Verfahren 28,29)}

Die Verwendung von Aminen zur Extraktion geht zurück auf die Gewinnung von Uran und Thorium aus Erzen. Umfangreiche Untersuchungen wurden in dieser Richtung durchgeführt $30,31,32,33,34)$.

In der Regel werden dazu tertiäre Amine verwendet, die in Kohlenwasserstoffen gelöst und mit anorganischen Säuren in quarternäre Alkylamoniumsalze tibergeführt worden sind. Solche Lösungen sind in der Lage, entsprechend den analog gebauten Anionenaustauschern Metallsalze in neutraler oder anionischer Form zu binden entsprechend den Gleichungen:

$$
\begin{aligned}
\mathrm{MX}_{\mathrm{n}, \mathrm{aq}}+\mathrm{m} \mathrm{R}_{3} \mathrm{NHX}_{\mathrm{org}} & \rightleftharpoons\left(\mathrm{R}_{3} \mathrm{NH}\right)_{\mathrm{m}} \mathrm{MX} \mathrm{n}_{\mathrm{n}+\mathrm{m}, \mathrm{org}} \\
\mathrm{MX}_{\mathrm{n}+\mathrm{m}}^{\mathrm{m}}+\mathrm{m} \mathrm{H}^{+}+\mathrm{m} \mathrm{R}_{3} \mathrm{NHX} & \rightleftharpoons\left(\mathrm{R}_{3} \mathrm{NH}\right)_{\mathrm{m}} \mathrm{MX} \mathrm{X}_{\mathrm{n}+\mathrm{m}}+\mathrm{m} \mathrm{H}^{+}+\mathrm{mX} \mathrm{X}^{-}
\end{aligned}
$$

Die möglicherweise bei der Wiederaufarbeitung hochabgebrannter Kernbrennstoffe nach dem Purex-Verfahren zu erwartenden Schwierigkeiten durch Radiolyse haben dazu geführt, die Möglichkeiten der Extraktion mit Aminen zu prüfen. Bei einem Gesamtprozeß, bei dem die Rückgewinnung von Uran und Plutonium angestrebt wird, sind die Amin-Verfahren jedoch mit demselben Nachteil belastet wie die Hexon-Verfahren. Um genügend hohe Verteilungskoeffizienten zu erzielen, brauchen die Amine zusätzliche Aussalzmittel, die wiederum das radioaktive Abfallvolumen belasten. Reine Salpetersäure genügt für die Extraktion des Urans nicht. Bei der Wiederaufarbeitung von hoch angereichertem Uran aus Materialprüfreaktoren liegen die Verhältnisse für die Aminextraktion jedoch günstiger. Diese Brennstoffe enthalten zwischen 94 und 97\% Aluminium und 3-6\% reines Uran-235. Damit ist 
nach der Auflösung des Brennelements ohnehin die hohe Aussalzkonzentration gegeben. Für derartige Brennelemente wurde deshalb als Alternative zu dem schon bestehenden Verfahren TBP-25 und Hexon-25 ein Alternativverfahren auf der Basis der Extraktion des Urans mit tertiären Alkylamoniumverbindungen ausgearbeitet (Enriched Uranium Extraction = Eurex) ${ }^{28,29)}$. Der Vorteil der Amin-Extraktion gegenüber schon bestehenden Verfahren wird in günstigeren Dekontaminationsfaktoren und damit in einer geringeren Cyclenzahl des Prozesses gesehen. Innerhalb von zwei Extraktionscyclen sollen Dekontaminationsfaktoren von $10^{6}$ bis $10^{7}$ erreicht werden.

Die Abnahme der Extraktionsfähigkeit von Uran mit steigendem Molekulargewicht der Amine einerseits und die mit abnehmendem Molekulargewicht der Amine zunehmende Löslichkeit in der wäßrigen Phase haben zu Tricaprylamin als Kompromiß geführt. Auch die übrigen Prozeßbedingungen sind weitgehend Kompromisse, denn die Extraktion des Urans und der Spaltprodukte verläuft gleichsinnig unter den verschiedenen Prozeßvariablen wie Aminkonzentration, Säurestärke, Aussalzmittel etc.

Das Problem der Dreiphasenbildung, das bei der Extraktion mit Aminen noch besonders hinzukommt; wurde durch die Wahl eines aromatischen Kohlenwasserstoffs (Trimethylbenzol) umgangen. Bei den TBPProzessen dagegen liegt die Dreiphasenbildung soweit außerhalb der Prozeßbedingungen, daß aromatische Kohlenwasserstoffe, die wegen der möglichen Nitrierungsreaktionen unerwünscht sind, vermieden werden können. Bei der Wahl des Verdünnungsmittels ist die Aminextraktion ebenfalls nicht unabhängig, weil durch sie im Gegensatz zum TBP, die Extraktionseigenschaften beeinflußt werden ${ }^{29}$ ).

Zweifellos ein Vorteil gegenüber TBP sind die den Prozeß nicht störenden Radiolyse-Produkte (sekundäre und primäre Amine). Dabei bleibt aber noch die Frage offen, wie weit das Extraktionsmittel unter den realen ProzeBbedingungen einer hohen Dosisleistung auf die Dauer zu widerstehen vermag.

\section{Die Chemie des Purex-Verfahrens}

1. Der Extraktionsvorgang bei Uran, Plutonium, $\mathrm{HNO}_{3}$ und einigen Spaltprodukten

Der Extraktionsvorgang mit TBP beruht auf der Bildung einer stöchiometrischen Komplexverbindung, bei der zwei oder drei TBP-Moleküle über die polare Phosphorylgruppe an das zentrale Metallatom gebunden sind. Die Butylgruppen bringen die organische Löslichkeit des Komplexes, der als neutrales Nitrat in die organische Phase übertritt. $\mathrm{Zu}$ 
einer Komplexbildung dieser Art sind besonders die vierwertigen Metallnitrate der Aktinoide befähigt.

Durch die Bildung einer stöchiometrischen Verbindung läßt sich der Extraktionsvorgang mit Reaktionsgleichungen beschreiben, und das Massenwirkungsgesetz kann Auskunft geben über den Einfluß der beteiligten Komponenten auf den Extraktionsvorgang.

$$
\begin{aligned}
& \mathrm{UO}_{2, \mathrm{aq}}^{2+}+2 \mathrm{TBP}_{\text {org }}+2 \mathrm{NO}_{3, \mathrm{aq}}^{-} \rightleftharpoons \mathrm{UO}_{2}\left(\mathrm{NO}_{3}\right)_{2} \mathrm{TBP}_{2, \text { org }} \\
& \mathrm{Pu}_{\mathrm{aq}}^{4+}+2 \mathrm{TBP}_{\text {org }}+4 \mathrm{NO}_{3, \mathrm{aq}}^{-} \rightleftharpoons \mathrm{Pu}\left(\mathrm{NO}_{3}\right)_{4} \mathrm{TBP}_{2, \text { org }} \\
& \mathrm{Zr}_{\mathrm{aq}}^{4+}+2 \mathrm{TBP}_{\text {org }}+4 \mathrm{NO}_{3, \mathrm{aq}}^{-} \rightleftharpoons \mathrm{Zr}\left(\mathrm{NO}_{3}\right)_{4} \mathrm{TBP}^{2}{ }_{\text {,org }}
\end{aligned}
$$

Das TBP ist über die Phosphorylgruppe auch zur Ausbildung einer Wasserstoffbrückenbindung fähig ${ }^{35}$. Bei starken Säuren wie $\mathrm{HClO}_{4}$, $\mathrm{HJ}, \mathrm{HCl}$ erfolgt die Extraktion des Anions über die Bindung eines hydratisierten Hydroxonium-Ions an das TBP in der Form: TBP $\cdot \mathrm{H}\left(\mathrm{OH}_{2}\right)_{4}^{+} \mathrm{X}-$. Die Salpetersäure tritt in diesem Rahmen nicht als starke Säure auf, sondern wird als unhydratisiertes $\mathrm{TBP} \cdot \mathrm{HNO}_{3}$ extrahiert. Zur Verschiebung des Extraktionsgleichgewichtes hin zur organischen Phase sind deshalb nicht extrahierbare Nitrate wie $\mathrm{Al}\left(\mathrm{NO}_{3}\right)_{3}$ etc. besser geeignet als $\mathrm{HNO}_{3}$. Solche Salze sind jedoch nur bei sehr schwach extrahierbaren Elementen wie Thorium oder Americium erforderlich. Zur Extraktion von Uran(VI) und Plutonium(IV) oder Pu(VI) genügt die Aussalzwirkund der Salpetersäure, Abb. 1.

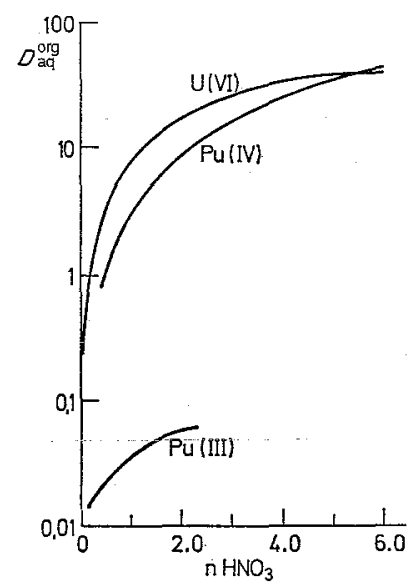

Abb. 1. Der Verteilungskoeffizient $D_{\mathrm{aq}}^{\text {org }}$ von $\mathrm{U}(\mathrm{VI}), \mathrm{Pu}(\mathrm{IV})$ und $\mathrm{Pu}(\mathrm{III})$, einzeln in Lösung in Abhängigkeit von der Salpetersäurekonzentration im System Dodekan$30 \mathrm{vol} \%$ TBP/HNO 3 . Nach J. F. Flagg ${ }^{12)}$ 
Von den Spaltprodukten werden auch noch Zirkon, Niob und Ruthen von TBP gebunden und extrahiert. Bei Zirkon und Niob erfolgt in stark saurem Medium die Bindung des TBP in gleicher Weise wie bei Uran und Plutonium in der ersten Ligandensphäre ${ }^{36)}$ (Gl. 5).

Beim Ruthen ist der Extraktionsvorgang noch nicht so eindeutig geklärt. Der größte Teil des Ruthens liegt in salpetersaurem Medium in Form von verschiedenen Nitrato- (und Nitro)-Komplexen des Nitrosylruthen(III)-Kations vor 37,38,39,40). Bei Änderung der Salpetersäurekonzentration verschieben sich die Gleichgewichte der verschiedenen Ligandenkonfigurationen im Zeitraum von wenigen Minuten bis einigen Tagen:

$$
\begin{gathered}
\mathrm{RuNO}\left(\mathrm{H}_{2} \mathrm{O}\right)_{4}^{3^{+}} \stackrel{\mathrm{HNO}_{3}}{\rightleftharpoons}\left(\mathrm{RuNO}\left(\mathrm{NO}_{3}\right)\left(\mathrm{H}_{2} \mathrm{O}\right)_{3}\right)^{2+} \stackrel{\mathrm{HNO}_{3}}{\rightleftharpoons}\left(\mathrm{RuNO}\left(\mathrm{NO}_{3}\right)_{2}\left(\mathrm{H}_{2} \mathrm{O}\right)_{2}\right)^{+} \stackrel{\mathrm{HNO}_{3}}{\rightleftharpoons} \\
\left(\mathrm{RuNO}_{3}\left(\mathrm{NO}_{3}\right)_{3}\left(\mathrm{H}_{2} \mathrm{O}\right)\right) \stackrel{\mathrm{HNO}_{3}}{\rightleftharpoons}\left(\mathrm{RuNO}\left(\mathrm{NO}_{3}\right)_{4}\right)^{-}
\end{gathered}
$$

Dabei ist die trans-Stellung zur Nitrosylgruppe besonders labil und deshalb in der gegebenen Formulierung nicht erfaBt. Die Extraktion des Ruthen $36,41,42$ ) erfolgt zuerst vermutlich über labile Bindungsformen des TBP entweder in der trans-Stellung oder über Wasserstoffbrückenbindung in der 2. Ligandensphäre. Darauf weisen der negative Temperaturkoeffizient der Extraktion und der Verdrängungseffekt durch Salpetersäure hin. Durch einen Ligandenaustausch kann das TBP dann möglicherweise auch in die stabile, erste Ligandensphäre übertreten. Es wird angenommen ${ }^{41}$, daß die Tetra- und Tri-Nitrato-Komplexe die besonders extraktionsfähigen Formen darstellen.

Für die Dekontamination des Urans und Plutoniums, die mit der Extraktion erreicht werden soll, bedeutet das zeitlich veränderliche Extraktions-Verhalten des Ruthens eine besondere Erschwernis.

\section{Die Mehrkomponentenextraktion}

Unter den realen Prozeßverhältnissen wird nicht eine einzelne Komponente extrahiert, sondern es liegt ein Mehrkomponentensystem vor, in dem Uran, Plutonium und die Spaltprodukte Zirkon, Niob und Ruthen um den Komplexliganden TBP konkurrieren. Das Extraktionsverhalten dieser Stoffe in Abhängigkeit von gleichzeitig anwesendem Uran ist in Abb. 2 dargestellt. Die Abnahme der Verteilungskoeffizienten mit zunehmender Uransättigung zeigt, wie Uran gegenüber den anderen Extraktionspartnern eine deutlich verdrängende Wirkung ausübt.

Die Ursache für die Abnahme des Verteilungskoeffizienten wurde im Fall des Plutonium(IV) untersucht ${ }^{43}$ ). Sie beruht auf einer größeren 


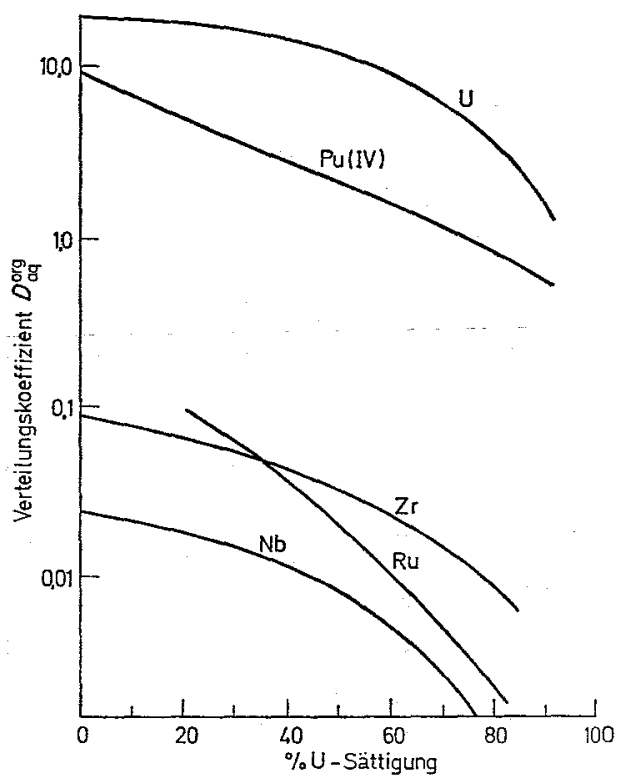

Abb. 2. Einfluß der TBP-Absättigung durch Uran auf die Verteilungskoeffizienten von trägerfreiem PuIV, $\mathrm{Zr}, \mathrm{Nb}, \mathrm{Ru}$ bei $30 \% \mathrm{TBP}=1,1$ Molar $(100 \%$ Uran-Sättigung $=0,55 \mathrm{M}$ Uran)

Phasenübergangsgeschwindigkeit des Urans bei der Hin- und einer geringeren bei der Rückextraktion, Abb. 3. Das Uran läuft dem Plutonium bei der Hin-Extraktion mit einer etwa zweifach größeren Geschwindigkeit in die organische Phase voraus. Umgekehrt ist bei der RückExtraktion die Übergangsgeschwindigkeit des Plutoniums ein mehrfaches größer als bei Uran.

Die Absättigung des TBP durch Uran ist also ein wesentlicher Faktor in der Extraktionsführung. Sie beeinflußt sehr stark die Uran- und Plutoniumausbeute und steuert auch den Dekontaminationsfaktor.

\section{Die extraktive Uran-Plutonium-Trennung}

Plutonium(III) besitzt in TBP einen sehr geringen Verteilungskoeffizienten (Abb. 1). Dadurch bietet sich die Möglichkeit einer reduktiven Uran-Plutonium-Trennung während der Extraktion. Auch eine Trennung durch selektive Komplexbildner wäre möglich 44,45). In der Technik wird bisher jedoch nur die Reduktionsmethode verwendet.

Unter den für die Plutonium(IV)-Reduktion möglichen Reagenzien (Hydrazin, Hydroxylamin, Sulfit, Ascorbinsäure, Hydrochinon, Eisen(II) 


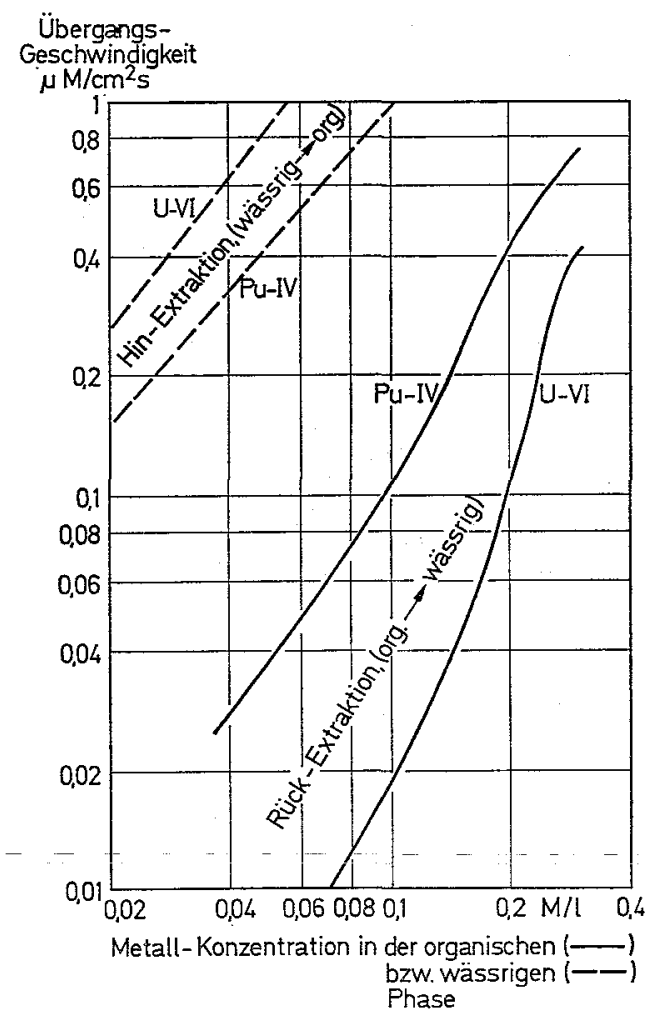

Abb. 3. Die Übergangsgeschwindigkeiten von Uran und Plutonium bei der Hin- und Rück-Extraktion im System 20\% TBP/3n $\mathrm{HNO}_{3}$ als Funktion der Metall-Konzentration in der organischen (-) bzw. wäßrigen (-- ) Phase. Nach L. Finsterwalder 43).

und Uran(IV) zeigen nur das Eisen(II) und Uran(IV) eine genügend schnelle Reaktion unter den Bedingungen, die der Extraktionsprozeß erfordert.

Ein Problem stellt die Stabilisierung des Reduktionsmittels und auch des Plutonium(III) gegenüber der salpetersauren Lösung dar. Der geschwindigkeitsbestimmende Schritt bei der Oxidation ist die Elektronenaufnahme durch $\mathrm{NO}_{2}$ :

$$
\mathrm{Fe}^{2+}+\mathrm{NO}_{2} \rightleftharpoons \mathrm{Fe}^{3+}+\mathrm{NO}_{\overline{2}}
$$

Durch rasche Reaktion des Nitrits mit Salpetersäure induziert jedes gebildete $\mathrm{NO}_{2}^{-}$die Bildung von 2 weiteren Molekülen $\mathrm{NO}_{2}$ :

$$
\mathrm{NO}_{2}^{-}+\mathrm{NO}_{3}^{-}+2 \mathrm{H}^{+} \rightleftharpoons 2 \mathrm{NO}_{2}+\mathrm{H}_{2} \mathrm{O}
$$


Das Anschwellen dieser autokatalytischen Reaktion läßt sich verhindern durch Unterbrechung der Reaktionskette am Nitrit. Von den untersuchten Abfängern für salpetrige Säure (Harnstoff, Formamid, Acetamid, Methylamin, Phenylhydrazon, Sulfamat und Hydrazin) erwiesen sich jedoch nur Sulfamat und Hydrazin als schnell genug wirksam, um die Oxidation des Eisen(II) oder Uran(IV) zu verhindern. Das Reduktionsreagenz ist deshalb entweder $\mathrm{Fe}\left(\mathrm{SO}_{3} \mathrm{NH}_{2}\right)_{2}$ oder $\mathrm{U}\left(\mathrm{NO}_{3}\right)_{4}+$ $\mathrm{H}_{2} \mathrm{NNH}_{2} \cdot \mathrm{HNO}_{3}$. $\mathrm{Fe}\left(\mathrm{SO}_{3} \mathrm{NH}_{2}\right)_{2}$ enthält neben dem reduzierenden Kation Eisen(II) im Anion zugleich den Stabilisator gegenüber der salpetersauren Lösung. Mit Hydrazin beim Uran(IV)-Nitrat wird auch noch die Einführung des Prozeß-fremden Sulfat-Ions vermieden.

Beide Stabilisatoren sind jedoch im organischen Medium nicht löslich. Die Folge ist, daß die in der organischen Phase noch vorhandenen, geringen Mengen an Plutonium(III) ungeschützt der Oxidation durch $\mathrm{NO}_{2}$ ausgesetzt sind. Dadurch kommt es zu einem Cyclus aus teilweiser Reoxidation des Plutonium(III) in der organischen Phase mit anschließender erneuter Reduktion. Die Folge ist ein überstöchiometrischer Bedarf an Reduktionsmittel, der zwischen dem 2- und 15fachen des Plutoniumäquivalents liegt.

In der Technik war ursprünglich nur $\mathrm{Fe}\left(\mathrm{SO}_{3} \mathrm{NH}_{2}\right)_{2}$ im Gebrauch. Mit der Entwicklung geeigneterer Methoden zur Herstellung größerer Mengen Uran(IV) 46,47) hat sich dieses Reduktionsmittel aber auch in den industriellen Anlagen eingeführt (bei Eurochemic in Belgien, Marcoule und Cap La Hague in Frankreich). Gegenüber dem Eisen(II) ist Uran(IV) in der organischen Phase löslich. Das stärkere Redoxpotential des Uran(IV) im Vergleich zu Eisen(II) kommt dabei aber nicht zur Auswirkung. Im Gegenteil, die effektive Reduktionsgeschwindigkeit ist dadurch geringer 45,48 ). Andererseits führt aber auch die gegen Oxidation ungeschützte Exposition des Uran(IV) in der organischen Phase nicht zu einem erhöhten Uran(IV)-Verbrauch ${ }^{49,50,51)}$.

\section{Das technische Verfahren}

25 Jahre nach der ersten Wiederaufarbeitung von Kernbrennstoffen wenden heute alle in Betrieb befindlichen Anlagen die Lösungsmittelextraktion an und benützen dabei ohne Ausnahme eine der Varianten des Purex-Prozesses mit Tributylphosphat. Auch bei allen heute geplanten Anlagen soll, mit einer Ausnahme (Eurex), in derselben Weise verfahren werden (Tabelle 2).

Die ausnahmslose Benützung der Lösungsmittelextraktion, zumindest im ersten hochradioaktiven Cyclus ergibt sich aus den folgenden Erfordernissen, die bei einer Wiederaufarbeitung hochradioaktiver Brennstäbe erfüllt werden müssen: 
ขั Tabelle 2. Die in Betrieb oder Bau befindl. technischen (und halbtechnischen). Wiederaufarbeitungsanlagen. TBP $=$ Tributylphosphat, AA $=$ Anionenaustauscher, SiG $=$ Silikagel, Hex $=$ Hexone, $T L A=$ Trilaurylamin, $T C A=$ Tricaprylamin. $\square$ umfaßt einen Extraktionscyclus, der mit Uron-und/oder Plutonium in wäßriger Lösung beginnt und wieder in wäßriger Lösung endet

\begin{tabular}{|c|c|c|c|c|c|}
\hline Land & $\begin{array}{l}\text { Bezeichriung } \\
\text { Ort }\end{array}$ & $\begin{array}{l}\text { Cyclusfolge } \\
\text { Prozeßmedium }\end{array}$ & $\begin{array}{l}\text { Eigentümer } \\
\text { Betreiber }\end{array}$ & Kapazität & Inbetriebnahme \\
\hline \multirow{6}{*}{ USA } & $\begin{array}{l}\text { SRP } \\
\text { Savanna'h } \\
\text { River }\end{array}$ & $\begin{array}{l}I B P P-P A \\
I B P-P B\end{array}$ & $\begin{array}{l}\text { USAEC } \\
\text { Union Carbide }\end{array}$ & $9 \mathrm{t} / \mathrm{d} ?$ & 1954 \\
\hline & $\begin{array}{l}\text { Purex } 200 \mathrm{E} \\
\text { Hanford }\end{array}$ & 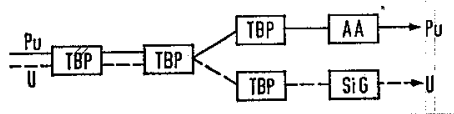 & $\begin{array}{l}\text { USAFC } \\
\text { Batelle }\end{array}$ & $10 \mathrm{t} / \mathrm{d} ?$ & 1956 \\
\hline & $\begin{array}{l}\text { ICCP } \\
\text { Idaho Falls }\end{array}$ & - Hex. -- Hex $\rightarrow$ U-235 & $\begin{array}{l}\text { USAEC } \\
\text { Phillips }\end{array}$ & $1,2 \mathrm{t} / \mathrm{d}$ & 1953 \\
\hline & $\begin{array}{l}\text { West Valley Plant } \\
\text { Staat New York }\end{array}$ & 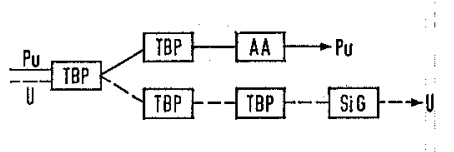 & $\begin{array}{l}\text { Aktiengesell. } \\
- \text { Nuclear Fuel } \\
\text { Service }\end{array}$ & $1 \mathrm{t} / \mathrm{d}$ & 1966 \\
\hline & $\begin{array}{l}\text { Midwest Fuel } \\
\text { Recovery Plant } \\
\text { Illinois }\end{array}$ & 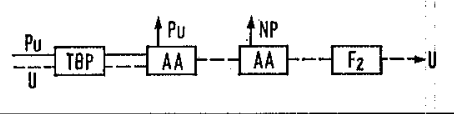 & $\begin{array}{l}\text { General Electric } \\
\text { General Electric }\end{array}$ & $1 \mathrm{t} / \mathrm{d}$ & $1970 / 71$ \\
\hline & $\begin{array}{l}\text { Barnwell Nuclear } \\
\text { Fuel Plant } \\
\text { South Carolina }\end{array}$ & 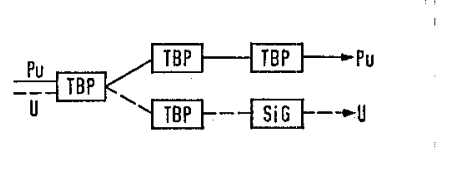 & $\begin{array}{l}\text { Allied Chemical } \\
\text { Corporation } \\
\text { - Allied Chemical } \\
\text { Corporation }\end{array}$ & $5 \mathrm{t} / \mathrm{d}$ & ca. 1975 \\
\hline
\end{tabular}


ARCO Atlantic

ARCO

Atlantic

$5 \mathrm{t} / \mathrm{d}$

1974

South Carolina

Richfield Comp.

\begin{tabular}{|c|c|c|c|c|c|}
\hline England & Windscale II & 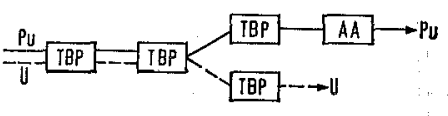 & $\begin{array}{l}\text { UKAEA } \\
\text { UKAEA }\end{array}$ & $\geqslant 5 \mathrm{t} / \mathrm{d}$ & 1964 \\
\hline \multirow{2}{*}{ Frankreich } & $\begin{array}{l}\text { UP } 1 \\
\text { Marcoule }\end{array}$ & $\frac{P u}{U}=T$ TBP & $\begin{array}{l}\mathrm{CEA} \\
\mathrm{CEA}\end{array}$ & $1-2 t / d$ & 1958 \\
\hline & $\begin{array}{l}\text { UP } 2 \\
\text { La Hague }\end{array}$ & $\underset{T B P}{ } \underset{U}{I L A} \rightarrow P_{u}$ & $\begin{array}{l}\text { CEA } \\
\text { CEA }\end{array}$ & $3-4 t / d$ & 1966 \\
\hline Belgien & Eurochemic Mol & 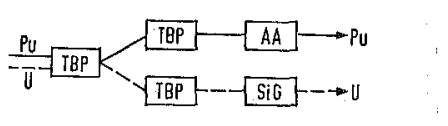 & $\begin{array}{l}\text { OECD } \\
\text { Eurochemic }\end{array}$ & $0,4 \mathrm{t} / \mathrm{d}$ & 1966 \\
\hline Italien & $\begin{array}{l}\text { EUREX } \\
\text { Saluggia }\end{array}$ & $U-235-I C A--I C A-U-235$ & $\begin{array}{l}\text { CNEN } \\
\text { CNEN }\end{array}$ & $\begin{array}{l}\text { 3-6 MTR- } \\
\text { Elemente/d }\end{array}$ & \\
\hline
\end{tabular}


Tabelle 2 (Fortsetzung)

\begin{tabular}{|c|c|c|c|c|c|c|}
\hline Land & $\begin{array}{l}\text { Bezeichnung } \\
\text { Ort }\end{array}$ & $\begin{array}{l}\text { Cyclusfolge } \\
\text { Prozeßmedium }\end{array}$ & i & $\begin{array}{l}\text { Eigentümer } \\
\text { Betreiber }\end{array}$ & Kapazität & Inbetriebnahme \\
\hline Indien & Trombay & 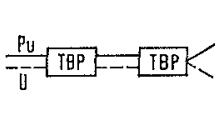 & & $\begin{array}{l}\text { IAEC } \\
\frac{-}{\text { IAEC }}\end{array}$ & $0,25 \mathrm{t} / \mathrm{d}$ & 1965 \\
\hline Japan & Tokai Mura & TBP & 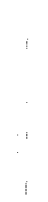 & $\begin{array}{l}\text { Jap. Atomic } \\
\text { Fuel Co. } \\
\text { Jap. Atomic } \\
\text { Fuel Co. }\end{array}$ & $0,7-1 \mathrm{t} / \mathrm{d}$ & ca. 1971 \\
\hline BRD & $\begin{array}{l}\text { WAK } \\
\text { Karlsruhe }\end{array}$ & $\frac{P_{U}}{=T}=\frac{T B P}{T P P}$ & i & $\begin{array}{l}\mathrm{BMwF} \\
\overline{\mathrm{GWK}}\end{array}$ & $175 \mathrm{~kg} / \mathrm{d}$ & 1970 \\
\hline
\end{tabular}


1. Die hohe Radioaktivität erfordert aus Strahlenschutzgründen eine ferngesteuerte und möglichst auch kontinuierliche Betriebsweise.

2. Der hohe Materialwert des rückzugewinnenden Kernbrennstoffes verlangt quantitative Ausbeuten.

3. Die hohe Reinheitsanforderung an die Produkte gegenüber mehr als 30 verschiedenen Elementen in Form radioaktiver Spaltprodukte erfordert eine sehr hohe Selektivität der Trennung.

Von allen Trennverfahren kann noch am besten die Lösungsmittelextraktion alle diese Erfordernisse gleichzeitig erfüllen. Sie läßt sich kontinuierlich und fernbedient steuern. Bei multiplikativer Arbeitsweise erfüllt sie gleichzeitig auch noch die Bedingungen der hohen Ausbeute und der hohen Selektivität. Die hohen Betriebsmittelkosten, die einer breiteren Anwendung der Lösungsmittelextraktion sonst in der Technik im Wege stehen, fallen neben dem hohen Materialwert des Produktes (Plutonium und angereichertes Uran) nicht ins Gewicht.

1. Die Anordnung der Cyclen

Der Kern des Purex-Prozesses besteht aus 2 Extraktionscyclen für jedes Element, Abb. 4. Der gemeinsame Cyclus dient der Uran-PlutoniumTrennung und gleichzeitig einer groben Abtrennung der Spaltprodukte. Die beiden folgenden Cyclen gehören zur weiteren Reinigung der getrennten Produkte.

Zwei Extraktionscyclen mit TBP reichen jedoch in der Regel nicht aus, um die hohen Reinheitsforderungen $\left(D F \sim 10^{7}\right)^{\text {a) }}$ an das Uran und Plutonium, besonders gegenüber den Spaltprodukten Zirkon, Niob und Ruthen zu erfüllen. Weiterhin sind bei nur 2 Cyclen auch der Prozeßdurchführung sehr enge Schranken gesetzt 27). Beispielsweise ist dann,

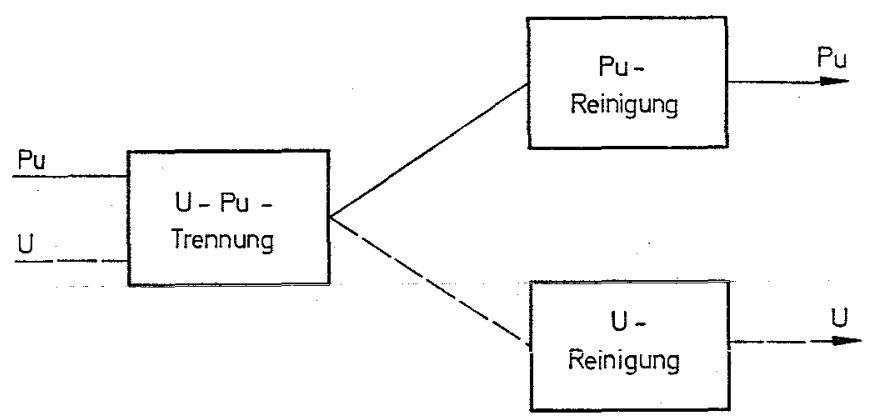

Abb. 4. Prinzip des Purex-Prozesses

a) Dekontaminationsfaktor $\mathrm{DF}=\frac{\text { Radioaktivität/Produkteinheit vor der Dekontamination }}{\text { Radioaktivität/Produkteinheit nach }}$ 
um den erforderlichen Dekontaminationsgrad zu erreichen, eine hohe TBP-Sättigung mit Uran erforderlich. Das setzt wiederum auch eine hohe Konzentration in der wäßrigen Phase voraus und erfordert $Z$ wischencyclus-Verdampfer. Zusätzlich müßten auch umfangreichere Recyclierungs-Einrichtungen für den Fall von Fehlchargen vorgesehen werden. Einsparungen in der direkten Prozeßlinie würden also zusätzliche Ausgaben in den Nebeneinrichtungen erfordern. Außerdem bleibt auch wenig Spielraum für die Verarbeitung zukünftiger Brennstoffe mit noch höherem Abbrand. Aus diesem Grunde findet man in den technischen Anlagen (Tabelle 2) meistens noch einen dritten Reinigungscyclus vor- oder nachgeschaltet und zur Feinreinigung der Produkte noch zusätzlich einen Anionenaustauscher für Plutonium und eine Silicagel-Säule für Uran. $A n l a B$ für den zusätzlichen Aufwand einer Ionenaustauscher- und $A b-$ sorber-Säule ist die schon erwähnte schlechte Dekontamination von Zirkon, Niob und Ruthen.

Geht man davon aus, daß für den erforderlichen Dekontaminationsfaktor von $10^{7}$ außer der Feinreinigung noch 3 Extraktionscyclen erforderlich sind, so bietet ein dem Trennungscyclus vorausgeschickter ,Kodekontaminationscyclus" den Vorteil eines geringeren Apparateaufwandes. Der dritte Cyclus nachgeschaltet, würde den Aufwand im Uran- und Plutonium-Strom jeweils getrennt erfordern, allerdings in kleinerer Dimension.

Hält man sich aber die Ursache des dritten Dekontaminationscyclus vor Augen, nämlich nur die spezielle Dekontamination von Zirkon, Niob und Ruthen, so ergibt sich noch eine Alternative mit nur zwei Cyclen, aber erhöhter Extraktionstemperatur (Tabelle 2, Eurochemic, WAK).

\section{Das Verfahren mit erhöhter Temperatur}

Wie Abb. 5 zeigt, erhöht sich der Dekontaminationsfaktor gegenüber Ruthen fast um den Faktor 100, wenn die Extraktionstemperatur auf $70^{\circ} \mathrm{C}$ gesteigert wird 52). Bei über $60^{\circ} \mathrm{C}$ fällt jedoch der Dekontaminationsfaktor gegenüber Zirkon und Niob in ähnlich starkem Maße ab, so daß der am Ruthen gewonnene Reinheitsfaktor dadurch wieder aufgehoben wird. Die günstigste Temperatur zur Erhöhung des Dekontaminationsfaktors gegenüber beiden Elementen liegt zwischen 50 und $60^{\circ} \mathrm{C}$. Die Ursache für die schwindende Extraktionsfähigkeit des Spalt-Ruthens mit ansteigender Temperatur dürfte in der chemischen Bindungsform des TBP an das Ruthen zu suchen sein. Sehr wahrscheinlich handelt es sich, vgl. III, 1, um Wasserstoff-Brückenbindungen zwischen dem TBP und den Aquo- oder Hydroxo-Liganden der Ruthen-nitrato-Komplexe. Mit steigender Temperatur spalten sich diese Bindungen auf, und das Ruthen verliert dadurch seine organische Löslichkeit. Für diese Inter- 
pretation spricht auch die Beobachtung, daß die Zirkon-Niob-Aktivität dann ebenfalls mit zunehmender Temperatur denselben Extraktionsverlust erleidet wie Ruthen, wenn bei hoher Nitratkonzentration die Säurestärke von 3 auf $0,05 \mathrm{n}$ gesenkt wird (Abb. 6) ${ }^{52)}$. Wegen der gleichbleibenden Nitratkonzentration ist dieser Effekt nur mit der Hydrolyse des Zirkon und Niob in Zusammenhang zu bringen. Vermutlich wird in diesem Fall das TBP in ähnlicher Weise wie an das Ruthen über Wasserstoff-Brückenbindungen an die teilweise hydrolysierten Kationen gebunden. Durch erhöhte Temperatur wird diese Bindung wieder leicht getrennt und die organische Löslichkeit geht verloren.

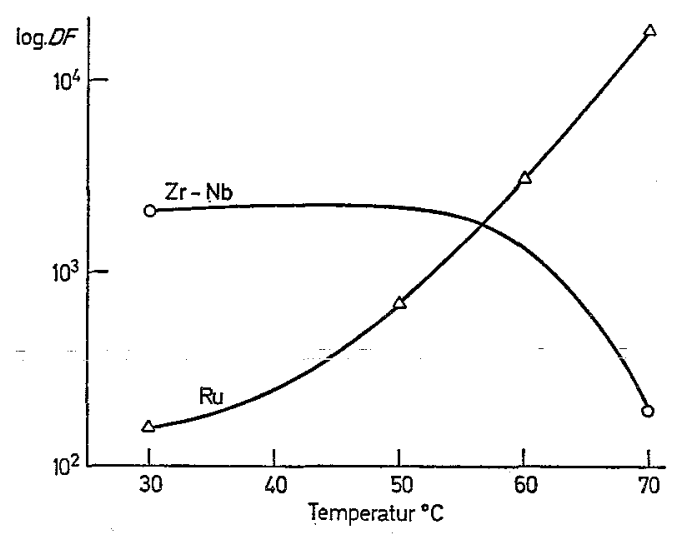

Abb. 5. Dekontaminationsfaktoren gegenüber Zirkon, Niob und Ruthen in Abhängigkeit von der Extraktionstemperatur unter den Bedingungen des 1. Cyclus im Purex-Prozeß (3n $\left.\left.\mathrm{HNO}_{3}\right){ }^{52}\right)$

Auch bei der Abtrennung des Plutoniums vom Uran ist eine erhöhte Betriebstemperatur von Vorteil. Die Rückextraktion, sowohl des Plutoniums als auch der verunreinigenden Spaltprodukte aus dem organischen Uranstrom, wird durch erhöhte Temperatur begünstigt. Für das Plutonium ergibt sich aus der verbesserten Rückextraktion die Möglichkeit, in der wäBrigen Phase die Konzentration zu erhöhen. Die gleichzeitige Verbesserung des Dekontaminationsfaktors für Uran geht zwar auf Kosten der Reinheit des Plutoniums. Dieser Umstand ist jedoch nicht sehr störend, weil durch den nachgeschalteten Anionenaustauscher noch eine genügend hohe Dekontaminationskapazität für Plutonium vorhanden ist (DF ca. 500). Der erhöhte Spaltproduktübergang, zusammen mit dem Plutonium in die wäßrige Phase, ist sogar ein willkommener Faktor 
hinsichtlich der Betriebssicherheit. Bei der ,späten“'Uran-PlutoniumTrennung ist ein wesentliches Argument für den vorgeschalteten Kodekontaminationscyclus das verminderte Aktivitätsrisiko im Uran-Strom.

Eine ähnliche Sicherheit wird auch durch die Plutonium-Abtrennung bei erhöhter Temperatur erreicht. Falls ein Aktivitätsdurchbruch im ersten Extraktor stattfindet, wird die Hauptmenge der Spaltprodukte im folgenden Extraktor, der an sich der Uran-Plutonium-Trennung dient, zusammen mit dem Plutonium wieder aus dem anteilmäßig sehr viel größeren und deshalb umständlicher zu recyclierenden Uran-Strom entfernt 53).

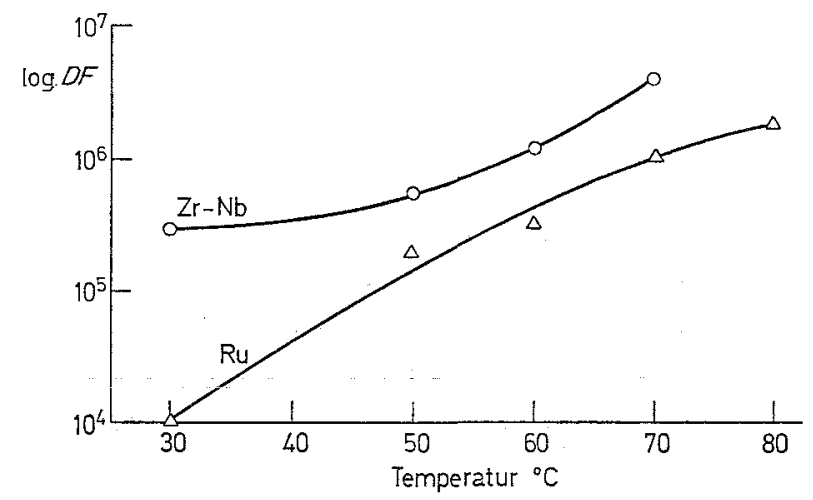

Abb. 6. Dekontaminationsfaktoren gegenüber Zirkon, Niob und Ruthen bei $0,05 \mathrm{n}$ $\mathrm{HNO}_{3}$ und $1,2 \mathrm{n} \mathrm{Al}\left(\mathrm{NO}_{3}\right)_{3}$ in Abhängigkeit von der Temperatur 52)

Die Variante des Purex-Verfahrens mit erhöhter Betriebstemperatur gestaltet sich demnach folgendermaßen: (Abb. 7) Der erste Extraktionsschritt in der Extraktionsbatterie HA (Hot Extraktor A) erfolgt bei Normaltemperatur. Der Extraktion des Ruthens wird dabei durch erhöhte Salpetersäurekonzentration $(3-4 \mathrm{n})$ in der Waschflüssigkeit entgegengewirkt. In der zweiten Extraktionsapparatur HS (Hot-Scrub-Extraktor) wird die Wäsche mit geringerer Salpetersäurekonzentration und $60^{\circ} \mathrm{C}$ Betriebstemperatur fortgesetzt. Die geringere Salpetersäurekonzentration begünstigt die Rückextraktion von Zirkon und Niob. Im selben Sinne wirkt die erhöhte Temperatur auf das Ruthen. Der nachfolgende Trennextraktor B arbeitet ebenfalls bei erhöhter Temperatur. Dadurch läßt sich die Plutonium-Konzentration in der wäßrigen Phase erhöhen und der Dekontaminationsfaktor des organischen Uran-Stromes auch im Falle eines Aktivitätsdurchbruches im HA Extraktor sicherstellen. Der wäßrige Plutoniumstrom wird anschließend im BS Extraktor (Scrub 
des B-Extraktors) bei erhöhter Temperatur von mitgenommenen Uranresten befreit. Im C-Extraktor erfolgt, ebenfalls bei höherer Temperatur zur Gewinnung höherer Konzentrationen, schließlich die Rückextraktion des Urans.

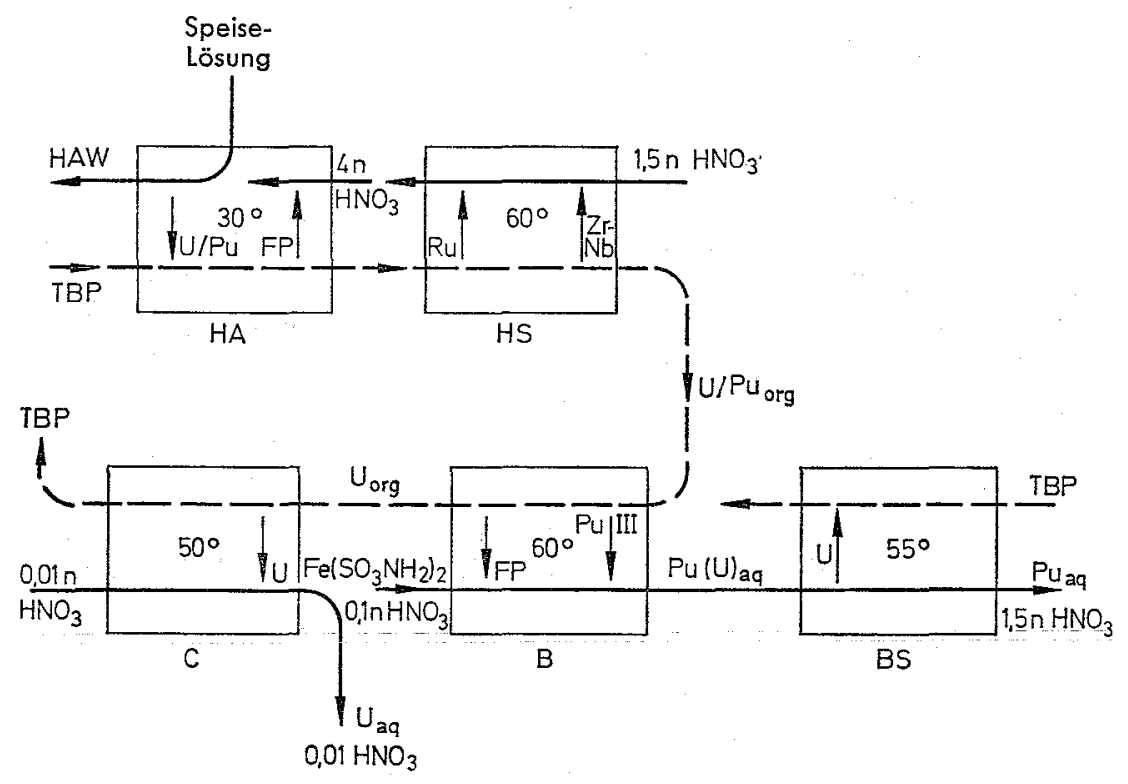

Abb. 7. Schema des Trenncyclus bei erhöhter Extraktionstemperatur. [HAW = hochaktiver Abfall (Waste), HA = Hot (hochaktiver) Extraktor A, HS = HotScrub Extraktor-(Wäscher), B = Extraktor B, BS = Scrub Extraktor B, C = Extraktor $\mathrm{C}, \mathrm{FP}=$ Spaltprodukte (Fission Products)]

Ein Vergleich der erzielbaren Dekontaminationsfaktoren zeigt, daß ein Trenncyclus mit erhöhter Temperatur für sich allein und nur mit etwa $2 / 3$ des apparativen Aufwandes ähnliche Werte erzielt wie ein 2-Cyclenprozeß (Trenncyclus + Reinigungscyclus) bei Normaltemperatur 52):

\begin{tabular}{lcc}
\hline & $D F-\mathrm{U}$ & $D F-\mathrm{Pu}$ \\
\hline 1 Cyclus bei erhöhter Temperatur & $10^{6}$ & $3 \cdot 10^{5}$ \\
\hline 2 Cyclen bei Normaltemperatur & $4 \cdot 10^{5}$ & $2 \cdot 10^{6}$ \\
\hline
\end{tabular}




\section{F. Baumgärtner und H. Philipp}

\section{Zukünftige Verfahren}

In den Forschungslaboratorien fast aller an der Entwicklung der Kernenergie beteiligten Länder werden seit Jahren mehr oder weniger intensive Versuche unternommen, neue Verfahren zur Wiederaufarbeitung von Kernbrennstoffen zu entwickeln. Dabei wird versucht, Verfahren zu finden, die gegenüber dem Purex-Prozeß günstiger sind, sowohl im apparativen Aufwand als auch hinsichtlich der Wirtschaftlichkeit des Gesamtprozesses.

\section{a) Bessere Extraktionsmittel}

Die bescheideneren Versuche zielen darauf ab, das bisherige Extraktionsmittel TBP durch ein besseres zu ersetzen. Seine allmähliche Zersetzung zu Dibutylphosphat (HDBP), Monobutylphosphat $\left(\mathrm{H}_{2} \mathrm{MBP}\right)$ und Phosphat ist unter dem Einfluß hoher Strahlungsdosen zweifellos ein Nachteil des TBP, weil diese Zersetzungsprodukte starke Komplexbildner für Uran und Plutonium sind und zu Produktverlusten führen 54). Darüber hinaus wirkt HDBP stark extrahierend auf Zirkon und verschlechtert dadurch den Dekontaminationsfaktor 36,55,56). Außerdem bildet $\mathrm{H}_{2} \mathrm{MBP}$ und $\mathrm{H}_{3} \mathrm{PO}_{4}$ mit Zirkon schwerlösliche Niederschläge, die sich in der Phasengrenzfläche abscheiden und sowohl den Dekontaminationsfaktor verschlechtern als auch zu erhöhter Radiolyse des Extraktionsmittels Anlaß geben 57).

Diese Schwächen sind jedoch nicht so gravierend, daß die Anwendung des TBP dadurch grundsätzlich infrage gestellt wäre ${ }^{58)}$.

Ein besseres Extraktionsmittel als TBP müßte nicht nur frei von ungünstigen Radiolyseprodukten sein, sondern es mäßte dazu auch die so vorteilhaften Eigenschaften des TBP wieder besitzen, nämlich die hohe Stabilität gegenüber konzentrierter Salpetersäure, die starke Aussalzwirkung, einen verschwindend geringen Dampfdruck, eine sehr geringe Löslichkeit in Wasser und ein ausgezeichnet hydrodynamisches Verhalten 59). Zweifellos gibt es Extraktionsmittel, die bessere Verteilungsfalktoren für Uran und Plutonium aufweisen als das TBP. Hohe Verteilungskoeffizienten sind für den technischen Einsatz jedoch nicht das Entscheidende, weil bei einem multiplikativen Verteilungsverfahren, das in der Regel angewendet wird, bereits Verteilungsfaktoren zwischen 2 und 10 genügen, um eine praktisch $100 \%$ ige Extraktionsausbeute zu erzielen.

Aber auch dann, wenn ein in allen Punkten besseres Extraktionsmittel gefunden sein sollte, entscheiden über den technischen Einsatz schließlich die wirtschaftlichen Gesichtspunkte. Ein charakteristisches Beispiel dafür ist die englische Wiederaufarbeitungsanlage Windscale-II 27). Obwohl die Anlage Windscale-I mit Butex als Extraktionsmittel durchaus befriedigend gearbeitet hatte (bis auf die ungünstige Ruthen-Dekontamination), mußte aus Kostengründen das Butex dem TBP in der zweiten Anlage weichen 27). Der Bedarf an Extraktionsmittel ist auch in einer technischen Wiederaufarbeitungsanlage nicht so groß, daß eine eigene Produktionsanlage für das Extraktionsmittel rentabel wäre. Aus diesem Grunde wird eine wirtschaftliche Wiederaufarbeitung auch in weiterer Zukunft auf ein einge- 
führtes, kommerzielles Produkt zurückgreifen müssen, das primär wahrscheinlich anderen $Z$ wecken dient als der Extraktion.

\section{b) Unkonventionelle Prozesse und das ,Aqua-Fluor-Verfahren“}

Die Untersuchungen über Wiederaufarbeitungsverfahren, die den wäßrigen Extraktionsprozeß durch ein anderes, nicht wäßriges Trennprinzip ersetzen sollen, konzentrieren sich besonders auf zwei Richtungen, nämlich auf Verflüchtigung der Fluoride von Uran und Plutonium und auf die pyrochemische Reinigung über Salz- oder Metallschmelzen.

Beide Methoden sind schon sehr weit ausgearbeitet. Die Literatur über beide Verfahren ist umfangreich, wie die Übersichtsartikel ${ }^{60-70)}$ zeigen. Dadurch aber, daß in der Technik kein $Z$ wang besteht, das bisherige wäßrige Verfahren aufzugeben, sind diese neuen, nichtwäßrigen Verfahren von vornherein einem Wirtschaftlichkeits-Vergleich mit dem eingeführten, wäßrigen Prozeß unterworfen. Vor einer Übernahme in die Technik müssen sie deshalb zumindest dieselbe Wirtschaftlichkeit und Betriebssicherheit garantieren wie das konventionelle Verfahren. Bei den unkonventionellen Methoden werden aber, wie es der Name schon sagt, zum Teil neuartige Techniken auf hochradioaktive Stoffe angewendet, die in ihrer Handhabung eine größere Betriebssicherheit erfordern als irgendwelche anderen Stoffe. Aus Sicherheitsgründen wird den unkonventionellen Prozessen von seiten der Techniker und Unternehmer deshalb schon mehr Zurückhaltung entgegengebracht, als es bei konventionellen Produktionsverfahren sonst der Fall wäre. Diese Umstände erschweren es jedem neuartigen Verfahren der Wiederaufarbeitung mehr, als es sonst vielleicht üblich ist, in der Industrie Fuß zu fassen.

Unter den ,unkonventionellen Methoden" der Wiederaufarbeitung ist der von General Electric projektierte „Aqua-Fhor-Prozeß" das ,,fortschrittlichste" Verfahren, das in der Technik zur Verwirklichung kommen soll ${ }^{71}$ ) (Abb. 8). Dieses Verfahren besitzt jedoch im ersten Verfahrensschritt ebenfalls einen Purex-Kodekontaminationscyclus. Daran schließt, nach Konzentrierung der Lösung und Einstellung auf $7 \mathrm{n} \mathrm{HNO}_{3}$, eine Plutonium-Abtrennung mittels Anionenaustauscher an. Das Plutonium-Eluat wird durch Eindampfen noch auf $250 \mathrm{~g} \mathrm{Pu} / 1$ konzentriert. Die Prozeßlösung wird dann reduziert, um Neptunium(IV) einzustellen,

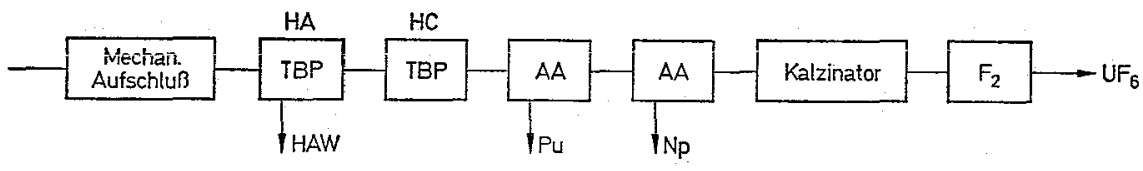

Abb. 8. Prinzipschema des Aqua-Fluor-Prozesses 71). Abkürzungen siehe Abb. 7 und Tabelle 2 
dessen Isolierung über einen anschließenden Anionenaustauscher folgt. Anschließend wird die Uran-haltige Prozeßlösung eingedampft, dann in einem Fließbett bei $350^{\circ} \mathrm{C}$ mit Heißdampf $\mathrm{zu} \mathrm{UO}_{3}$ calciniert. In einem Fluorinator folgt bei 350 bis $400^{\circ} \mathrm{C}$ mit elementarem Fluor die Umwandlung des Uranoxids in flüchtiges $\mathrm{UF}_{6}$. Zur weiteren Reinigung wird das Uranhexafluorid noch über eine NaF-Adsorptionssäule, dann durch eine einstufige Destillation und über ein $\mathrm{MgF}_{2}$-Adsorptionsfilter schließlich der fraktionierten Destillation zugeführt.

Also erst nachdem sowohl die Hauptmenge der Spaltprodukte als auch das Plutonium und Neptunium abgetrennt sind, folgt die „,fortschrittliche " Fluorid-Verflüchtigung des Urans mit entsprechenden Reinigungsschritten für das Uranhexafluorid.

Bemerkenswert sind die Gesichtspunkte, die zum Fließbild des Aqua-Fluor-Prozesses in dieser Form geführt haben ${ }^{22)}$. Hier zeigen sich besonders deutlich die Voraussetzungen, die ein neues Verfahren zu erfüllen hat, noch ehe über seine Wirtschaftlichkeit im Detail diskutiert wird:

1. Die Möglichkeit der zuverlässigen Analysennahme für eine genaue Durchsatzkontrolle und Bilanzierung des eingebrachten Spaltstoffs und zur genauen Feststellung des Abbrandes. (Beide Größen, Spaltstoffgehalt und Abbrand, stellen die Abrechnungsbasis bei der Wiederaufarbeitung dar.)

2. Vollständige Rückgewinnung $(>98 \%)$ des Plutoniums, Urans und Neptuniums aufgrund des hohen Materialwertes dieser Stoffe.

3. Erhaltung der Isotopenzusammensetzung des Materials (betrifft ebenfalls den Wert des Urans und Plutoniums).

4. Zuverlässiger zeitlicher Betriebsablauf aus Gründen der Wirtschaftlichkeit des gesamten Brennstoffkreislaufes.

Diese Grundvoraussetzung für eine wirtschaftliche Wiederaufarbeitung konnte ein Gesamt-Fluorierungs-Prozeß nicht erfüllen, sondern erst der wesentlich abgewandelte Aqua-Fluor-Prozeß 72).

\section{Entwicklungsprobleme der wäßrigen Wiederaufarbeitung}

Die zukünftigen Aufgaben der Wiederaufarbeitung von Uran-Plutonium-Brennstoffen werden bestimmt von der Entwicklung der Kraftwerksreaktoren. Die Probleme lassen sich dabei in folgende zwei Gruppen klassifizieren:

1. Die zukünftige, chemische Form der Kernbrennstoffe (PlutoniumGehalt, Oxide, Carbide etc.).

2. Der zukünftige Spaltprodukt-Gehalt aufgrund der Leistungsdichte, des nuklearen Abbrandes und der Kühlzeit der Brennstäbe. 
1. Die chemische Form des Brennstoffs bei zukünftigen Uran-PlutoniumReaktoren

Die Entwicklung tendiert einerseits zu Brennelementen mit höherem Plutoniumgehalt und andererseits von den jetzigen Oxiden zu den Carbiden. Der höhere Plutoniumgehalt hat zwei Motive: in LeichtwasserReaktoren kann das Uran-235 durch Kraftwerksplutonium ersetzt werden. Das führt zu Brennelementen mit einem Gehalt von einigen Prozenten an Plutonium.

Bei Schnellen Brutreaktoren wird das Plutonium zu einer grundsätzlichen Notwendigkeit. Der Plutonium-Gehalt wird dort zwischen 10\% und $20 \%$ liegen.

Der Grund für das Bestreben, von den Oxiden des Urans und Plutoniums zu den Carbiden überzugehen, liegt in der höheren Wärmeleitfähigkeit der Carbide. Bei erhöhter Wärmeleitfähigkeit läßt sich die Leistungsdichte im Brennstab steigern und damit auch die Wirtschaftlichkeit der Energiegewinnung. Der unmittelbaren Verwendung der Carbide stehen die geringen Homogenitätsbreiten im Phasendiagramm entgegen, die bei höherem Abbrand Phasenumwandlungen und Kohlenstoffausscheidungen zur Folge hätten. Weiterhin bringen die Carbide durch die Möglichkeit einer chemischen Reaktion mit dem Kühlmittel ein erhöhtes Betriebsrisiko im Falle-eines defekten Brennelementes mit sich.

\section{a) Der erhöhte Plutonium-Gehalt}

๙) Die Brennstoff-Auflösung

$\mathrm{PuO}_{2}$ ist in verdünnter und auch konzentrierter Salpetersäure unlöslich. Durch Zugabe von Fluorid als Komplexbildner kann eine Auflösung erreicht werden. Im technischen Betrieb ist die Verwendung von Fluorid wegen der erhöhten Korrosion jedoch nicht erwünscht. Um rostfreien Stahl als Werkstoff verwenden zu können, ist reine Salpetersäure notwendig. Untersuchungen an unbestrahlten $\mathrm{PuO}_{2} / \mathrm{UO}_{2}$-Proben haben gezeigt 73,74), daß eine relativ gute Auflösung des Plutoniumoxids erreicht werden kann, wenn durch eine vorausgegangene Sinterung das Gemenge aus $\mathrm{PuO}_{2}$ und $\mathrm{UO}_{2}$ in eine homogene Mischkristallphase überführt worden ist. Bei bestrahlten Proben soll, offenbar durch die erhebliche Störung der Gitterstrulutur mit einer erhöhten Löslichkeit des Plutoniumoxids in Salpetersäure zu rechnen sein ${ }^{75}$ ).

\section{B) Die Extraktion}

Auch bei der Extraktion ergeben sich durch den Verdrängungseffekt des Urans (vgl. Abb. 3, II/2) mit steigender Plutoniumkonzentration zusätzliche Schwierigkeiten 76,77). Bei einer einstufigen Extraktion der 
Uran-Plutonium-Lösung würde der Verdrängungseffekt zu einer ungenügenden Plutonium-Extraktion und damit $\mathrm{zu}$ einem Verlust an Plutonium führen. Bei der technischen Mehrstufenextraktion wird die Speiselösung dem Mischabsetzer oder der Extraktionskolonne ungefähr in der Mitte zugeführt (vgl. Abb. 9). Durch den Verdrängungseffekt nimmt das Plutonium am Speisepunkt aber nicht in vollem Maße an der Extraktion teil, sondern wird mit der wäßrigen Phase zum Uran-ärmeren Teil des Extraktionsapparates geführt, um dort in die noch wenig beladene, organische Phase überzutreten. Wenn diese organische Plutonium-Lösung dann durch das Gegenstromverfahren den Speisepunkt erreicht, wird das Plutonium durch das Uran wieder in die wäßrige Phase zurückgedrängt und wandert mit dem frisch hinzugekommenen Plutonium erneut in den Uran-ärmeren Teil der Extraktionsanlage. Durch diesen Kreislauf konzentriert sich das Plutonium in einem Teil der Extraktionsanlage auf und kann ein nukleares Kritikalitätsrisiko hervorrufen, Abb. 9 78), (vgl. auch IV, 1a, $\delta$ ).

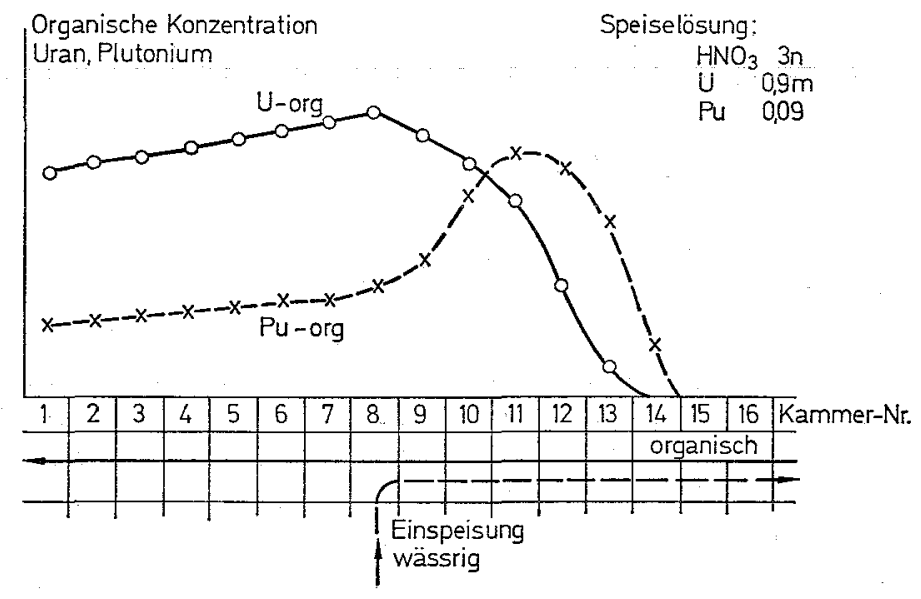

Abb. 9. Verteilungsprofil des Plutoniums und Urans bei der Ko-Extraktion in einem multiplikativen Verteilungsverfahren

Diese Gefahr besteht besonders im ersten Extraktionsapparat der Wiederaufarbeitungsanlage, weil hier noch der ungeteilte Prozeßstrom einen hohen Durchsatz erfordert und der Apparat aus diesem Grunde in der Regel nicht kritikalitätssicher dimensioniert ist. 
Die Wiederaufarbeitung von Uran-Plutonium-Kernbrennstoffen

\section{Ү) Die Uran-Plutonium-Trennung}

Die extraktive Uran-Plutonium-Trennung gelingt durch Reduktion des Plutonium(IV) und -(VI) zu Plutonium(III) mittels Fe ${ }^{2+}$ oder $\mathrm{U}^{4+}$. Wie in Abschnitt III/3 eriäutert worden ist, braucht eine vollständige Plutoniumreduktion einen mehrfach äquivalenten Überschuß des Reduktionsmittels. Ein Anstieg des Plutonium-Gehaltes auf 10-20\%, wie es beim Schnellen Brüter geplant ist, würde dementsprechend einen Bedarf an Reduktionsmitteln erfordern, der in der Größenordnung des Produktstromes liegt. Aus diesem Grunde kann Eisen(II) zumindest für Brüter-Brennstoff nicht mehr verwendet werden, wenn nicht eine erhebliche Überdimensionierung der entsprechenden Anlageteile, eine zusätzliche Plutonium-Eisen-Trennung und eine aufwendige Lagerung von Plutonium-kontaminiertem Eisen in Kauf genommen werden soll. Aber auch Uran(IV) ist wegen der großen erforderlichen Mengen kein ideales Reduktionsmittel mehr. Es hat deshalb nicht an Versuchen gefehlt, dieses Problem ohne substantiell ins Gewicht fallende Reduktionsmittel $z u$ lösen. In Oak Ridge wurde dazu eine katalytische Reduktion des Plutoniums in der organischen Phase mit Wasserstoff-Gas und einem Platinkatalysator entwickelt 79). In Karlsruhe gelang im Labormaßstab die elektrolytische Reduktion des Plutoniums aus der organischen Phase.

\section{ס) Kritikalität}

Ein erhöhter Plutoniumgehalt im Brennstoff bringt das Problem der nuklearen Kritikalität stärker in den Vordergrund, als bei der Wiederaufarbeitung von reinen Uran-Brennstoffen. Das liegt darin begründet, $\mathrm{da} ß$ in wäßriger Lösung oder Suspension die minimal kritische Masse für Plutonium mit $520 \mathrm{Gramm}$ beträchtlich geringer ist als die von Uran-235, die 820 Gramm beträgt. Dazu kommt, daß bei einer Ausfällung des Plutoniums weitgehend das reine spaltbare Material angehäuft wird, während beim Uran eine Verdünnung durch Uran-238 um mehr als den Faktor zwanzig besteht. Darüber hinaus erfolgt beim Plutonium aus chemischen Gründen eine Materialanhäufung leichter als beim Uran: z.B. durch ungelöstes $\mathrm{PuO}_{2}$ bei der Auflösung 73), bei Säurekonzentrationen unter 1,5 n durch Ausfällung von Plutonium-hydroxid (,Polymer ${ }^{\prime \prime}{ }^{81)}$, während des Extraktionsvorganges durch Rezirkulation ${ }^{78)}$ oder als Niederschlag mit den Abbauprodukten des TBP 82).

Grundsätzlich gibt es drei Möglichkeiten, die Kritikalitätssicherheit in einer chemischen Anlage zu gewährleisten: Begrenzung der Masse des spaltbaren Materials oder des Volumens, das die spaltbare Masse einnimmt, Begrenzung der Konzentration des spaltbaren Materials und schließlich Begrenzung der Apparatedimension, in der das spaltbare Material verarbeitet wird (geometrische Kontrolle). Wegen des erforderlichen Durch- 
satzes scheidet bei technischen Anlagen die Massenkontrolle und Volumenkontrolle aus. Bei den bisher gebräuchlichen Extraktionsapparaturen (Mischabsetzern und Extraktionskolonnen) läßt sich auch die geometrische Kontrolle nicht ohne erhebliche Kapazitätsbeschränkung einführen: Für Plutonium-,,Polymer" (Hydrolysat) beträgt die minimal kritische Schichtdicke 4,4 cm und der minimal kritische Zylinderdurchmesser 13,1 cm. Für geometrisch sichere Apparate sind 10\% bis 30\% kleinere Dimensionen zu wählen, so daß geometrisch sichere Anlagen nur Apparate und Behälter mit verhältnismäßig kleiner Dimension besitzen. In technischen Anlagen ist es üblich, nukleare Kritikalität durch Ausüben von Konzentrationskontrollen zu verhindern ${ }^{83)}$. Dazu sind allerdings sehr zuverlässige und strenge Kontrollen über alle Prozeßströme erforderlich 84,85). Zusammenfassende Darstellungen über das Problem der nuclearen Kritikalität in chemischen Anlagen sind an anderer Stelle gegeben $86,87,88)$.

\section{b) Carbidische Brennstoffe}

Obwohl sich die Uran- und Plutoniumkarbide und auch die Mischcarbide beider Elemente vollständig in 2 bis 16 normaler Salpetersäure auflösen, ist dieser Auflösevorgang nicht als Vorstufe für den Purex-Prozeß anwendbar 89,90). Sowohl an unbestrahlten als bestrahlten Proben zeigte sich, daß 30-50\% des Carbidkohlenstoffs zu organischen Säuren (Oxalsäure, Melitsäure usw.) hydrolysiert, wodurch der anschließende Extraktionsproze $B$ entweder durch Emulsionsbildung oder durch Komplexbildung in der organischen Phase gestört wird. Aus diesem Grunde muß zumindest ein Oxidationsschritt in Form von längerem Kochen der Lösung mit Salpetersäure oder $\mathrm{KMnO}_{4}$ dem Extraktionsvorgang vorausgehen.

Eine Variante des einfachen Lösungsvorgangs ist die normale Hydrolyse bei $100^{\circ} \mathrm{C}$ mit anschließender Auflösung der Oxide in Salpetersäure.

Dabei treten jedoch durch die Hydrolyseprodukte des Carbid-Kohlenstoffs dieselben Schwierigkeiten auf, wie beim normalen Löseprozeß in Salpetersäure. Bestrahlte Carbide zeigten sogar eine Passivierung gegenüber der $100^{\circ}$ Hydrolyse ${ }^{89}$, so daß dieses Verfahren kaum zu einer technischen Anwendung kommen wird.

Mit gutem Erfolg ist bisher die Pyro-Hydrolyse mit Heißdampf von $750^{\circ} \mathrm{C}$ zum AufschluB der Carbide angewendet worden. Dabei entstehen $\mathrm{H}_{2}, \mathrm{CO}_{2}$ und $\mathrm{CO}$ als gasförmige Reaktionsprodukte, die bei bestrahlten Kernbrennstoffen auch die Spalt-Edelgase und einen Teil des Spalt-Jods mitführen ${ }^{90}$. Bei der anschließenden Extraktion der aufgelösten Oxide soll der störende Einfluß des Restkohlenstoffs in Form organischer Reaktionsprodukte nur geringfügig sein. Das Verfahren wurde sowohl an bestrahlten wie unbestrahlten Proben mit Erfolg getestet ${ }^{89)}$. Es bleibt 
jedoch die Frage offen, ob das oxidische Hydrolyse-Produkt des Plutoniums auch in technischem Maßstab in jedem Fall zuverlässig in $\mathrm{HNO}_{3}$ lösbar ist.

Weiterhin besteht noch die Möglichkeit des oxidierenden Aufschlusses von Carbiden mit Luft oder Luft-Sauerstoff bei erhöhter Temperatur. Über die technische Verwendbarkeit dieses Verfahrens ist jedoch noch weniger bakannt ${ }^{91)}$.

Wenn die Carbide einmal in Salpetersäure gelöst und die Hydrolyseprodukte des Kohlenstoffs eliminiert worden sind, ergeben sich für den weiteren Prozeßablauf keine zusätzlichen Schwierigkeiten mehr.

\section{Abbrand, spezifische Stableistung und Kühlzeit zukünftiger Brennstäbe}

Von den technischen Reaktordaten bestimmt die spezifische Leistung während des Reaktorbetriebes $(\mathrm{kW} / \mathrm{kg}$ ) und der Abbrand des spaltbaren Materials, die Konzentration und Radioaktivität der Spaltprodukte bei der Wiederaufarbeitung. Die spezifische Stableistung ist ein Maß für die räumliche Dichte, mit der die Spaltprodukte erzeugt werden; der $\mathrm{Ab}$ brand ist ein $\mathrm{Maß}$ für die Gesamtmenge der vorliegenden Spaltprodukte. Die gebräuchliche Dimension für den Abbrand ist $1 \mathrm{MWd}$ pro kg oder pro Tonne (1 MWd entspricht der Spaltung von ca. $1 \mathrm{~g} \mathrm{U}-235$, (genau 1,054 g) d.h. $10 \mathrm{MWd} / \mathrm{kg}$ ist äquivalent ca. $1 \%$ Abbrand).

Konventionelle Siede- und Druckwasserreaktoren arbeiten heute bei spezifischen Leistungen von 15 und $25 \mathrm{~kW} / \mathrm{kg}(\mathrm{U}+\mathrm{Pu})$ und mit Abbränden von 20-25 MWd/kg ${ }^{3)}$. Im Bestreben, die Wirtschaftlichkeit zu steigern, dürfte die Entwicklung bei Leichtwasserreaktoren auf spezifische Leistungen von $30-40 \mathrm{~kW} / \mathrm{kg}$ und auf Abbrände von $30-40 \mathrm{MWd} / \mathrm{kg}$ ansteigen ${ }^{92)}$.

Bei den zukünftigen Schnellen Brutreaktoren, so ist heute die Ansicht, sollen die Brennelemente des Reaktorkerns und des radialen Brutmantels gemeinsam aufgearbeitet werden 83,93). Die über beide Brennelementtypen gemittelten Abbrände und spezifische Stableistungen liegen in derselben Größenordnung wie die genannten Werte für die zukünftigen Leichtwasserreaktoren 3). Für eine realistische Beurteilung der entstehenden Schwierigkeiten sind jedoch die Brennelementeinheiten, d.h. die Verhältnisse bei einem zentralen Brennstab in Rechnung zu setzen. Der Abbrand des Schnellen Bruters soll maximal 100 bis $110 \mathrm{MWd} / \mathrm{kg}$, im Mittel über die gesamte Kernzone ca. $80 \mathrm{MWd} / \mathrm{kg}$ erreichen. Ein zentrales Brennelement enthält jedoch nicht nur das Material des Reaktorkerns, sondern $2 / 3$ des Brennstabes am oberen und unteren Ende haben Anteil an der Mantelzone, die durch einen Abbrand von nur rund $5 \mathrm{MWd} / \mathrm{kg}$ gekennzeichnet ist. Als mittleren Abbrand über den gesamten Stab ergeben sich damit 50-55 MWd $/ \mathrm{kg}$, d.h. 5 bis $6 \%$ Abbrand innerhalb eines zentralen 
Stabes und eine mittlere spezifische Leistung zur Zeit der Entnahme des Brennelements von 100 bis $120 \mathrm{~kW} / \mathrm{kg}$. Der daraus resultierende, verhältnismäßig hohe Gehalt an radioaktiven Spaltprodukten hat verschiedenartige Folgen, die sich beim Transport, beim Aufschlußverfahren und beim Extraktionsprozeß auswirken.

\section{a) Nachwërmeleistung}

Der radioaktive Zerfall der Spaltprodukte hat eine Wärmeentwicklung im Brennstab zur Folge. Bei thermischen Reaktoren liegt diese Nachwärmeleistung in der Größenordnung von $10 \mathrm{~W} / \mathrm{kg}$ Brennstoff und bedarf noch nicht der künstlichen Kühlung. Bei den Schnellen Brutreaktoren jedoch steigt die Nachwärmeleistung lokal im Stabbereich des Reaktorkerns auf 100-200 W/g an. Diese Wärmeentwicklung ist so hoch, daß eine künstliche Kühlung notwendig wird, und zwar sowohl während der Lagerung und dem Transport, als möglicherweise auch während der Brennelementzerlegung ${ }^{94)}$.

\section{b) Der Löseproze $\beta$}

Beim Auflösevorgang ist allein schon durch die Selbsterhitzung aufgrund der radioaktiven Zerfallswärme, eine gesteigerte Auflösegeschwindigkeit zu erwarten. Der dadurch rasch anschwellende Auflösevorgang wurde schon ab $0,5 \%$ Abbrand beobachtet ${ }^{75}$ ). Problematisch kann in diesem $\mathrm{Zu}-$ sammenhang die rasche Freisetzung der radioaktiven Spaltgase werden, weil dadurch ein entsprechend wirksameres Abgasreinigungssystem erforderlich ist.

Als eine unerwartete Folge des hohen Abbrandes wurde beim Auflösen oxidischer Brennelemente ein feiner metallischer Rückstand gefunden, der aus einer Legierung der Spaltelemente Molybdän, Zirkon, Ruthen, Palladium und Technetium besteht und auch geringe Mengen Uran und Plutonium enthält 95). Die Ursache dieser selektiven Abscheidung einzelner Spaltprodukte in metallischer Form ist noch ungeklärt. Möglicherweise findet die Anreicherung während des Reaktorbetriebes in einer Art Transportreaktion unter Mitwirkung des Spaltjods statt. Die hohe spezifische Aktivität (ca. $1500 \mathrm{Ci} / \mathrm{g}$ ) und Wärmeabgabe (ca. $4 \mathrm{~W} / \mathrm{g}$ ) erfordert die Abtrennung dieser Partikel vor dem Extraktionsprozeß.

\section{c) Der Extraktionsprozeß}

Die Erhöhung der Stableistung oder des Abbrandes wirken sich nicht im gleichen Maße auf den erforderlichen Dekontaminationsfaktor aus. Die Spaltproduktaktivität steigt bei Erhöhung der Leistungsdichte stärker an als bei Spaltungs-äquivalenter Zunahme des Abbrandes. Bei höherer Leistungsdichte wird bei gleicher Gesamtzahl an Spaltungen 
die Bestrahlungszeit entsprechend kürzer. Dadurch tragen früher gebildete und noch nicht zerfallene Spaltprodukte einen zusätzlichen Teil bei zur Gesamtaktivität. Abb. 10 gibt die notwendigen Dekontaminationsfaktoren in Abhängigkeit verschiedener Leistungsdichten und verschiedener Abbrände bei 30 und 100 Tagen Abklingzeit wieder. Diese Angaben beruhen auf berechneten Spaltprodukt-Aktivitäten ${ }^{96}$ ) der thermischen Uran-235 Spaltung. Als Ziel der Dekontamination wurde dabei die $\beta$-Aktivität des natürlichen Urans im Gleichgewicht mit seinen kurzlebigen Folgeprodukten $\mathrm{UX}_{1}, \mathrm{UX}_{2}, \mathrm{UZ}$ und $\mathrm{UY}$ von $0,6 \cdot 10^{-6} \beta-\mathrm{Ci} / \mathrm{g}$ angenommen.

Eine strenge Berechnung des Dekontaminationsfaktors, der mit einem gewählten Extraktionsschema zu erreichen ist, kann nicht durchgeführt werden, weil die Ionen der Spaltnuklide Ruthen, Zirkon und Niob während des Extraktionsvorganges chemische Umwandlungen erfahren, wodurch sich ihre Extraktionseigenschaften ändern. Es kann aber ein Vergleich gezogen werden mit Angaben, die in der Praxis gewonnen worden sind. Tabelle 3 zeigt die erreichten Dekontaminationsfaktoren bei verschiedenen Cyclenkombinationen im Purex-Prozeß.

Aufgrund dieser allgemeinen Daten läßt sich damit rechnen, daß ein Purex-Prozeß mit 3 Cyclen oder mit 2 Cyclen und erhöhter Arbeitstemperatur auch die in Zukunft anfallenden Brennelemente befriedigend kontaminieren wird.

Bei derAufarbeitung von Materialprüf-Reaktoren sind wegen der hohen spezifischen Leistung von mehreren tausend $\mathrm{kW} / \mathrm{kg}$ und Abbränden von

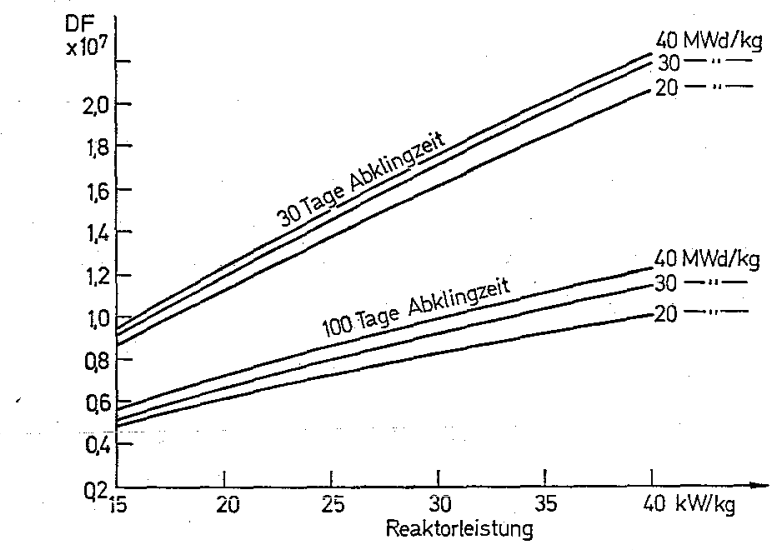

Abb. 10. Notwendige Dekontaminationsfaktoren für Uran und Plutonium gegenüber natürlichem Uran $\left(0,67 \cdot 10^{-3} \mathrm{Ci} / \mathrm{kg}\right)$ in Abhängigkeit von Reaktorleistung und $\mathrm{Ab}$ brand für 30 und 100 Tage Abklingzeit. Berechnet auf Grund der Aktivitätsangaben von Blomeke und Tòdd 96) 
F. Baumgärtner und H. Philipp

\begin{tabular}{lll}
$\begin{array}{l}\text { Tabelle 3. Dekontaminationsfahtoren beim } \\
\text { nach verschiedenen }\end{array}$ & Prbeitsverfahren & 52,94) \\
\hline Purex-ProzeB & Uran & PF \\
& DF & DF \\
\hline 3 Cyclen & $1,6 \cdot 10^{7}$ & $4 \cdot 10^{7}$ \\
\hline $\begin{array}{l}2 \text { Cyclen } \\
+ \text { Silikagel und } \\
\text { Anionenaustauscher }\end{array}$ & $4 \cdot 10^{6}$ & $3,2 \cdot 10^{7}$ \\
\hline $\begin{array}{l}\text { 1 Cyclus } \\
\text { bei erhöhter Temperatur } \\
+ \text { Anionenaustauscher } \\
\text { und Silikagel }\end{array}$ & $10^{6}$ & $3 \cdot 10^{5}$ \\
\hline
\end{tabular}

hundert MWd $/ \mathrm{kg}$ und mehr schon heute Dekontaminationsfaktoren von $10^{8}$ notwendig. In einem Zwei-Cyclen-Prozeß mit erhöhter Betriebstemperatur soll für diesen Fall auch ein Dekontaminationsfaktor von $2 \cdot 10^{8} \mathrm{zu}$ erreichen sein ${ }^{98}$ ). Allerdings ist dazu im ersten Extraktor ein Säureunterschuß notwendig, um die Zirkon-Niob-Extraktion von vornherein zu unterdrückeñ. Beì einem höheren Plutoniumgehalt wäre diese Arbeitsweise wegen der Hydrolyse des Plutoniums nicht anwendbar. Andererseits kann daraus aber geschlossen werden, daB bei der Wiederaufarbeitung schneller Brutreaktoren, wenn DF-Faktoren von $10^{8} \mathrm{er}-$ forderlich werden, grundsätzlich doch die Möglichkeit besteht, durch Kombination eines Zwei-Cyclen-Prozesses mit erhöhter Betriebstemperatur und Vorschaltung eines Kodekontaminationscyclus diese Werte zu erreichen. Schwierigkeiten werden allerdings die Spaltelemente ZirkonNiob und vor allem Ruthen bereiten, dessen Spaltausbeute bei Plutonium-239 mit schnellen Neutronen von 0,38 auf 6,35\% steigt ${ }^{99}$ ). Die Komplexchemie und das Extraktionsverhalten dieser Elemente ist deshalb ein aktuelles und technisch wichtiges Forschungsgebiet im Zusammenhang mit der Wiederaufarbeitung.

\section{d) Das Radiolyseproblem}

Die mit steigendem Abbrand erforderlichen Dekikontaminätionsfaktoren dürfen jedoch nicht nur von der Leistungsfähigkeit eines Fließbildes her diskutiert werden. Einen besonderen Faktor nimmt dabei die mit steigender Spaltproduktaktivität ansteigende, radiolytische Zersetzung des Lösungsmittels ein 100).

Die Folgen der Lösungsmittelradiolyse lassen sich grob in ein temporäres und ein bleibendes Erscheinungsbild unterteilen. Die temporäre 
Erscheinung wird der TBP-Zersetzung zugeordnet, deren Produkte sich durch eine alkalische Lösungsmittelwäsche beseitigen lassen. Die Radiolyse des Verdünnungsmittels dagegen führt zu organischen StickstoffSauerstoff-Verbindungen, die nicht von der alkalischen Wäsche beseitigt werden und sich im Lösungsmittel aufbauen.

\section{a) Die TBP-Zersetzung}

Die TBP-Zersetzung geht stufenweise über $\mathrm{HDBP}$ und $\mathrm{H}_{2} \mathrm{MBP}$ zu $\mathrm{H}_{3} \mathrm{PO}_{4}$ vor sich. Den überwiegenden Teil der Zersetzungsprodukte stellt in der Regel das HDBP 101,102). Die Bildungsrate des Di-butylphosphats wird neben der ionisierenden Dosisleistung auch noch von der Salpetersäurekonzentration, dem Urangehalt und dem Verdünnungsmittel beeinflußt 103,104). Außerdem hat auch Sauerstoff einen starken Einfluß auf die HDBP-Bildung; damit ist auch der Einfluß der Rührintensität auf den Radiolysevorgang zu erklären 105).

Die schädliche Wirkung des HDBP beruht auf einer Komplexbildung mit Uran(VI), Plutonium(IV), Zirkon und Niob 36,55,56,57,101,106,107, 108). Die Komplexbildung mit Uran und Plutonium verhindert die Rückextraktion dieser Elemente, während die Komplexbildung mit Zirkon die Extraktion des Zirkons begünstigt und bei höheren Konzentrationen zur_Ausfällung führt 57,109). Die Reihenfolge der Komplexbildungsstärke 110) ist: $\mathrm{Zr}>\mathrm{Pu}(\mathrm{IV})>\mathrm{U}(\mathrm{VI})>\mathrm{Nb}$.

Das Ruthen soll durch HDBP nicht beeinflußt werden 111).

Trotz der stärkeren Komplexbildung des Zirkons und Plutoniums werden diese Elemente durch den Überschuß an Uran aus dem HDBPKomplex verdrängt, so daß als erste Folge der TBP-Radiolyse Uranverluste eintreten. Bei Brüter-Brennstoffen ist wegen des Plutoniumanteils von $10 \%-20 \%$ bei der zu erwartenden Strahlenbelastung von $1 \mathrm{Wh} / 1$ auch mit erheblichen Plutoniumverlusten zu rechnen.

Auch eine alkalische Lösungsmittelwäsche ist dann nicht in der Lage, diese Plutoniumkomplexe vollständig zu zersetzen ${ }^{105)}$. Eine zusätzliche Anwendung von Fluorid bei der alkalischen Wäsche ist dazu notwendig. Am besten sind die Plutoniumverluste mit einer reduzierenden Rückextraktion $\mathrm{zu}$ unterbinden. Auch bei hochplutoniumhaltigen Brüterbrennstoffen lassen sich die Plutoniumverluste dann auf weniger als $0,02 \%$ erniedrigen ${ }^{112}$.

Zur Verbesserung des Dekontaminationsfaktors gegenüber Zirkon ist naheliegend, der Prozeßlösung inaktives Zirkon zuzusetzen 101). Dieser Vorschlag hat in der Technik jedoch keinen Eingang gefunden.

Besonders störend für den Prozeßablauf sind die bei höheren Dosisleistungen auftretenden Zirkonausfällungen, die sich an der Phasengrenzfläche ansammeln. Es besteht der Vorschlag, diese Niederschläge durch eine dosierte Zugabe von Fluorid-Ionen zu verhindern 57). Proble- 
matisch dabei bleibt, wie die gleichzeitige Komplexierung des Plutoniums und die damit verbundenen Plutoniumverluste sicher vermieden werden.

\section{ß) Lösungsmittelzersetzung}

Bei den organischen Verdünnungsmitteln besteht hinsichtlich der thermischen und radiolytischen Zersetzung folgende Stabilitätsreihe: nParafine $>$ Iso-Paraffine $>$ Naphtene $>$ Olefine 104,113,114,115). Bemerkenswert ist die Erfahrung, daß die TBP-Zersetzung zunimmt mit steigender Stabilität des Verdünnungsmittels 104,110). Offenbar wirkt ein Energieleitungsmechanismus, der die Strahlungsenergie an der bindungsmäßig schwächsten Stelle des Systems zur Wirkung bringt. Aromatische Kohlenwasserstoffe wären deshalb als besonders geeignete Schutzreagenzien für das TBP anzusehen 116,119). Ihr Einsatz in technischen Anlagen wird jedoch wegen der Gefahr von Nitrierungsreaktionen vermieden.

Die Auswirkung der Lösungsmittelradiolyse besteht zum wesentlichen Teil in einer Verschlechterung des Dekontaminationsfaktors gegenüber Zirkon. Über den Einfluß auf das Spaltruthen besteht keine übereinstimmende Meinung 110,117,118).

Die primären radiolytischen Zersetzungsprodukte sind Nitro- und Carbonylverbindungen, Carbonsäuren, Nitratester etc. Keines dieser primären Oxidations- oder Nitrierungsprodukte zeigt jedoch die störende Komplexierungseigenschaft gegenüber Zirkon 113).

Auf die störende Wirkung durch ein Sekundärprodukt weist auch der zeitliche Bildungsablauf hin. Der Komplexbildner wächst nicht linear wie die Primärprodukte an, sondern mit einer zeitlichen Verzögerung 110). Am deutlichsten kommt die sekundäre Bildung in der Beobachtung zum Ausdruck, daß der Aufbau des Komplexbildners auch noch nach Beendigung der Bestrahlung weiterhin zunimmt 105). Auf diese Tatsache stützt sich die Hoffnung, den Entstehungsprozeß des störenden Komplexbildners eines Tages hemmend beeinflussen zu können.

Ưber die chemische Konstitution dieses unbekannten Radiolyseproduktes bestehen nur Vermutungen, die sich auf die Hydroxamsäurekonfiguration-COHNOH konzentrieren 113,114,115). Die sicheren experimentellen Belege dafür stehen jedoch noch aus.

Zur Beseitigung der Radiolyseprodukte wird in den technischen Anlagen das Lösungsmittel in der Regel einer alkalischen Wäsche $\left(\mathrm{Na}_{2} \mathrm{CO}_{3}\right.$, $\mathrm{NaOH})$ unterzogen. In einzelnen. Fällen sind auch oxidierende $\left(\mathrm{KMnO}_{4}\right)$ oder saure $\left(\mathrm{HNO}_{3}\right)$-Wäschen versucht worden ${ }^{110}$ ). In erster Linie werden dadurch aber nur die Zersetzungsprodukte des TBP erfaßt und die DBPKomplexe der Spaltprodukte zerlegt. Als besonders wirksame effektive Extraktionsmittel für die störenden Komplexbildner haben sich Alkanolamine erwiesen $113,115,117,120$ ). In der Technik sind sie jedoch aus Kostengründen bisher nicht eingesetzt worden. 
y) Die voraussichtliche Situation der Zukunft

Die radiolytische Zersetzung des organischen Extraktionsmittels ist lange Zeit als das schwerwiegendste Argument gegen eine Wiederaufarbeitung fortgeschrittener Kraftwerksreaktoren mit dem Purex-Prozeß angesehen worden. Zum Teil ging diese Meinung zurück auf die amerikanischen Erfahrungen bis 1958 121). Andere Untersuchungen bestätigten außerdem diese Meinung 122,123). Eine kritische Überprüfung dieser Ergebnisse in jüngster Zeit 58) hat jedoch gezeigt, daß den früheren Angaben teilweise eine Fehlinterpretation zugrunde lag und teilweise die experimentellen Dosen viel zu hoch gelegt worden waren, um sie mit den realen Gegebenheiten bei der Wiederaufarbeitung vergleichen zu können. Die maximale Dosisleistung wird in Zukunft voraussichtlich bei den Brennelementen der Schnellen Brutreaktoren auftreten. Die auf das Extraktionsmittel wirkende Dosisleistung wird dabei in der Größenordnung von wenigen $W h / 1$ liegen 58,83,124,125,126). Radioaktive Proben mit derartigen Dosisleistungen sind von TBP-Lösungen bisher schon mehrmals verarbeitet worden 117,127,128). Deshalb dürfte auch in $\mathrm{Zu}$ kunft die Radiolyse des Extraktionsmittels nicht mehr als der Engpaß in der Anwendung des Purex-Prozesses gesehen werden. Sollten sich trotzdem Schwierigkeiten einstellen, so besteht noch die apparative Möglichkeit, die Strahlenbelastung des Extraktionsmittels durch Verkürzung der Kontaktzeit wesentlich zu erniedrigen. Die Verweilzeit pro Extraktionsstufe in den herkömmlichen Mischabsetzern beträgt einige Minuten. In Schnellextraktoren wird diese Zeit auf wenige Sekunden verkürzt. Geeignete Schnellextraktoren wurden in den USA (SavannahRiver, Oak Ridge) und in Karlsruhe entwickelt und erprobt 129-136).

Die konventionellen Schnellextraktoren (Podbielniak, Luwesta) sind aus Gründen der Kritikalität in Wiederaufarbeitungsanlagen nicht zu verwenden.

\section{e) Das Abgasproblem}

Mit steigendem Abbrand und zunehmender Leistungsdichte der Reaktoren tritt das Problem der radioaktiven Abgase Krypton-85, Tritium und Jod-131 (sowie Xe-133 und Jod-129) in den Vordergrund.

\section{๔) Krypton-85 (Xe-133)}

In einem thermischen Reaktor werden $4450 \mathrm{Ci} \mathrm{Kr-85}$ erzeugt pro 10000 MWd Abbrand. Bei Schnellen Reaktoren entstehen wegen der geringeren Spaltausbeute ${ }^{99)}$ nur $1130 \mathrm{Ci} \mathrm{Kr-85}$ pro $10000 \mathrm{MWd}$. Dementsprechend fallen in einer Wiederaufarbeitungsanlage mit 1 Tonne Tageskapazität je nach Abbrand der Brennelemente Kr-85 Aktivitäten an, wie sie Tabelle 4 zeigt. Eine Gegenüberstellung der anfallenden Mengen und der maximal zulässigen Emissionsmenge ${ }^{137)}$ läßt erkennen, daß auch in Zukunft die 
pro Arbeitstag anfallenden Kr-85 Mengen kein grundsätzliches Problem bei ihrer Beseitigung darstellen werden. Man hat jedoch in Rechnung zu setzen, daß der Auflösevorgang besonders bei höheren Abbränden sehr rasch vonstatten gehen kann $(V, 2 b)$. Dann kann leicht der Fall eintreten, daß die pro Sekunde zugelassene Kr-85 Menge doch erheblich überschritten wird. Aus diesem Grunde dürfte es auf längere Sicht notwendig werden, Pufferbehälter für die dosierte Abgabe von $\mathrm{Kr}-85$ vorzusehen.

Tabelle 4. Kr-85-Bildung

\begin{tabular}{|c|c|c|c|}
\hline \multirow[b]{2}{*}{ Abbrand } & \multicolumn{2}{|l|}{$\mathrm{CiKr}-85$} & \multirow{3}{*}{$\begin{array}{l}\text { Max. zuläss. } \\
\text { Ausstoß pro } \\
\text { Tag durch } \\
80 \mathrm{~m} \text { Kamin }\end{array}$} \\
\hline & $\begin{array}{l}\text { Thermischer } \\
\text { Reaktor }\end{array}$ & $\begin{array}{l}\text { Schneller } \\
\text { Brüter }\end{array}$ & \\
\hline [MWd] & {$[\mathrm{Ci}]$} & {$[\mathrm{Ci}]$} & \\
\hline 20000 & 9000 & 2300 & \\
\hline 30000 & 13500 & 3500 & $\begin{array}{l}260000 \mathrm{Ci} / \mathrm{d} \\
\text { bzw. }\end{array}$ \\
\hline 40000 & 18000 & 4600 & $3 \mathrm{Ci} / \mathrm{sec}$ \\
\hline
\end{tabular}

Xenon-133 hat eine fünffach höhere Spaltausbeute als Krypton-85. Der maximal zulässige Xenon-133 Ausstoß in die Atmosphäre ist derselbe wie bei Krypton-85. Die Halbwertszeit des Xenon-133 beträgt jedoch nur 5,65 Tage. Eine Bedeutung im Abgasstrom würde Xenon-133 deshalb erst erhalten, wenn die Ablklingzeiten auf 30 bis 40 Tage abgekürzt würden. Eine Realisierung dieser Möglichkeit ist jedoch wegen dem damit verbundenen Jod-131-Problem nicht zu erwarten (V.2.e. $\gamma$ ).

Durch Absorption in perfluorierten Kohlenwasserstoffen ist es möglich, Edelgase selektiv aus Gasströmen zu entfernen 138). Diese Möglichkeit kann von Interesse werden, wenn ein größeres kommerzielles Interesse an radioaktiven Edelgasen entsteht.

ß) Tritium

Die Entstehung von Tritium bei der Kernspaltung ist ein gesicherter Befund 139,140,141). Pro 10000 thermischen Uranspaltungen entsteht ca. ein Atom Tritium. Bei der schnellen Spaltung von Plutonium liegen keine experimentellen Messungen vor. Theoretische Utberlegungen lassen jedoch eine Ausbeutesteigerung um den Faktor 2,5 erwarten 142,143).

Aufgrund dieser Spaltausbeuten ergeben sich als Tritiumgehalt für Brennelemente thermischer Reaktoren $130 \mathrm{Ci} \mathrm{T}$ pro $10000 \mathrm{MWd} \mathrm{Ab-}$ brand, bei schnellen Reaktoren sollen es $323 \mathrm{Ci}$ T pro $10000 \mathrm{MWd}$ sein. 
Die maximal zulässige Tritiumabgabe im Abwasser darf nur $3 \cdot 10^{-3} \mathrm{Ci} / \mathrm{m}^{3}$ betragen 142). Bei einem $80 \mathrm{~m}$ hohen Abluftkamin ist dagegen eine gasförmige Tritiumabgabe von $0,22 \mathrm{Ci}-\mathrm{T}$ pro Sekunde oder $1,9 \cdot 10^{4} \mathrm{Ci}$ pro Tag gestattet ${ }^{136)}$.

Bei einer Wiederaufarbeitungsanlage von $1 \mathrm{t}$ Brennstoff pro Tag fallen täglich rund $150 \mathrm{~m}^{3}$ an Abwasser aus der gesamten Anlage an 144) Ein Vergleich der Zahlen zeigt (Tabelle 5), daß es in der Praxis selten der Fall sein wird, die anfallenden Tritiummengen in flüssiger Form als $\mathrm{Ab}$ wasser zu beseitigen.

Tabelle 5. Tritium-Bitdung

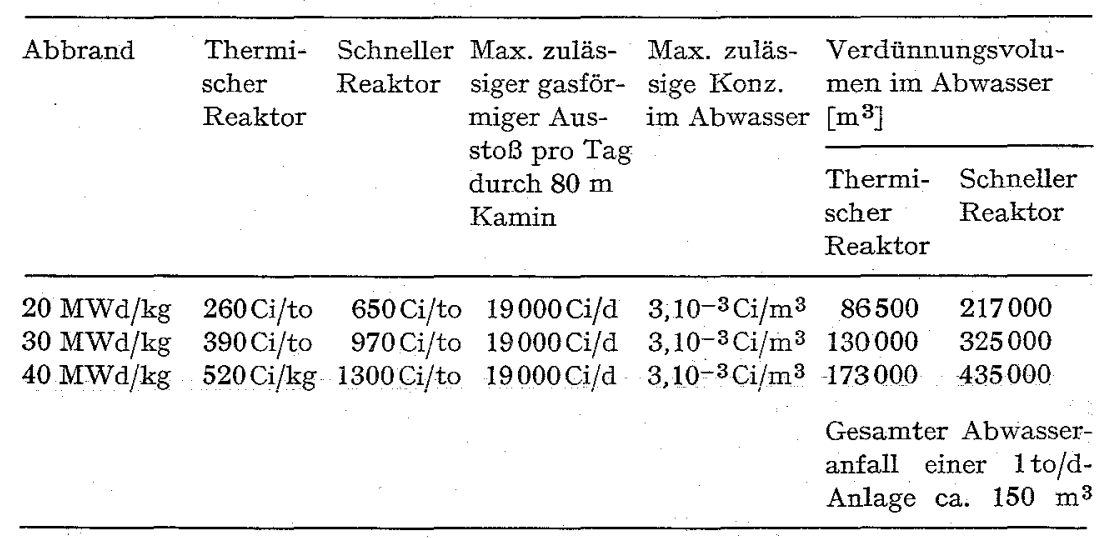

Im Prozeßablauf tritt die Hauptmenge des Tritiums in der wäßrigen Phase des ersten Extraktionscyclus zusammen mit den Spaltprodukten auf. Bei der anschließenden Konzentrierung dieser Abfallösung wird die Hauptmenge des Wassers in die Dampfphase übergeführt. An dieser Stelle ist es möglich, den Großteil des Tritiums, der auf etwa $40 \mathrm{~m}^{3}$ Wasser verteilt ist, mit der allgemeinen Abluft der Anlage zu verdünnen und über den Abluftkamin zu entfernen. Die zulässige Abluftaktivität von 19000 Ci Tritium pro Tag würde dabei keine engen Schranken setzen. Allerdings sind bei diesem Weg der Tritiumbeseitigung, die meteorologischen Verhältnisse zu berücksichtigen. Gegebenenfalls müßte durch entsprechende Verdiunnung mit Frischluft dafür gesorgt werden, daß nicht unmittelbar nach Verlassen der Kaminöffnung wieder eine Kondensation des Wasserdampfes möglich ist.

r) Jod-131 (Jod-129)

Durch die verhältnismäßig langen Halbwertszeiten von $\mathrm{Kr}-85$ (10,8 Jahre) und Tritium (12,3 Jahre) ergeben sich die anfallenden Aktivitäts- 
mengen dieser Radionuklide aus dem Abbrand. Bei Jod-131 dagegen wird durch die verhältnismäßig kurze Halbwertszeit von 8 Tagen die anfallende Aktivität von der spezifischen Stableistung bestimmt (Zahl der Spaltungen pro kg). Die Verweilzeit der Brennelemente im Reaktor wird dabei länger als 80 Tage angenommen, was in der Regel erfüllt wird.

Bei der thermischen Uranspaltung beträgt die Sättigungsaktivität von Jod-131 $250 \mathrm{Ci}$ pro $10 \mathrm{~kW}$ thermischer Reaktorleistung. Bei der schnellen Spaltung von Plutonium liegt die Jod-131 Aktivität aufgrund der höheren Spaltausbeute bei $410 \mathrm{Ci}$ Jod-131 pro $10 \mathrm{~kW}$. Daraus folgt, $\mathrm{da} ß$ bei Bestrahlungsende pro Tonne mit einer Größenordnung von $10^{5}$ Ci Jod-131 bei thermischem Brennstoff zu rechnen ist und mit 4 bis $5 \cdot 10^{6} \mathrm{Ci}$ Jod-131 bei den Zentralstäben Schneller Brüter. Diese Aktivität fällt allerdings mit einer Halbwertszeit von 8 Tagen verhältnismäßig rasch ab, Abb. 11.

Neben dem Jod-131 entsteht als weiteres Jodisotop noch Jod-129. Seine Halbwertszeit beträgt $1,6 \cdot 10^{7} \mathrm{Jahre}$, seine Aktivität $1,5 \cdot 10^{-6} \mathrm{Ci}$ pro MWd Abbrand. In einer $1 \mathrm{t} / \mathrm{d}$-Anlage werden demnach aus einem Brennstoff mit 20 bis $40 \mathrm{MWd} / \mathrm{kg}$ Abbrand pro Tag zwischen $20 \cdot 10^{-3}$ bis $60 \cdot 10^{-3} \mathrm{Ci}$ Jod-129 freigesetzt. Die Jod-131 Aktivität überwiegt deshalb auch nach 100 bis 120 Tagen Abklingzeit noch um den Faktor $2 \cdot 10^{3}$ bis $5 \cdot 10^{3}$. Die maximal zulässige Jod-129. Abgabe ist heute noch nicht festgelegt. Sie dürfte wegen der langen Halbwertszeit sicher unter dem Jod-131-Wert liegen. Es ist jedoch kaum anzunehmen, daß sie um den Faktor $10^{-3}$ unter dem Wert für Jod-131 liegt (Das Plutonium-239 liegt nur um den Faktor 0,247 tiefer). Deshalb wird auch weiterhin das Jod-131 das dominierende Problem im Abgasstrom bleiben.

Bei der Wiederaufarbeitung tritt das Jod mit über 50\% im Abgas des Auflöseprozesses auf 145,146). Durch Zugabe von Quecksilber ist es möglich, diesen Anteil auf $40-50 \%$ zu reduzieren ${ }^{145}$ ). Im anschließenden Extraktionscyclus geht das Jod zum Teil über in die organische Phase, wo es entweder einfach gelöst oder chemisch gebunden wird. Das gelöste Jod verbleibt anschließend im alkalischen Lösungsmittelwäscher. Das chemisch gebundene Jod wird durch die organische Wäsche nicht entfernt und recycliert mit dem Lösungsmittel 147,148). Wenn das Jod vorher durch Quecksilberionen in der wäßrigen Phase gehalten worden ist, so tritt es beim anschließenden Eindampfen dieser Lösung wieder im Abgas auf 149). Das in der Prozeßlösung verbliebene Jod wird teilweise ebenfalls noch in das Abluftsystem übergeführt, entweder durch die Behälterentlüftung oder beim Rühren oder pneumatischen Transport der Lösung oder der Probeentnahme.

Abgesehen von diesem chemisch schwer kontrollierbaren Verhalten des Jods innerhalb der Anlage entsteht das Jodproblem vor allem durch die relativ geringe, in der Abluft zugelassene Aktivitätsmenge. Sie be- 


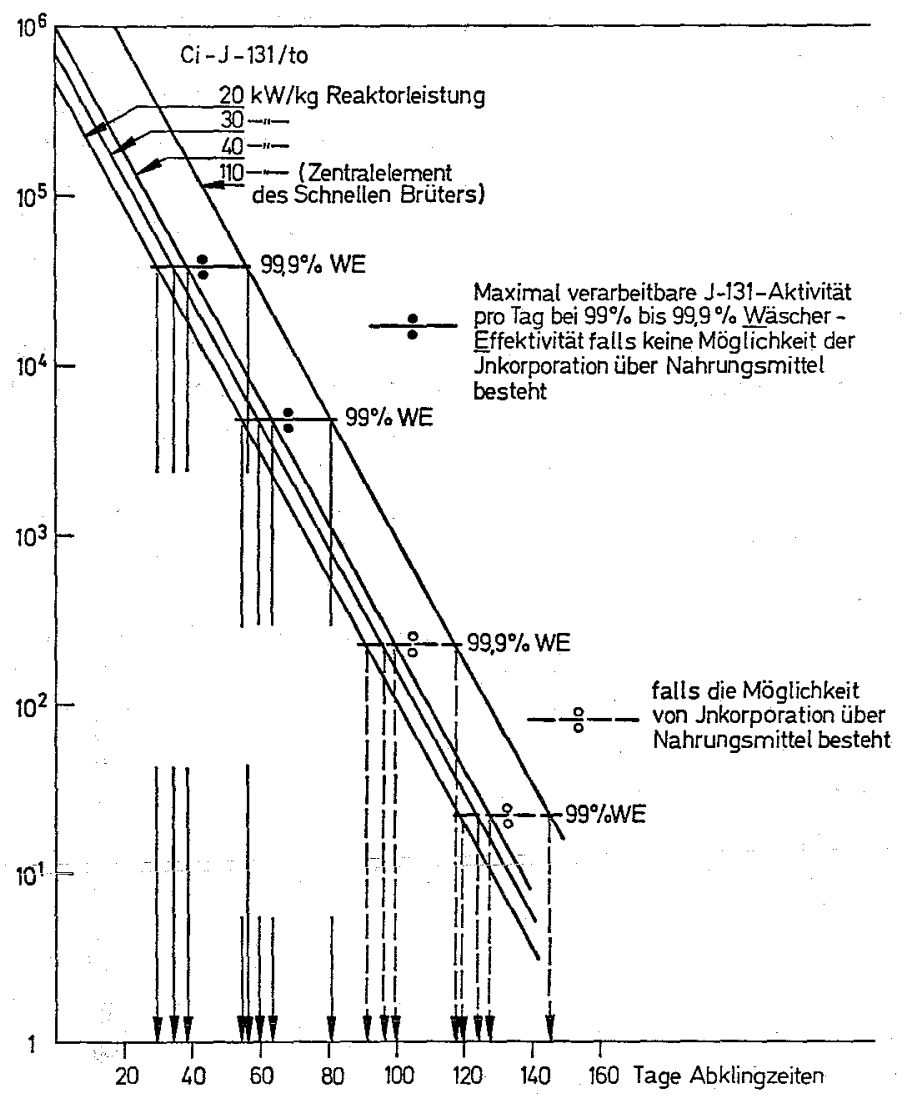

Abb. 11. Notwendige Abklingzeiten bei verschiedenen Reaktorleistungen und JodWäscher-Effektivitäten

trägt bei einer wirksamen Schornsteinhöhe von $80 \mathrm{~m}$ nur $0,22 \mathrm{Ci} / \mathrm{Tag}$ bzw. $2,5 \cdot 10^{-6} \mathrm{Ci} / \mathrm{sec}$, wenn mit einer Inkorporation durch Nahrungsmittel (Milch) gerechnet werden muß. Ohne Inkorporationsgefahr sind $48 \mathrm{Ci} /$ Tag bzw. $5,6 \cdot 10^{-4} \mathrm{Ci} / \mathrm{sec}$ zugelassen. Wie Abb. 11 zeigt, muß bei Inkorporationsgefahr das Jod-Reinigungssystem einer 1 t-Anlage bei einer Abklingzeit von 100 Tagen eine Dauereffektivität von $99,9 \%$ erreichen.

8) Die gebräuchlichen Jodabsorptionsmethoden und ihre Effektivitäten.

Das Abgas des Auflösers enthält in der Regel Stickoxide, die eine alkalische Wäsche mit $\mathrm{Na}_{2} \mathrm{CO}_{3}$ oder $\mathrm{NaOH}$ erfordern. Dadurch wird gleich- 
zeitig auch das Jod mit einer Effektivität von 95-99\% aus dem Abgasstrom entfernt 150,151). Eine zusätzliche Wäsche mit Thiosulfat erhöht diese Ausbeute nur mehr geringfügig ${ }^{152}$ ). Ebenso bringt eine zusätzliche Wäsche mit verdünnter Salpetersäure und $\mathrm{Hg}(\mathrm{I})$ und $\mathrm{Hg}(\mathrm{II})$-nitrat nur noch eine kleine Verbesserung ${ }^{153)}$.

In Kombination mit einem Aktiv-Kohlefilter jedoch erreicht die Wirksamkeit einer $\mathrm{HNO}_{3}$-Wäsche mit $0,001 \mathrm{~m} \mathrm{Hg}$ (I) und $0,001 \mathrm{Hg}$ (II)nitrat eine Effektivität von 99,96\% 154).

$\mathrm{AgNO}_{3}$-Reaktoren erreichen Wirksamkeiten von 99 bis $99,9 \%{ }^{156}$ ). Ihre Betriebstemperatur liegt bei $200^{\circ} \mathrm{C}$. Die Bildung eines $\mathrm{AgNO}_{3}$ AgJ Eutektikums mit niedrigem Schmelzpunkt kann zu Silberverlusten führen. Auch eine Passivierung durch das gebildete Silberjodid und Silberjodat 155) kann dabei eintreten. Durch Besprühen mit frischer Silbernitratlösung läßt sich das Absorberbett bis ca. 10 mal regenerieren ${ }^{157)}$.

Umfangreiche Laboruntersuchungen wurden über die Wirksamkeit von Aktivkohlefilter durchgeführt, undWirksamkeiten von 90 bis $99,999 \%$ werden berichtet. Dabei sind die Art der Tierkohle, die Durchsatzgeschwindigkeit, die Temperatur, die Feuchtigkeit, das Trägergas, die Verunreinigungen des Trägergases und auch die physikalische und chemische Form der Tierkohle von Bedeutung 149,158, 159,160, 161). Die Berichte aus technischen Anlagen sprechen dagegen nur von Trenneffektivitäten zwischen 88 und $99 \% 153,162,163,164)$; der Grund hierfür düıfte in der allmählich schwindenden Absorptionskapazität der Aktivkohle liegen ${ }^{162}$ ). Nachteilig bei der Anwendung von Aktivkohlefiltern ist außerdem die Brandgefahr beim Kontakt mit oxidierenden Gasen 165,166,167).

Die in technischen Anlagen zu beobachtende Abnahme der Wirksamkeit von Aktivkohlefiltern wird z.T. auch auf die Gegenwart des Jods in Form von Alkyljodiden zurückgeführt. Die Möglichkeit der Bildung von aliphatischen Jodverbindungen besteht im Kontakt des Radiojods mit dem organischen Extraktionsmittel. Zur Entfernung der Alkyljodide aus dem Abgasstrom wurden bisher zwei Methoden angewendet: eine Isotopenaustauschreaktion und eine chemische Reaktion mit Alkylaminen.

Die Isotopenaustauschreaktion spielt sich auf einer mit inaktivem Jod imprägnierten Tierkohle ab 168,169,170). Die erreichte Effektivität betrug $99,99 \%$ bei einer relativen Feuchtigkeit unter $0,1 \%$. Bei $90 \%$ relativer Feuchtigkeit belief sich die Trenneffektivität noch auf 99,5 bis $99,99 \%$.

Die andere Methode beruht aus einer chemischen Reaktion des Alkyljodids mit Alkylaminen, die auf Tierkohle absorbiert sind. Die erreichten Trenneffektivitäten liegen hier bei 99,9 bis $99,98 \% 171$ ).

Nach dem heutigen Stand der Technik können im Dauerbetrieb Dekontaminationsfaktoren von Jod in der Größenordnung von $99 \%$ bis $99,9 \%$ erreicht werden. Das wird für die Wiederaufarbeitung von ther- 
mischen Reaktoren auch bei zukünftigen spezifischen Stableistungen von $40 \mathrm{~kW} / \mathrm{kg}$ ausreichend sein, falls eine Abklingzeit von 100 bis 130 Tagen in Kauf genommen wird (Abb. 11). Der Mehrgehalt an Jod-131 bei den Zentralelementen des Schnellen Brüters ist durch die Verlängerung der Abklingzeit um 2 bis 3 Wochen zu kompensieren.

Die heute bekannten Methoden der Jodabsorption würden jedoch auch für thermische Reaktoren nicht mehr ausreichen, wenn die Abklingzeit wesentlich unter hundert Tage gesenkt werden soll und gleichzeitig der Inkorporationswert von Jod-131 im Abgas erreicht werden müßte.

\section{Gewinnung von Batterie-Nucliden}

\section{Die Batterie-Nuclide und ihre Bildung in Reaktoren}

Unter Batterie-Nucliden sind solche Radioisotope $\mathrm{zu}$ verstehen, die durch Selbstabsorption der radioaktiven Zerfallsenergie eine so starke Wärmeentwicklung erfahren, daß sie Energiequellen darstellen. Tabelle 6 zeigt eine Auswahl der in Frage kommenden Nuclide. Wir wollen uns im folgenden auf die Aktinoide unter den Batterienucliden beschränken.

\begin{tabular}{l} 
Tabelle 6. In Kernbrennstoffen vor- \\
kommende Batterienuclide und ihre \\
Leistungsdichten \\
\hline $\mathrm{Pu}-238$ \\
$\mathrm{Am}-241$
\end{tabular}

Die besonderen Vorteile der nuclearen Batterien sind die relativ stabile und langfristig wartungsfreie Energieabgabe (Abb. 12) bei hoher Leistungsdichte 172). Anwendung 173,174,175) finden einzelne Batterienuclide schon in der Raumfahrt und Ozeanografie, wo sie zur Energieversorgung besonders der Meß- und Nachrichtensendeeinrichtungen der Satelliten und M̃eß̉bojen dienen. Potentielle Anwendungen sind die unabhängige Stromversorgung von Relaisstationen, der kontinuierliche Antrieb mechanischer Systeme (z.B. Uhren), Mini-Batterien in der Medizin für Herzschrittmacher und Blutpumpen, Wärmequellen bei UnterwasserExpeditionen usw. 
F. Baumgärtner und H. Philipp

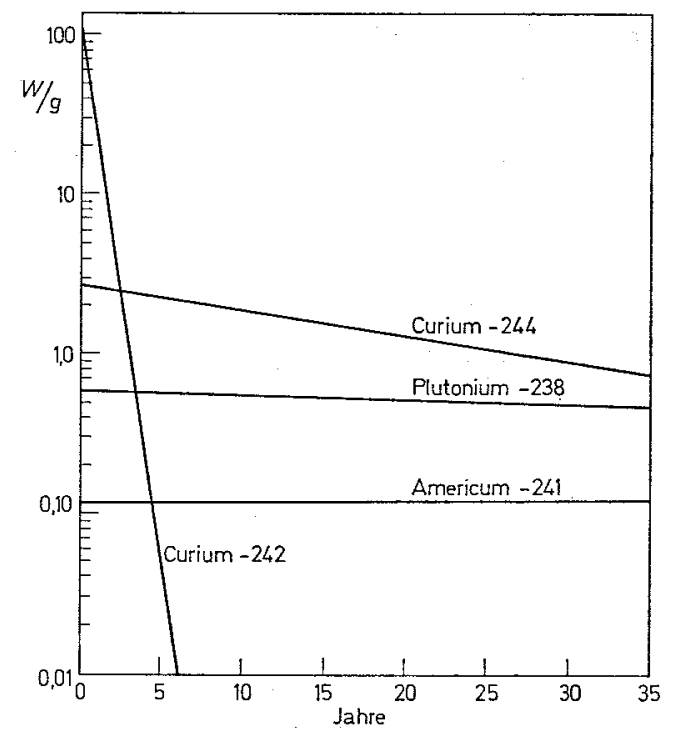

Abb. 12. Zeitlicher Verlauf der Leistungsdichten der Transurane Pu-238, Am-241, $\mathrm{Cm}-242, \mathrm{Cm}-244$

Obwohl in verbrauchten Kernbrennstoffen die meisten der Batterienuclide direkt oder in Form ihrer Vorprodukte in beachtlichen Mengen anfallen 176) (Tabelle 7) ist ihre routinemäßige Gewinnung heute noch sehr wenig entwickelt und der Preis dieser Nuclide entsprechend hoch (Bezugspreis bei der USAEC z.Z. $1 \mathrm{~g} \mathrm{~Np}-237=1000 \$, 1 \mathrm{mg} \mathrm{Cm}-244$ oder Am-243 = $1000 \$$ ).

Das Plutonium-238 ist nicht unmittelbar aus verbrauchtem Reaktorbrennstoff zu gewinnen, weil es dort gemeinsam mit den anderen Plutonium-Isotopen vorliegt und in dem Isotopengemisch weniger als $1 \%$ aus-

Tabelle 7. Gramm Nuclid pro Tonne Brennstoff 176,178)

\begin{tabular}{|c|c|c|}
\hline & $\begin{array}{l}\text { im } \\
\text { Druckwasser Reaktor } \\
\text { Yankee } \\
\text { bei } 22,4 \mathrm{MWd} / \mathrm{kg} \\
691 \mathrm{~d} \text { Ábklingzeit }\end{array}$ & $\begin{array}{l}\text { im } \\
\text { Siedewasser Reaktor } \\
\text { Vallecitos } \\
\text { bei } 16 \mathrm{MWd} / \mathrm{kg} \\
476 \mathrm{~d} \text { Abklingzeit }\end{array}$ \\
\hline $237 \mathrm{~Np}$ & 455 & 140 \\
\hline${ }^{241} \mathrm{Am}$ & 119 & 45 \\
\hline $242 \mathrm{Cm}$ & 1,0 & 0,39 \\
\hline $244 \mathrm{Cm}$ & 7,9 & 0,48 \\
\hline
\end{tabular}


macht. Seine Herstellung geht deshalb im ersten Schritt über die Isolierung von Neptunium-237, das anschließend durch separate Bestrahlung in Neptunium-238 übergeführt wird und sich durch $\beta$-Zerfall mit 2,3 Tagen Halbwertszeit spontan in Plutonium-238 umwandelt.

Das Neptunium-237 entsteht in Kernreaktoren auf zwei Wegen. Es kann einerseits aus Uran-238 mit schnellen Neutronen durch $(n, 2 n)$ Reaktion gebildet werden:

$$
238 \mathrm{U}+\mathrm{n}_{\text {schnell }} \rightarrow 2 \mathrm{n}+{ }^{237} \mathrm{U} \stackrel{2,1 \mathrm{~d}, \beta^{-}}{\longrightarrow} 237 \mathrm{~Np}\left(2,2 \cdot 10^{6} \mathrm{a}, \alpha\right)
$$

Andererseits bleibt beim Neutroneneinfang im Uran-235 in 15\% der Fälle der Uran-236 Kern erhalten. Durch einen weiteren Neutroneneinfang entsteht daraus Uran-237 mit spontanem $\beta$-Zerfall zu Neptunium237.

Der Wirkungsquerschnitt der (n, 2n)-Reaktion für Reaktor-Neutronen ist jedoch verhältnismäßig gering $\left(6 \cdot 10^{-27} \mathrm{~cm}^{2}\right)$. Deshalb fällt der Neptunium-Anteil, der aus Uran-238 gebildet wird, bei Reaktoren mit mehr als 1\% Uran-235 nicht mehr sehr ins Gewicht 177). Bei den konventionellen Druck- und Siedewasser-Reaktoren, deren Uran-235-Gehalt einige Prozent beträgt, stammt die Hauptmenge des Neptuniums deshalb vom Uran-235. Die Ursache dafür liegt aber nicht allein in der Erhöhung der Uran-Ausgangsmenge an sich. Einerseits wird durch den erhöhten Uran235-Gehalt und die damit verbundene starke Absorption thermischer Neutronen die mittlere Neutronenenergie im Reaktor gesteigert. Andererseits steigt beim Uran-236 der Wirkungsquerschnitt für Neutroneneinfang gegenüber Kernspaltung mit der Neutronenenergie erheblich an 178), so daß mit der „Härtung" des Neutronenspektrums gleichzeitig auch die Bildungswahrscheinlichkeit des Neptuniums ansteigt.

Druckwasserreaktoren haben in der Regel einen kleineren Reaktorkern als vergleichbare Siedewasser-Reaktoren. Bei gleichem Spalt-Potential enthalten Druckwasser-Reaktoren deshalb eine höhere Uran-235 Konzentration (Anreicherung). Folge davon ist wiederum ein , härteres" Neutronenspektrum in Druckwasser- als in Siedewasser-Reaktoren und eine entsprechend größere Neptunium-Bildung in diesem Reaktortyp, Tabelle 7 .

Dieselbe Erklärung trifft auch für den höheren Gehalt an Americium241 und Curium-242/244 in Druckwasser-Reaktoren zu, Tabelle 7. Mit Zunahme des epithermischen Neutronenflusses steigt auch bei den Plutonium-Isotopen 239, -240 und -241 sowie beim Americium-241 und-234 die Wahrscheinlichkeit des Kernaufbaus gegenüber der Kernspaltung erheblich an und erhöht damit die Ausbeuten an Batterie-Nucliden 178). 


\section{Die Gewinnung von Neptunium-237}

Unter den Purex-Bedingungen werden die Oxidationsstufen(VI) und (IV) des Neptuniums verhältnismäßig gut und das Neptunium(V) praktisch nicht extrahiert. Dadurch läßt sich der Weg, den das Neptunium im Purex-Prozeß nimmt, je nachdem, in den Produkt- oder Waste-Strom steuern.

Unmittelbar nach dem Auflösevorgang liegt das Neptunium, bedingt durch die entwickelten Stickoxide, in der 5-wertigen Stufe vor 179). Durch anschließendes Erhitzen der Lösung lassen sich das Nitrit und die Stickoxide beseitigen und die Oxidation des Neptunium(V) zu (VI) mit Salpetersäure vollziehen. Geringe Mengen an Nitrit wirken dabei beschleunigend.

In den Hanford-Werken wird das Neptunium zusammen mit Uran und Plutonium extrahiert ${ }^{180}$ ). Bei der anschließenden Uran-PlutoniumTrennung mit Eisen-(II) wird Neptunium(VI) dann zu Neptunium(IV) reduziert und bleibt deshalb weiterhin beim Uran, von dem es nach Oxidation zu Neptunium(V) im 2. Uran-Cyclus abgetrennt wird.

In den Savannah-River-Anlagen wird umgekehrt verfahren 181). Durch eine zusätzliche Zugabe von Nitrit wird das Neptunium(V) nach der Auflösung in der salpetersauren Lösung stabilisiert und damit in den HAW geleitet. Von dort aus erfolgt in einem erneuten Trennverfahren die Isolierung des Neptuniums über den Hexanitrato-Komplex der vierwertigen Oxidationsstufe mittels Anionenaustauscher.

\section{Americium und Curium aus dem hochaktiven Abfall}

Americium und Curium fallen als Selten-Erd-ähnliche Elemente bei der Wiederaufarbeitung unvermeidlich zusammen mit den radioaktiven Spaltprodukten im HAW (vgl. Abb. 7) an. Im Gegensatz zu Neptunium wurden sie bisher daraus aber nur in seltenen Fällen isoliert 188). Bisher wurden $\mathrm{zu}$ ihrer Gewinnung vielmehr eigene Bestrahlungsprogramme durchgeführt 182,183,184). Die dazu ausgearbeiteten Verfahren können aber als richtungsweisend betrachtet werden für die Entwicklung weiterer Prozesse zur Gewinnung von Americium und Curium aus Abfall-Lösungen. Mit einer solchen Entwicklung ist durch den wachsenden Anfall hochabgebrannter Kernbrennstoffe mit großer Wahrscheinlichkeit zu rechnen.

In dem allerdings unwahrscheinlichen Fall, daß das Kraftwerksplutonium nicht mehr als Kernbrennstoff verwendet werden sollte, ließe sich speziell das Americium-241 in größeren Mengen auch aus diesen Lagerbeständen gewinnen 178,185,186,187).

Die Isolierung von Americium und Curium entweder aus Spaltproduktlösungen der Wiederaufarbeitung ${ }^{188)}$ oder aus bestrahlten Pluto- 
nium 182,183,184) oder Americium 189,190)-Proben wurde bisher in drei Schritten durchgeführt: zuerst eine Abtrennung beider Elemente gemeinsam mit den Seltenen Erd-Spaltprodukten von allen übrigen Elementen, dann die Trennung von den Seltenen Erden und schließlich die gegenseitige Trennung der Transurane.

Für den ersten gemeinsamen Trennschritt wurden in den einzelnen Fällen Extraktionen mit TBP 191,192), mit aliphatischen Aminen 182) oder quarternären Ammoniumnitraten 193,194,195) benützt. Alle diese Verfahren erfordern jedoch eine hohe Aussalzkonzentration. Vorteilhafter für technische Anwendungen dürfte deshalb eine saure phosphororganische Verbindung wie beispielsweise Di-(2 äthylhexyl)-phosphat (HDEHP) sein, das kein Aussalzmittel benötigt 196,197,198).

Für die nachfolgende Ȧktinoiden-Lanthanoiden Trennung gibt es mehrere Vorschläge. Unter der Bezeichnung "Tramex-Prozeß" wurde ein Extraktionsverfahren aus konzentrierter LiCl-Lösung mit tertiären Aminen bekannt 182,199,200,201). Weiterhin wurde eine selektive Extraktion der Transurane in Form quartärer Ammoniumthiocyanate 202,203) aus konzentrierter Ammonrhodanidlösung vorgeschlagen. In entsprechender Weise gibt es auch Anionenaustauschprozesse aus konzentrierter Chlorid- 190,204,205) oder Thiocyanat-Lösung 185,206,207) und Verfahren mit Kationenaustauschern 208).

Die Erfahrung aus der Entwickiungsgeschichte der Wiederaufarbeitung lehrt jedoch, daß sich in der Technik letzten Endes diejenigen kontinuierlich arbeitenden (Extraktions-) Prozesse durchsetzen, deren Prozeßchemikalien entsprechend strahlenbeständig sind, die nur geringe Abfallvolumina liefern (d.h. keine Aussalzmittel notwendig haben) und deren Abfallösung bei der anschließenden langzeitigen Lagerung kein schwerwiegendes Korrosionsproblem aufwirft (was bei Chloridlösungen beispielsweise der Fall wäre). Unter diesem Gesichtspunkt sind zwei Verfahren besonders erwähnenswert. Der sogenannte ,Talspeak-Prozeß“ 201 , 209,210) extrahiert mit einer sauren Organophosphorverbindung (HDEHP) selektiv die Lanthanoide und läßt Americium und Curium mit den Komplexbildnern DTPE (Diäthylentriamin-penta-Essigsäure) und Milchsäure in der wäßrigen Phase zurück.

Der andere Prozeß 211) extrahiert zuerst mit HDEHP das Americium und Curium zusammen mit den Lanthanoiden und anderen Verunreinigungen wie Zirkon, Niob, Eisen etc. aus einer salpetersauren, Citronenoder Weinsäure-haltigen Lösung im $\mathrm{p}_{\mathrm{H}}$-Bereich von 1-3. Anschließend foigt die Rückextraktion der reinen Americium-Curium-Fraktion durch selektive Komplexbildung mit einer Milchsäure-DTPA-Lösung 209,210). Das Extraktionsmittel wird mit Salpetersäure wieder regeneriert.

Im dritten Schritt, bei der Trennung von Americium und Curium wurde bisher eine Elutionschromatographie von einem Anionenaustau- 
scherbett mit $4 \mathrm{n} \mathrm{LiNO}_{3}$ 194,212,213,214) oder einem Methanol-Salpetersäure-Gemisch 215) eingesetzt. Es gibt aber auch die Möglichkeit 216) der selektiven Oxidation und Fällung des Americiums als $\mathrm{K}_{3} \mathrm{AmO}_{2}\left(\mathrm{CO}_{3}\right)_{2}$ aus einer $\mathrm{K}_{2} \mathrm{CO}_{3}$-Lösung. Die Entwicklung auf diesem Gebiet ist noch sehr im Fluß. Für einen kontinuierlichen, technischen Betrieb steht eine befriedigende Lösung noch aus.

\section{Literatur}

1) Jelinek-Fink, P., u. B. Skorpil: Angereichertes $\mathrm{UF}_{6}$ - Preise bei Bezug von der USAEC. Atomwirtschaft 13, 365 (1968).

2) Rometsch, R.: Wiederaufarbeitung abgebrannter Kernbrennstoffe in Europa Aufgaben der Eurochemic. Atomwirtschaft 11, 423 (1966).

3) Zusammengestellt nach Angaben von $H$. Grümm et al. Ergänzendes Material zum Bericht: Kernbrennstoffbedarf und Kosten verschiedener Reaktortypen in Deutschland, KFK-466 (1966).

4) Kennedy, J.W., G. T. Seaborg, E. Segrè, and A.C. Wahl: Fissionable Isotope of a New Element: $94^{239}$. Phys. Rev. 70, 555 (1946).

5) Symposium on Chemical Processing of Irradiated Fuels, Hanford 1959. USReport TID-7583 (1959).

6) Stoller, S. $M$., and R. B. Richards: Reactor Handbook, Vol. II, S. 124. Fuel Processing (1961).

7) Seaborg, G, T.: The Plutonium Story in the Transuranium Elements. Edison Wesley 1958.

8) Thompson, S. G., and G. T. Seaborg: The First Use of Bismuth Phosphate for Separating Plutonium from Uranium and Fission Products, Process Chemistry. Londion: Pergamon Press 1956.

9) Lawrowski, S., L. R. Dawson, and I. E. Tepe: Solvent Extraction Process for Concentration and Isolation of Product. US-Report CN-2511 (1945).

10) Perlman, J.: Separation Prucesses. US-Report CN-3627 (1946).

11) Culler Jr., F. L., and $F$. R. Bruce: The Processing of Uranium-Aluminium Fuel Elements. Proc. Intern. Conf. Peaceful Uses At. Energy, Geneva 9, 484 (1955).

12) Flagg, J. F.: Chemical Processing of Reactor Fuels. New York: Academic Press 1961.

13) Lawrowski, S., and $M$. Levenson: Redox Process - A Solvent Extraction Processing Method for Irradiated Uranium, Process Chemistry, Vol. II. USReport TID-7534 (1957).

14) Warf, J. C.: Extraction of Ce(IV) Nitrate by Butylphosphate. J. Am. Chem. Soc. 71, 3257 (1949); ursprünglich US-Reports CC-2402 (1945) und ISC-8 (1947).

15) Ellison, C. V., D. E. Ferguson, and T.C. Runion: Solvent Extraction Recovery from Metal Waste. US-Report ORNL-258 (1949).

16) Ferguson, D. E., and T.C. Runion: Tributylphosphate Solvent Extraction of U from Metal Waste. US-Report ORNL-260 (1949).

17) Culler, F. L.: Reprocessing of Reactor Fuel and Blanket Materials by Solvent Extraction. Proc. Intern. Conf. Peaceful Uses At. Energy, Geneva 9, 467 (1955).

18) Gresky, A. T.: Solvent Extraction Separation of U-233 and Thorium from Fission Products by Means of Tributyl-Phosphate. Proc. Intern. Conf. Peaceful Uses At. Energy, Geneva 9, 505 (1955). 
19) Lanham, W. B., and T. C. Runion: Purex Process for Pu and U Recovery. US-Report ORNL-479 (1949).

20) Goldschmidt, B., P. Regnaut, and $J$. Prevot: Solvent Extraction of Plutonium from Uranium Irradiated in Atomic Piles. Proc. Intern. Conf. Peaceful Uses At. Energy, Geneva 9, 492 (1955).

21) Flanary, I. R.: Solvent Extraction Separation of Uranium and Plutonium from Fission Products by Means of Tributyl-Phosphate. Proc. Intern. Conf. Peaceful Uses At. Energy, Geneva 9, 528 (1955).

22) Irish, E. R., and W. H. Reas: The Purex Process. US-Report HW-49483A.

23) Campbell, W. M.: Canadian Fuel Processing. Nucleonics 14, 92 (1956).

24) Gowing, M.: Britain and Atomic Energy 1939-1945. London: Macmillan 1964.

25) Howells, G. R., T. G. Hughes, D. R. Mackey, and K. Saddington: The Chemical Processing of Irradiated Fuels from Thermal Reactors. Proc. 2. Intern. Conf. Peaceful Uses At. Energy, Geneva 17, 3 (1958).

26) Fletcher, $J$. $M$.: Ethers as Extractants. In: Aqueous Processing Chemistry for Irradiated Fuels, Brüssel 1963, ENEA-Eurochemic.

27) Warner, B. F.,W.W. Marshall, A. Naylor, and G. D. C. Short: The Development of the New Separation Plant, Windscale. Proc. 3. Intern. Conf. Peaceful Uses At. Energy, Geneva 10, 224 (1964).

28) Baroncelli, F., G. Galleri, A. Moccia, G. Scibona, and M. Zifferero: The Eurex Flowsheet: Processing of Irradiated U-Al Alloys by Amine Solvent Extraction. Comitato Nazionale Energia Nucleare, Rom 1963.

29) Zifferero, M.: Proposed Flowsheets for Amine Systems, Aqueous Processing Chemistry for Irradiated Fuels, Brüssel 1963, ENEA-Eurochemic.

30) Brown, K. B., C.F. Coleman, D.J. Crouse, J.O. Denis, and J.G. Moore: The - Use of Amines Extractants for Uranium from Acidic Sulfate Liquors. US-Report AECD-4142 (1954).

31) Moore, J.G., K. B. Brown, and C. F. Coleman: Further Studies of Amines as Extractants for Uranium from Acid Sulfate Solutions. US-Report AECD-4145 (1955).

32) Arnold, $W, D$., and $D . J$. Crouse: Further Evaluation of Amines as Extractants for Uranium from Sulfate Liquors. US-Report ORNL-3030 (1961).

33) Coleman, C. F., K. B. Brown, J. G. Moore, and D. J. Crouse: Solvent Extraction with Alkyl Amines. Ind. Eng. Chem. 50, 1756 (1958).

34) Brown, K. B., C. F. Coleman, D. J. Crouse, C. A. Blake, and A.D.Ryon: Solvent Extraction Processing of Uranium and Thorium Ores. Proc. 2. Intern. Conf. Peaceful Uses At. Energy, Geneva 3, 472 (1958).

35) Diamond, R. M., and D. G. Tuck: Extraction of Inorganic Compounds into Organic Solvents. Progr. Inorg. Chem. 2, 157 (1960).

36) Naylor, A.: Fission Product Chemistry in Relation to TBP Processes, in Reprocessing of Fuel from Present and Future Power Reactors. Kjeller-Report KR-126, S. 101 (1967).

37) Brown, P.G.M., J. M. Fletcher, C.J.Hardy, J.Kennedy, D. Scargill, A. G. Wain, and $J$. L. Woodhea $a$ : The Significance of Certain Complexes of Ruthenium, Niobium, Zirconium, and Uranium in Plant Processes. Proc. 2. Intern. Conf. Peaceful Uses At. Energy, Geneva 77, 118 (1958).

38) Scargill, D., C. E. Lyon, N.R. Large, and $J . M$. Fletcher: Nitratoaquo Complexes of Nitrosylruthenium-III, J. Inorg. Nucl. Chem. 27, 161 (1965).

39) Klaas, $J .:$ RuNO-Nitro Complexes. Conversion Rates and Extraction Coefficients, Proceedings XI. Intern. Conf. on Coordination Chemistry 1968. Amsterdam: Elsevier Publ. Co. 1968. 
40) Wallace, R. M.: The Composition of Some Nitrato Nitrosylruthenium Complexes. J. Inorg. Nucl. Chem. 20, 283 (1961).

41) Fletcher, J. M., C. E. Lyon, and A. G. Wain: Partion Coefficients of Nitratonitrosylruthenium Complexes between Nitric Acid and TBP-Phases. J. Inorg. Nucl. Chem. 27, 1841 (1965).

42) Broren, P. G. M.: Nitrato Complexes of Nitrosylruthenium. J. Inorg. Nucl. Chem. 13, 73 (1960).

43) Finsterwalder, L.: Über die Extraktionskinetik von PIutonium IV und Uran VI im Purex-Prozeß. Dissertation TH München 1968.

44) Ortega, J., L. R. Salvador, and B. Lopez-Perez: The Use of Tartrates for the Separation of Uranium(VI) and Plutonium(IV) by Extraction with TBP, Solvent Extraction Chemistry, S. 335. New York: John Wiley 1967.

45) Naylor, A.: Plutonium-Uranium Separation Techniques in TBP Systems, in Reprocessing of Fuel from Present and Future Power Reactors. Norwegischer Report KR-126, S. 172 (1967).

46) Edwall, B.: Some Experience in the Use of Uranium(IV) Nitrate, in Aqueous Reprocessing Chemistry for Irradiated Fuels, Brüssel 1963, ENEA-Eurochemic.

47) Lopez-Menchero, E., L. Gehem, H. Eschrich, P. Hansen, J. Centeno, and R. Aerts: Study of Uranium(IV) Nitrate as Reductant for Plutonium. I. The Preparation of Uranium(IV) Nitrate Solutions. Eurochemic-Report ETR-180.

48) Biddle, P., H. A. C. McKay, and J.H. Miles: The Role of Nitrous Acid in the Reduction of PIutonium(IV) and Uranium(VI) by Uranium(IV) in TBP Systems. In: Solvent Extraction Chemistry of Metals, S. 133. London: Macmillan 1965.

49) McKay, H. A. C.: Uranium(IV) Nitrate as a Reducing Agent for UraniumPlutonium Separation, in Aqueous Reprocessing Ghemistry for Irradiated Fuels, S. 281. Brüssel 1963, ENEA-Eurochemic.

50) Talmont, L. R. : Utilization of Uranium(IV) Nitrate in a Second Cycle of Purification of Plutonium by TBP. In: Solvent Extraction Chemistry of Metals, S. 103. London: Macmillan 1965.

51) Regnault, P., D. Faugeras, A. Brut, R. Helon, and A. Redon: The Processing of Irradiated Uranium in the Fontenay aux Roses Pilot Plant. Proc. 2. Intern. Conf. Peaceful Uses At. Energy, Geneva 17, 73 (1958).

52) Karraker, D. G.: Temperature Effects on TBP Solvent Extraction Processes. Proc. 2. Intern. Conf. Peaceful Uses At. Energy, Geneva 17, 333 (1958).

53) Eurochemic First Activity Report 1959-1961. OECD-ENEA Paris 1961.

54) Stoller, S. M., and R. B. Richards: Reactor Handbook, Vol. II, S. 154/155. Fuel Processing (1961).

55) Hoffart, $A . J$, and $R . D$. Thomson: Effects of $\mathrm{ZrO}\left(\mathrm{NO}_{3}\right)_{2}$ and $\mathrm{HNO}_{3}$ upon the Chemical Stability of TBP. US-Report IDO-14643 (1967).

56) Hardy, C. J., and D. Scargill: Extraction of Zr from Nitrate Solution by DBP. J. Inorg. Nucl. Chem. 17, 337 (1961).

57) Fangeras, $P$., and $X$. Talmont: Radiolysis and Hydrolysis of TBP and their Effects, Proc. XI. Intern. Conf. Coordination Chemistry 1968. Amsterdam: Elsevier Publ. Co. 1968.

58) Blake Jr., C. A.: Solvent Stability in Nuclear Fuel Processing, Evaluation of the Literature, Calculation of Radiation Dose and Effects of Iodine and Plutonium. US-Report ORNL-4212.

59) McKay, H. A. C.: TBP-Meeting-Point of Science and Technology, Solvent Extraction Chemistry, S. 185. Amsterdam: North-Holland Publ. Co. 1967.

60) Vogel, R. C., A. A. Jonke, and R. K. Steunenberg: The Non Aqueous Processing of Spent Fast Reactor Fuels, Symposium on Dry Reprocessing, Mol. 1968. 
61) Jonke, A. A.: Reprocessing of Nuclear Reactor Fuels by Processes Based on Volatilization, Fractional Distillation, and Selective Adsorption. At. Energy Rev. 3, (1), 3-60 (1965).

62) Vogel, R. C., W. H. Carr, G. I. Cathers, J. Fischer, L. P. Hatch, R. W. Horton, A. A. Jonke, R. P. Milford, J.J. Reilly, and G. Strickland: Fluoride Volatility Processes for Recovery of Fissionable Materials from Irradiated Reactor Fuels. Proc. 3. Intern. Conf. Peaceful Uses At. Energy, Geneva 10, $491-498$ (1964).

63) Schmets, J., G. Camozzo, A. Francesconi, P. Godrie, R. Heremans, G. Pierini, and $P$. Speeckaert: Reprocessing of Nuclear Fuels by Volatilization. Proc. 3. Intern. Conf. Peaceful Uses At. Energy, Geneva 10, 520-529 (1964).

64) Bourgeois, $M$, and $P$. Faugeras: The Processing of Irradiated Fuels by the Halogens and their Compounds. Proc. 3. Intern. Conf. Peaceful Uses At. Energy, Geneva 10, 483-490 (1964).

65) Pievce, R. D., and L. Burris Jr.: Pyroprocessing of Reactor Fuels. In: Reactor Technology, Selected Reviews. US-Report TID-8540, S. 411-476 (July 1964).

66) Lawroski, S., and L. Burris Jr.: Processing of Reactor Fuel Materials by Pyrometallurgical Methods, At. Energy Rev. 2(3), 3-69 (1964).

67) Faugeras, P.: Chemical Treatment of Irradiated Nuclear Fuels. Energie Nucl. 7, 214-227 (1965).

68) Burris, L., K. M. Harmon, G. E. Brand, E. W. Murback, and R. K. Steunenberg: Pyrometallurgical and Pyrochemical Fuel Processing. Proc. 3. Intern. Conf. Peaceful Uses At. Energy, Geneva 10, 501-510 (1964).

69) Benedict, G. E., W. R. Bond, G. Jansen Jr., L. G. Morgan, and J. R. Lundquist: Status of the Salt Cycle Process for Processing Oxide Fuels. US-Report BNWLSA-205 (Sept. 1965).

70) Burris, L., and G. A. Bernett: Dry Processes. Reactor Fuei Process. $9(1), 36-38$ (1965-1966).

71) Reas, $W . H .:$ The Aqua-Fluor Process. In: Reprocessing of Fuel from Present and Future Power Reactors. Kjeller-Report KR-126, S. 361.

72) - Commercial Nuclear Fuel Recovery. In: Reprocessing of Fuel from Present and Future Power Reactors. Kjeller-Report KR-126, S. 342.

73) Uriarte, $A . L$., and $R$. H. Rainey: Dissolution of High Density $\mathrm{UO}_{2}, \mathrm{PuO}_{2}$ and $\mathrm{UO}_{2}-\mathrm{PuO}_{2}$ Pellets in Inorganic Acids. US-Report ORNL-3695 (April 1965).

74) $B \ddot{a} h r, W$. , u. T. Dippel: Über die Auflösung von $\mathrm{PuO}_{2}$-haltigen Brüterbrennstoffen in Salpetersäure für die wäßrige Wiederaufarbeitung nach dem PurexVerfahren. KFK-673 (1967).

75) Goode, J. H.: Hot Cell Dissolution of Highly Irradiated $20 \% \mathrm{PuO}_{2}-80 \mathrm{UO}_{2}$ Fast Reactor Specimens. US-Report ORNL-3754 (October 1965).

76) Baumgärtel, G., W. Ochsenfeld u. H. Schmieder: Die Verteilung der Metallnitrate im System $\mathrm{Pu}\left(\mathrm{NO}_{3}\right)_{4}-\mathrm{UO}_{2}\left(\mathrm{NO}_{3}\right)_{2}-\mathrm{HNO}_{3} / \mathrm{TBP}-\mathrm{Dodecan}$, KFK-680 (1967).

77) Ochsenfeld, W., H. Schmieder u. S. Theiß: Wäßrige Wiederaufarbeitung der Brennelemente Schneller Brüter. I. Die gemeinsame Extraktion und Trennung von Makromengen Plutonium und Uran im Purex-Prozeß. KFK-911 (1969).

78) Baumgärtner, $\bar{F} ., \bar{W}$. Ochsenfeld, $B$. Roth u. L. Finstervalder: Das Verhalten hoher Plutoniumkonzentrationen im Purex-Prozeß und die Entwicklung schnelier Extraktoren zur Wiederaufarbeitung von Kernbrennstoffen. KFK652 (1967).

79) Rainey, R. H.: Hydrogen Reduction of $\mathrm{Pu}(\mathrm{IV})$ to $\mathrm{Pu}(\mathrm{III})$. Nuci. Appl. 1, 310 (1965).

81) Stoller, S. M., and R. B. Richards: Reactor Handbook, Vol. II, S. 445. Fuel Processing-(1961): 
82) Jouannaud, C.: Discussion Fuel Reprocessing (I) Expérience de six années de fonctionnement de l'usine de retraitement de Marcoule. Proc. 3. Intern. Peaceful Uses At. Energy, Geneva 10, 215 (1965).

83) Nicholson, E. L.: Preliminary Investigation of Processing Fast-Reactor Fuel in an Existing Plant. US-Report ORNL-TM-1784 (May 1967).

84) Nuclear Fuel Services, Inc., Safety Analysis - Spent Fuel Reprocessing Plant, Vol. II (July 1962).

85) Lewis, W.H., M.E. Weech, and B.E. Knight: Criticality Control in the Nuclear Fuels Services Processing Plant. US-Report SC-DC-67-1305. Nuclear Criticality Safety Conference (ANS), Las Vegas, Nevada (December 1966).

86) U.S. Atomic Energy Commission, Division of Operational Safety, Operational Accidents and Radiation Exposure Experience within the USAEC, 1943-1964. U.S. Government Printing Office, Washington (April 1965).

87) Johnson, W. A.: Nuclear Safety of Fissile Material Outside Reactors, Nucl. Safety 8(1), 16-19 (1967).

88) Paxton, H.C.: The Nature and Consequences of Nuclear Accidents. US-Report SC-DC-67-1305. Nuclear Criticality Safety Conference (ANS), Las Vegas, Nevada (December 1966).

89) Flanary, J.R., F. H. Goode, M.J. Bradley. J. W. Ulmann, L. M. Ferris, and G. C. Wall: Hot Cell Studies of Aqueous Dissolution Processes for Irradiated Carbide Reactor Fuels. US-Report ORNL-3660 (Sept. 1964).

90) Ferris, L. M., and $M$. J. Bradley: Off Gases from the Reactions of Uranium Carbides with Nitric Acid at $90^{\circ} \mathrm{C}$. Us-Report ORNL-3719 (December 1964).

91) Zust, H. E., H. R. v. Gunten u. P. Baertschi: Verfahren und Einrichtung zur Aufbereitung von in Kernreaktoren verbrauchten Spaltstoffelementen auf Carbidbasis. DBP-Auslegeschrift 1464647 (1963).

92) Stade u. Wügassen: Nucl. Eng. 12, 756 (1967).

93) Ochsenfeld, W.: Wiederaufarbeitung der Brennelemente Schneller Brutreaktoren. Atomwirtschaft 13, 422 (1968).

94) Shaw, M.: Current AEC Programms. In: Nuclear Power Fuel Processing Technology and Economics, Conf-670542.

95) Europäisches Institut für Transuranelemente Karlsruhe, Progress Report No. 6, Juli-Dezember 1968, Kap. 6.1.2.

96) Blomeke, I. O., and M.F. Todd: Uranium-235 Fission-Product Production as a Function of Thermal Neutron Flux, Irradiation Time, and Decay Time. 1. Atomic Concentrations and Gross Totals. US-Report ORNL-2127, Part I, Vol. 1 (1957).

97) Stoller, S. M., and R. B. Richards: Reactor Handbook, Vol. II, S. 147. Fuel Processing (1961).

98) Flagg, J. F.: Chemical Processing of Reactor Fuels, S. 209. New York: Academic Press 1961.

99) Burris Jr., L., and G. Dillon: Estimation of Fission Product Spectra in discharged Fuel from Fast Reactors. US-Report ANL-5742 (1957).

100) Salomon, L., and E. Lopez-Menchero: Stability of $\mathrm{HNO}_{3}$-TBP Diluent Systems: Bibliography of Data Up to June 1966. Eurochemic-Report ETR-203.

101) Rigg, T., and W. Wild: Radiation Effects in Solvent Extraction Processes. Progress in Nuclear Energy, Series III, Vol. 2, S. 320. London: Pergamon Press 1958.

102) Scadden, E. M., and N.E. Ballou: Solvent Extraction Separations of Zirconium and Niobium. Anal. Chem. 11, 1602 (1953).

103) Blake, C. A., A. T. Gresky, J. M. Schmitt, and R. G. Mansfield: Comparison of Dialkyl Phenylphosphonates with Tri-n-Butyl Phosphate in Nitrate Sys- 
tems: Extraction Properties, Stability, and Effect of Diluent on the Recovery of Uranium and Thorium from Spent Fuels. US-Report ORNL-3374 (1963).

104) Marston, A. L., D. L. West, and $R$. N. Wilhite: Selection, Cost and Performance of n-Paraffin Diluents. In: Solvent Extraction Chemistry of Metals, S. 213. London: Macmillan 1965.

105) Stieglitz, L., W. Ochsenfeld u. H. Schmieder: Der Einfluß der Radiolyse von TBP auf die Pu-Ausbeute im Purex Prozeß bei hohem Plutoniumgehalt. KFK691 (1969).

106) Burger, L. L.: The Decomposition Reactions of Tributyl Phosphate and its Diluents and their Effects on Uranium Recovery Processes. In: Progress in Nuclear Energy, Series III, Vol. 2, S. 307. London: Pergamon Press 1958.

107) Moove, $R$. H.: Investigation of Solvent Degradation Products in Recycled Uranium, Recovery Plant Solvent. US-Report HW-34502 (1955).

108) Wallace, R. $M$., and $T$. H. Siddell: Effect of Solvent Degradation on the Purex Process. US-Report DP-286 (1958).

109) Davies, W.: Solubilities of Uranyl and Iron(III) Dibutyl and Monobutyl Phosphates in TBP Solvent Extraction Solutions. US-Report ORNL-3084. (1961).

110) Naylor, A.: TBP Extraction Systems. 2. TBP and Diluent Regradation, in Reprocessing of Fuel from Present and Future Power Reactors. KjellerReport KR-126, S. 120.

111) Schlea, C.S., and A.S. Jennings: Behaviour of Actinides and Fission Products in Tri-n-butyl Phosphate and in Di-2-amyl 2 butylphosphonate Solvent Extraction Processes Using Short-residence Contactors. In: Solvent Extraction Chemistry of Metals, S. 81. London: Macmillan 1965.

112) Ochsenfeld, $W$., u. H. Schmieder: Verfahren zur vollständigen Rückextraktion von wertvollen vierwertigen Metalien, insb. Plutonium aus sauren Organophosphorverbindungen, die mit diesen vierwertigen Metallen besonders stabile Komplexe bilden. DBP-Anmeldung 1968.

113) Huggard, A.J., and B.F. Warner: Investigations to Determine the Extent of Degradation of TBP/Odorless Kerosene Solvent in the New Separation Plant Windscale. Nucl. Sci. Eng. 17, 638 (1963).

114) Lane, E. S. : Performance and Degradation of Diluents for TBP and the Cleanup of Degraded Solvents. Nucl. Sci. Eng. 17, 620 (1963).

115) Blake, C. A., W. Davis, and J.M. Schmitt: Properties of Degraded TBP-Amsco Solutions and Alternative Extractant-Diluent Systems. Nucl. Sci. Eng. 17, 626 (1963).

116) -, and J.M. Schmitt: Stability of Aromatic Diluents and Solvent Extraction Reagents in Radiochemical Processing. In: Solvent Extraction Chemistry of Metals, S. 161. London: Macmillan 1965.

117) Orth, D. A., and T.W. Olcott: Purex Process Performance Versus Solvent Exposure and Treatment. Nucl. Sci. Eng. 17, 593 (1963).

118) - Performance of $3 \%$ and $30 \%$ TBP Processes. In: Solvent Extraction Chemistry of Metals, S. 47. London: Macmillan 1965.

119) Barconcelli, F., and G. Grossi: Chemical Degradation of Aromatic Diluents Exposed to Nitric Acid Attack. In: Solvent Extraction Chemistry of MetaIs, S. 197. Londion: Macmilïan 1965.

120) Lane, E. S.: Degraded TBP-Kerosene Clean-up. UKAEA-Report AERE-M. 809 (1961).

121) Cooper, V. R., and $M . T$. Wallung $J r .:$ Aqueous Processes for Separation and Decontamination of Irradiated Fuels. Proc. 2. Intern. Conf. Peaceful Uses At. - Energy, Geneva 17, 291 (1958). 
122) Rigg, T.: The Breakdown of Tri-n-Butyl Phosphate Solvents During the Processing of Extremely Radioactive Nuclear Fuels, Part I + II. UKAEAReport IGR-R/W 203 und IGR-R/W 233 (1957).

123) Kennedy, J., J.W. A. Peckett, and $J . M$. Fletcher: The Influence of High Radiation Level on the Retention of Ruthenium in Processing Solvents. In: Solvent Extraction Chemistry of Metals, S. 187. London: Macmillan 1965.

124) Zebroski, E. L., H.W. Alter, and G.D. Collins: Plutonium Fuel Fabrication and Reprocessing for Fast Ceramic Reactors. US-Report GEAP-3876 (1962).

125) Centeno, $J$, and $R$. de Witte: Experiments in Eurochemic Extraction Evaporation Pilot Plant on First Cycle of HEU Flowsheet. Eurochemic-Report ETR191 (1967).

126) Salomon, L., E. Verbecken, and E. Lopez-Menchero: Predictions on the Behaviour of First Cycle Solvent During the Processing of Highly Irradiated Fuel. Eurochemic-Report ETR-213 (1967).

127) Eargle, J.E., C. W. Swindell, and R. I. Martens: Large Scale Processing of Highly Irradiated Plutonium by Solvent Extraction and Ion Exchange, Ind. Eng. Chem. Process Design Develop. 6, 348 (1967).

128) Allerdice, R. H.: Reprocessing of Fuel from Dounreay Fast Reactor. In: Reprocessing of Fuel from Present and Future Power Reactors. Kjeller-Report KR-126, S. 394.

129) Clark $f r_{.,}$A. T.: Performances of a 10 inch Centrifugal Contactor. US-Report DP-752 (1962).

130) Kishbanyh, A. A.: Performances for a Multi-stage Centrifugal Contactor. USReport DP-841 (1963).

131) Webster, D. S. : Hydraulic Performance of a 5 inch Centrifugal Contactor. USReport DP-370 (1962).

132) Schlea, C.S., H.E. Henry, M. R. Caverly, and W.J. Jenkins: Purex Process Performance with Short Residence Time Contactors. US-Report DP-809 (1963).

133) Whatley, $M . E$., and $W . M$. Woods: The performance of an Advanced Experimental Stacked-Clone Contactor: A High-Performance Solvent Extraction Machine with Potential for Application to Very Highly Radioactive Solutions. US-Report ORNL-3533 (April 1964).

134) Whatley, $M . W ., W . S$. Groenier, and W. M. Woods : Stacked-Clone Contactor: A High-Performance Hydroclone Solvent Extraction Device. US-Report ORNL-TM-1936 (August 7, 1967).

135) Groenier, W.S.; and $M . E$. Whatley: Stacked-Clone Contactor. In: $M . E$. Whatley, P. A. Haas, R. W. Horson, A. D. Ryon, J.C. Suddath, and C. D. Watson: Unit Operations Section Quarterly Progress Report, April-June 1966, USReport ORNL-4074 (April 1967).

136) Roth, B.: Zentrifugalextraktoren für die Wiederaufarbeitung von Kernbrennstoffen mit hohem Abbrand und Plutonium-Gehalt. KFK-862 (1969).

137) König, L. A., u. S. Zehme: Die maximal zulässige Aktivitätsabgabe über die Abluft einer kerntechnischen Anlage. KFK-340 (1965).

138) Merryman, J.R., and J.H. Pashley: Engineering Development of an Adsorption Process for the Concentration and Collection of Krypton and Xenon. USReport K-1745 (1968).

139) Albenesius, E. L.: Tritium as a Product of Fission. Phys: Rev. Letters 3, 274 (1959).

140) -, and R. S. Ondrejoin: Nuclear Fission Produces Tritium. Nucleonics 18, No. 9, $100(1960)$.

141) Sloth, E. N., D. L. Horrocks, E. J. Boyce, and $M . H$. Studier: Tritium in the Thermal Neutron Fission of Uranium-235. J. Inorg. Nucl. Chem. 24, 337 (1962). 
142) Mecham, W. J.: Studies and Evaluations: Problems of Tritium in Power Reactor Fuel Cycles. US-Report ANL-7375 (1967).

143) Dudey, N. D.: Review of Low Mass Atom Production in Fast Reactors. USReport ANL-7434 (1968).

144) Nuclear Fuel Services, Inc., Spent Fuel Processing Plant-Safety Analysis. AEC-Docket No. 50-201 (October 1962).

145) Schmidt, W. C.: Treatment of Gaseous Effluents, Symposium on the Reprocessing of Irradiated Fuels, Brussels, May 20, 1957. US-Report TID-7534, S. 371.

146) Marter, $W$. L.: Radioiodine Release Incident at the Savannah River Plant. US-Report DPSPU-63-30-26 B (May 1963).

147) Bruce, F. R.: The Behavior of Fission Products in Solvent Extraction Processes, Progress in Nuclear Energy. III. Process Chemistry, S. 134, 144; F. R. Bruce, J. M. Fletcher, and H. H. Hyman, editors. New York: McGraw-Hill 1956.

148) Culler, F. L.: Reprocessing of Reactor Fuel and Blanket Materials by Solvent Extraction, Progress in Nuclear Energy. III. Process Chemistry, S. $183 ; F . R$. Bruce, J. M. Fletcher, and H. H. Hyman, editors. New York: McGraw-Hill 1956.

149) Browning Jr., W. E.: Removal of Fission Product Activity from Gases, Nucl. Safety 1(3), 42 (1960).

150) Cowser, K. E.: Current Practices in the Release and Monitoring of $I^{131}$ at NRTS, Hanford, Savannah River and ORNL. US-Report ORNL-NSIC-3 (August 1964).

151) Leger, B. M., B. M. Legler, S. F. Fairbourne, P. N. Kelly, and R. A-Robinson: Startup Operation of a Production Facility for Separating Ba-140 from MTR Fuel. US-Report IDO-14414, S. 70 (September 1957).

152) Paige, D. M., P. N. Kelly, and E. S. Grimmett: Two Gas Cleaning Problems at the Idaho Chemical Processing Plant Site. In: Fifth AEC Air Cleaning Conference, June 24-27, 1957, TID-7551, S. 17 (April 1958); Nucl. Safety 1(3), 41 (March 1960).

153) Cederberg, G. K., and D. K. MacQueen: Containment of Iodine-131 Released by the Rala Process. US-Report IDO-14566 (October 1961).

154) Bower, J.R., and G.K. Cederberg: Removal of Iodine from Ba 40 Process Off-Gas. In: ICPP Technical Progress Report for July-Sept. 1958. US-Report IDO-14457, S. 71. (W. E. Browning, Nucl. Safety 1 (3), 43 (March 1960).

155) Schmidt, W. C.: Treatment of Gaseous Effluents, Symposium on the Reprocessing of Irradiated Fuels, Brussels, May 20, 1957. US-Report TID-7534, S. 371.

156) Blasewitz, A. G., and $W . C$. Schmidt: Treatment of Radioactive Waste Gases, P/1397. Proc. 2. Intern. Conf. Peaceful Uses At. Energy, Geneva 18, 184 (1958).

157) Michels, L. R.: Désign and Operating Considerations for Off-Gas Systems in Nuclear Processing Plants. Chem. Eng. Symp. Ser., No. 28, 56, 12 (1910).

158) Keilholtz, G. W., and C.J. Barton: Behavior of Iodine in Reactor Containment System. US-Report ORNL-NSIC-4, S. 64 (February 1965).

159) Browming $J r ., W . E .:$ Removal of Radioiodine from Reactor Gases. Nucl. Safety 2 (3), 35 (1960/61).

160) - Removal of Radioiodine from Gases. Nucl. Safety 4 (2), 83 (1963).

161) - Removal of Radioiodine from Gases. Nucl. Safety 6 (3), 272 (1964/65).

162) $M c$ Cormack, $J . D$.: Some Observations on Iodine Removal from Plant Streams with Charcoal. Presented at Eighth AEC Air Cleaning Conference October 2225, 1963, Oak Ridge, Tennessee. US-Report TID-7677, S. 38. 
163) Jacobsen, W. R., and L. Jolly: Measurement of Radioiodine in Purex Stack Gases. US-Report DPSPU-63-30-4 B (May 1963).

164) McHenry, R. E.: Removal of Radioiodine from Hot-Off-Gas System by Charcoal Absorption. Isotopes Development Center Progress Report December 1962 - January 1963. US-Report ORNL-TM-532, S. 27.

165) Chamberlain, A.C., A.E.J.Eggleton, W.J.Megaw, and J.B. Morris: Physical Chemistry of Iodine and Removal of Iodine from Gas Streams. Nucl. Energy, Parts A/B 17, 519 (1963).

166) Kovach, J. L.: Evaluation of the Ignition Temperature of Activated Charcoals in Dry Air. Nucl. Safety 8 (1), 41 (1966).

167) Adams, R. E., and W.E. Browning: Estimate of the Probability and Consequences of Ignition of the HRT Charcoal Beds. US-Report CF-58-6-6, S. 8 (June 1958).

168),$- W$. E. Brotening Jr., Wm. B. Cottrell, and G. W. Parker: The Release and Absorption of Methyl Iodide in the HFIR Maximum Credible Accident. USReport ORNL-TM-1291, S. 24 (October 1, 1965).

169) Ackley, R. C., R. E. Adams, W. E. Browning Jr., G. E. Creek, and G. W. Parker : Retention of Methyl Iodide by Charcoal under Accident Conditions. In : Nuclear Safety Program Semi-Annual Progress Report for Period ending Dec. 31, 1965. US-Report ORNL-3915, S. 61.

170) Adams, R. E., R. C. Ackley, and W.E. Browning Jr.: Removal of Radioactive Methyl Iodide from Steam Air Systems. US-Report ORNL-4040 (January 1967).

171) Colline, D. A., L.R. Taylor, and R. Taylor: The Development of Impregnated Charcoals for Trapping Methyl Iodide at High Humidity, presented at Ninth AEC Air Cleaning Conference, Boston, Mass., Sept. 13-16, 1966. US-Report CONF-660904, S. 159.

172) Scharmann, A.: Radionuklidbatterien in Energiedirektumwandlung. München: K. J. Euler 1967.

173) Seaborg, G. T.: Mass Production and Practical Applications of Actinide Elements. Isotopes Radiation Technol. 6, 1 (1968).

174) Industrial Applications for Isotopic Power Generators. Intern. Symposium AERE, Harwell 1967 (ENEA).

175) Born, H. J.: Aktiniden für Radionuklidbatterien. Atompraxis 15, 32 (1969).

176) Roberts, F. P., and H. H. Van Tuyl: Promethium-146, Fission Product, and Transuranium Isotope Content of Power Reactor Fuels. US-Report BNWL-45 (1965).

177) Vondy, D. R., J. A. Lane, and A. T. Gresky: Production of $\mathrm{Np}^{237}$ and $\mathrm{Pu}^{238}$ in Thermal Power Reactors. Ind. Eng. Chem. Process Design Develop. 3, 293 (1964).

178) Deonigi, D. E., and E. A. Eschbach: Production and Indifference Pricing of Transuranium Isotopes. US-Report BNWL-223 (1966).

179) $B \ddot{a} h r, W .:$ Extraktionsverhalten von Neptunium bei der Wiederaufarbeitung von bestrahlten Kernbrennstoffen nach dem Purex-Prozeß. KFK-797 (1969).

180) Isaacson, R. E., and B. F. Judson: Ind. Eng. Chem. Process Design Develop. 3, 296 (1964).

181) Poe, W. L., A.W. Joyce, and J.R. Martens: $\mathrm{Np}^{237}$ and $\mathrm{Pu}^{238}$ Separation of Savannah River Plant. Ind. Eng. Chem. Process Design Develop. 3, 314 (1964).

182) Savannah River Laboratory, Large Scale Production and Applications of Radioisotopes. US-Report DP-1066, Vol. II, 1966.

183) Ferguson, D. E.: ORNL Transuranium Program. The production of Transuranium Elements. Nucl. Sci. Eng. 17, 435 (1963). 
184) - Transuranium-Element Processing. Isotopes Radiation Technol. 4 (4), 321 (1967).

185) Ryan, V.A., and $J . W$. Pringle: Preparation of Pure Americium. US-Report RFP-130 (1960).

186) Kingsley, R. S.: A Multi-Column Ion Exchange Purification-Concentration Process for Americium. US-Report RL-SEP-729 REV (1965).

187) Koch, G., u. J. Schön: Isolierung von Americium-241 aus Plutonium-Schrott durch Extraktion mit Tricaprylmethylammoniumnitrat. KFK-783 (1968).

188) Wheelwright, E. J., F. B. Roberts, L. A. Bray, G. L. Ritter, and L. A. Bolt: Simultaneous Recovery and Purification of $\mathrm{Pm}, \mathrm{Am}$, and $\mathrm{Cm}$ by the Use of Alternating DTPA and NTA Cation-Exchange Flowsheets. US-Report BNWLSA-1492 (1968).

189) Höhlein, B. G., H. J. Born u. W. Weintänder: Die Isolierung von Cm-242 aus neutronenbestrahltem Am-241. Radiochim. Acta. 10, 85 (1969).

190) Milller, $W .:$ Die Gewinnung von Transplutoniumelementen aus bestrahltem Americium-241. Atompraxis 75, 35 (1969).

191) Eubanks, J. D., and G. A. Burney: Curium Process Development, I. General Purpose Description. US-Report DP-1009 (1966).

192) Henry, H. E.: Isolation of Americium and Curium from $\mathrm{Al}\left(\mathrm{NO}_{3}\right)_{3}-\mathrm{NaNO}_{3}-$ $\mathrm{HNO}_{3}$ Solutions by Batch Extraction with Tributyl Phosphate. US-Report DP-972 (1965).

193) Moove, F. L.: Improved Extraction Method for Isolation of Trivalent ActinideLanthanide Elements from Nitrate Solutions. Anal. Chem. 38, 510 (1966).

194) Ooyen, J. van: Quarternary Ammonium Nitrates as Extractants for Trivalent Actinides. In: Solvent Extraction Chemistry, S. 485. Amsterdam: NorthHolland Publ. Co. 1967.

195) Honwitz, E. P., C. A. A. Bloomquist, L. J. Sauro, and D. J. Henderson: The Liquid-Liquid-Extraction of Certain Tripositive Transplutonium Ions from Salted Nitrate Solutions with a Tertiary and Quaternary Amine, J. Inorg. Nucl. Chem. 28, 2312 (1966).

196) Peppard, D. F., G.W. Mason, W.J. Driscoll, and R. J. Sironen : Acidic Esters of Orthophosphoric Acid as Selective Extractants for Metallic Cations Tracer Studies. J. Inorg. Nucl. Chem. 7, 276 (1958).

197) - - , and S. McCarty: Application of Phosphoric Acid Esters to the Isolation of Certain Trans-Plutonides by Liquid-Liquid Extraction. J. Inorg. Nucl. Chem. 12, 141 (1959).

198) Baes $J r ., C$. F.: The Extraction of Metallic Species by Dialkylphosphoric Acids. J. Inorg. Nucl. Chem. 24, 707 (1962).

199) Leuze, R. E., R. D. Baybarz, and B. Weaver: Application of Amine and Phosphonate Extractants to Transplutonium Element Production. Nucl. Sci. Eng. 17, 252 (1963).

200) Baybarz, R. D., B. S. Weaver, and H. B. Kinser: Isolation of Transplutonium Elements by Tertiary Amine Extraction. Nucl. Sci. Eng. 17, 457 (1963).

201) Leuze, R. D., R. D. Baybarz, F. A. Kappelmann, and B. Weaver: Behaviour of the Transplutonium Elements in Solvent Extraction Systems. In: SolventExtraction Chemistry of Metals, S. 423. London: Macmillan 1965.

202) Moore, F. L.: New Approach to Separation of Trivalent Actinide Elements from Lanthanide Elements - Selective Liquid-Liquid Extraction with Tricaprylmethylammonium Thiocyanate. Anal. Chem. 36, 2158 (1964).

203) Gerontopulos, P. Th., L. Rigali, and P. G. Barbano: Separation of Americium(III) from Lanthanides by Quaternary Ammonium Salt Extraction. Radiochim. Acta 4, 75 (1965). 
204) Lloyd, $M$. H., and R. E. Leuze: Anion Exchange Separation of Trivalent Actinides and Lanthanides. Nucl. Sci. Eng. 11, 274 (1961).

205) - An Anion Exchange Process for Americium-Curium Recovery from Plutonium Process Waste. Nucl. Sci. Eng. 17, 452 (1963).

206) Coleman, J. S., R. A. Penneman, T. K. Keenan, L. E. Lamar, D. E. Armstrong, and L. B. Asprey: An Anion-Exchanger Process for Gram-Scale Separation of Americium from Rare Earth. J. Inorg. Nucl. Chem. 3, 327 (1957).

207) Keenan, T. J.: Rapid and Efficient Purification of Americium. J. Inorg. Nucl. Chem. 20, 185 (1961).

208) Higgins, G. H., and W.W.T. Crane: Proc. 2. Intern. Conf. Peaceful Uses At. Energy, Geneva 17, 245 (1958).

209) Weaver, B., and $F$. A. Kappelmann: TALSPEAK: A New Method of Separat ing Americium and Curium from the Lanthanides by Extraction from an Aqueous Solution of an Aminopolyacetic Acid Complex with a Monoacidic Organophosphate or Phosphonate. US-Report ORNL-3559 (1964).

210) - - Preferential Extraction of Lanthanides over Trivalent Actinides by Monoacidic Organophosphates from Carboxylic Acids and from Mixtures of Carboxylic and Aminopolyacetic Acids. J. Inorg. Nucl. Chem. 30, 263 (1968).

211) Koch, G.: A Study of Americium-Curium Recovery from Fuel Processing High Level Waste Solutions. In: Solvent Extraction Chemistry, im Druck Proc. V. Intern. Conf. Solvent Extraction Chemistry, 1968. New York: John Wiley 1969.

212) Adar, S., R. K. Sjomblom, R. F. Barnes, P. R. Fields, E. K. Hulet, and H. D. Wilson: Ion-Exchange Behaviour of the Transuranium Elements in $\mathrm{LiNO}_{3}$ Solutions. J. Inorg. Nucl. Chem. 25, 447 (1963).

213) Marcus, Y., M. Givon, and G. R. Choppin: Anion Exchange of Metal Complexes XII - The Actinide(III)-Nitrate System. J. Inorg. Nucl. Chem. 25, 1457 (1963).

214) Horwitz, E. P., K. A. Orlandini, and C.A.A. Bloomquist: The Separation of Americium and Curium by Extraction Chromatography Using a High Molecular Weight Quaternary Ammonium Nitrate. Inorg. Nucl. Chem. Letters 2, 87 (1966).

215) Bockkarev, V.A., et E. N. Voevodin: Séparation de l'Americium et du Curium par la Méthode d'Échange Anionique avec Utilisation des Solutions concentant une Mélange d'Alcohol Methylique et d'Acide Azotique comme Eluant. Radiokhimija 7, 461 (1965); Frz. Übersetzung: Radiochimie 7, 485 (1965).

216) Burney, G. A.: Nucl. Appl. 4, 217 (1968).

Eingegangen am 1. April 1969 